\title{
Density Functional Theory Study of the Mechanism and Origins of Stereoselectivity in the Asymmetric Simmons-Smith Cyclopropanation with Charette Chiral Dioxaborolane Ligand
}

\author{
Tao Wang, Yong Liang, and Zhi-Xiang Yu* \\ Beijing National Laboratory for Molecular Sciences (BNLMS), Key Laboratory \\ of Bioorganic Chemistry and Molecular Engineering of Ministry of Education, \\ College of Chemistry, Peking University, Beijing 100871, China \\ E-mail: yuzx@pku.edu.cn
}

\section{Content}

1. Coordinates of All Stationary Points.......................................................................... S2

2. Computed Energies of All Stationary Points ................................................................ S51

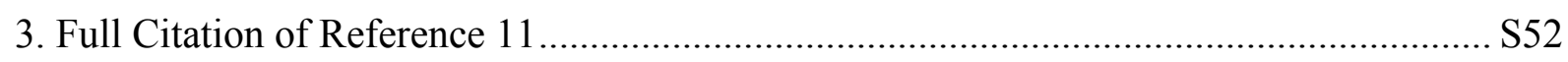

4. Comparison of Different Basis Sets and Atomic Radii Sets........................................ S53

5. DFT-Computed Free Energy Surfaces for Reactions A-D .......................................... S56 


\section{Coordinates of All Stationary Points}

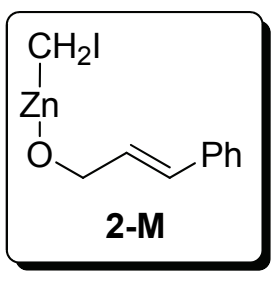

Standard orientation:

\begin{tabular}{|c|c|c|c|c|c|}
\hline \multirow{2}{*}{$\begin{array}{l}\text { Center } \\
\text { Number }\end{array}$} & \multirow{2}{*}{$\begin{array}{l}\text { Atomic } \\
\text { Number }\end{array}$} & \multirow{2}{*}{$\begin{array}{l}\text { Atomic } \\
\text { Type }\end{array}$} & \multicolumn{2}{|c|}{ Coordinates } & (Angstroms) \\
\hline & & & $X$ & $\mathrm{Y}$ & Z \\
\hline 1 & 30 & 0 & -0.736185 & 1. 004271 & -0.164624 \\
\hline 2 & 8 & 0 & -0.312503 & 2.713624 & 0.213053 \\
\hline 3 & 6 & 0 & 1. 058678 & 3.061241 & 0.162481 \\
\hline 4 & 6 & 0 & 1. 940647 & 1.893993 & 0.523436 \\
\hline 5 & 6 & 0 & 2. 743870 & 1. 259594 & -0.351735 \\
\hline 6 & 6 & 0 & -1.415304 & -0.776680 & -0.538529 \\
\hline 7 & 53 & 0 & -3.554887 & -0.879569 & 0.066012 \\
\hline 8 & 6 & 0 & 3.587139 & 0.077406 & -0.118719 \\
\hline 9 & 6 & 0 & 4. 269620 & -0.492517 & -1.208297 \\
\hline 10 & 6 & 0 & 5. 074196 & -1.619837 & -1.047726 \\
\hline 11 & 6 & 0 & 5. 216432 & -2.202473 & 0.212234 \\
\hline 12 & 6 & 0 & 4. 550063 & -1.644757 & 1. 308292 \\
\hline 13 & 6 & 0 & 3. 747629 & -0.519113 & 1. 146651 \\
\hline 14 & 1 & 0 & 1. 339517 & 3. 435781 & -0.837019 \\
\hline 15 & 1 & 0 & 1. 226519 & 3.888173 & 0.870660 \\
\hline 16 & 1 & 0 & 1.851410 & 1.545582 & 1.553471 \\
\hline 17 & 1 & 0 & 2. 791395 & 1.658122 & -1.367146 \\
\hline 18 & 1 & 0 & -1.420791 & -1.048691 & -1.593887 \\
\hline 19 & 1 & 0 & -0.935045 & -1.572253 & 0.030912 \\
\hline 20 & 1 & 0 & 4. 162538 & -0.043007 & -2.193030 \\
\hline 21 & 1 & 0 & 5.589670 & -2.041819 & -1.906283 \\
\hline 22 & 1 & 0 & 5. 843168 & -3.080332 & 0.342595 \\
\hline 23 & 1 & 0 & 4. 660773 & -2.088073 & 2.294363 \\
\hline 24 & 1 & 0 & 3.247615 & -0.094593 & 2.012470 \\
\hline
\end{tabular}




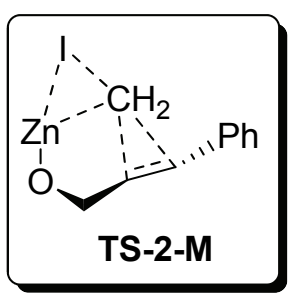

Imaginary frequency: $-277.6 \mathrm{~cm}^{-1}$

Standard orientation:

\begin{tabular}{|c|c|c|c|c|c|}
\hline \multirow{2}{*}{$\begin{array}{l}\text { Center } \\
\text { Number }\end{array}$} & \multirow{2}{*}{$\begin{array}{l}\text { Atomic } \\
\text { Number }\end{array}$} & \multirow{2}{*}{$\begin{array}{l}\text { Atomic } \\
\text { Type }\end{array}$} & \multicolumn{2}{|c|}{ Coordinates } & \multirow{2}{*}{$\begin{array}{r}(\text { Angstroms }) \\
Z\end{array}$} \\
\hline & & & X & Y & \\
\hline 1 & 30 & 0 & -1.467498 & 1.078383 & 0.115809 \\
\hline 2 & 8 & 0 & -0.943282 & 2. 834309 & 0.259111 \\
\hline 3 & 6 & 0 & 0.370965 & 2.912354 & -0.251605 \\
\hline 4 & 6 & 0 & 1. 303903 & 1. 795143 & 0.185723 \\
\hline 5 & 6 & 0 & 2.161005 & 1. 124514 & -0.627531 \\
\hline 6 & 6 & 0 & -0.161513 & -0.340045 & -0.289373 \\
\hline 7 & 53 & 0 & -2.864797 & -1.215278 & -0.010936 \\
\hline 8 & 6 & 0 & 3. 222753 & 0.183847 & -0.243827 \\
\hline 9 & 6 & 0 & 3.938123 & -0.478848 & -1.258021 \\
\hline 10 & 6 & 0 & 4. 952276 & -1.384511 & -0.951503 \\
\hline 11 & 6 & 0 & 5.276242 & -1.646249 & 0.380629 \\
\hline 12 & 6 & 0 & 4. 580739 & -0.989693 & 1. 401193 \\
\hline 13 & 6 & 0 & 3.569782 & -0.083611 & 1. 094866 \\
\hline 14 & 1 & 0 & 0.391467 & 2.979616 & -1.355282 \\
\hline 15 & 1 & 0 & 0.819813 & 3.851820 & 0.118992 \\
\hline 16 & 1 & 0 & 1.332215 & 1. 629672 & 1. 263274 \\
\hline 17 & 1 & 0 & 2. 096784 & 1. 332089 & -1.696924 \\
\hline 18 & 1 & 0 & 0.044147 & -0.697833 & -1.303241 \\
\hline 19 & 1 & 0 & 0.304191 & -1.000735 & 0.446809 \\
\hline 20 & 1 & 0 & 3.689282 & -0.280009 & -2.298054 \\
\hline 21 & 1 & 0 & 5. 489033 & -1.884831 & -1.752872 \\
\hline 22 & 1 & 0 & 6.066750 & -2.350580 & 0.623916 \\
\hline 23 & 1 & 0 & 4. 833222 & -1.180655 & 2. 440722 \\
\hline 24 & 1 & 0 & 3. 052882 & 0.427798 & 1.901772 \\
\hline
\end{tabular}




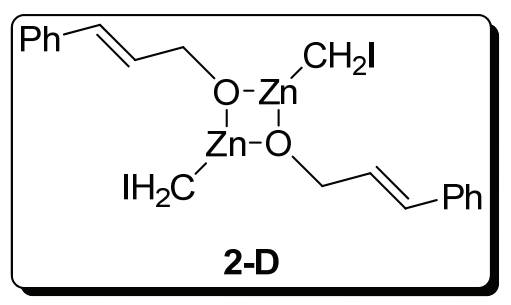

Standard orientation:

\begin{tabular}{|c|c|c|c|c|c|}
\hline \multirow{2}{*}{$\begin{array}{l}\text { Center } \\
\text { Number }\end{array}$} & \multirow{2}{*}{$\begin{array}{l}\text { Atomic } \\
\text { Number }\end{array}$} & \multirow{2}{*}{$\begin{array}{l}\text { Atomic } \\
\text { Type }\end{array}$} & \multicolumn{2}{|c|}{ Coordinates } & \multirow{2}{*}{ (Angstroms) } \\
\hline & & & $X$ & $\mathrm{Y}$ & \\
\hline 1 & 30 & 0 & -1.242843 & 0.589794 & -0.578248 \\
\hline 2 & 8 & 0 & -0.707871 & -0.863223 & 0.602855 \\
\hline 3 & 6 & 0 & -1.499391 & -1.948929 & 1.079155 \\
\hline 4 & 6 & 0 & -2.958549 & -1.607490 & 1. 012081 \\
\hline 5 & 6 & 0 & -3.847015 & -2.280747 & 0.262526 \\
\hline 6 & 6 & 0 & -2.805234 & 1. 535929 & -1.301929 \\
\hline 7 & 53 & 0 & -3.429889 & 3.055115 & 0.224591 \\
\hline 8 & 6 & 0 & -5.284184 & -2.010247 & 0.107848 \\
\hline 9 & 6 & 0 & -6.079477 & -2.945436 & -0.577958 \\
\hline 10 & 6 & 0 & -7.447120 & -2.738793 & -0.753618 \\
\hline 11 & 6 & 0 & -8.048835 & -1.584152 & -0.251244 \\
\hline 12 & 6 & 0 & -7.270562 & -0.639632 & 0.425665 \\
\hline 13 & 6 & 0 & -5.905802 & -0.848160 & 0.604094 \\
\hline 14 & 1 & 0 & -1.297958 & -2.855808 & 0.489489 \\
\hline 15 & 1 & 0 & -1.207097 & -2.163319 & 2. 118772 \\
\hline 16 & 1 & 0 & -3.266697 & -0.751327 & 1. 611742 \\
\hline 17 & 1 & 0 & -3.483458 & -3.143430 & -0.298731 \\
\hline 18 & 1 & 0 & -2.639623 & 2. 133998 & -2.198007 \\
\hline 19 & 1 & 0 & -3.701362 & 0.930036 & -1.433710 \\
\hline 20 & 1 & 0 & -5.615141 & -3.846309 & -0.973043 \\
\hline 21 & 1 & 0 & -8.041006 & -3.477971 & -1.284598 \\
\hline 22 & 1 & 0 & -9.113536 & -1.416928 & -0.389325 \\
\hline 23 & 1 & 0 & -7.728455 & 0.267184 & 0.811453 \\
\hline 24 & 1 & 0 & -5.317857 & -0.094987 & 1. 120399 \\
\hline 25 & 30 & 0 & 1. 242842 & -0.591023 & 0.578408 \\
\hline 26 & 8 & 0 & 0.707881 & 0.862035 & -0.602774 \\
\hline 27 & 6 & 0 & 1. 499273 & 1.948499 & -1.077544 \\
\hline 28 & 6 & 0 & 2. 958518 & 1.607511 & -1.010134 \\
\hline 29 & 6 & 0 & 3. 846481 & 2. 280543 & -0.259788 \\
\hline 30 & 6 & 0 & 2. 805815 & -1.537147 & 1.300836 \\
\hline 31 & 53 & 0 & 3. 430241 & -3.054364 & -0.227898 \\
\hline 32 & 6 & 0 & 5.283700 & 2. 010425 & -0.104865 \\
\hline 33 & 6 & 0 & 6.078566 & 2. 945608 & 0.581442 \\
\hline
\end{tabular}




\begin{tabular}{|c|c|c|c|c|c|}
\hline 34 & 6 & 0 & 7. 446232 & 2.739304 & 0.757328 \\
\hline 35 & 6 & 0 & 8.048393 & 1.585005 & 0.254700 \\
\hline 36 & 6 & 0 & 7. 270542 & 0.640488 & -0.422696 \\
\hline 37 & 6 & 0 & 5.905759 & 0.848682 & -0.601361 \\
\hline 38 & 1 & 0 & 1. 297245 & 2.854733 & -0.487081 \\
\hline 39 & 1 & 0 & 1. 207428 & 2. 163842 & -2.117093 \\
\hline 40 & 1 & 0 & 3.267186 & 0.751902 & -1.610315 \\
\hline 41 & 1 & 0 & 3.482441 & 3. 142691 & 0.301980 \\
\hline 42 & 1 & 0 & 2.640995 & -2.136406 & 2. 196260 \\
\hline 43 & 1 & 0 & 3.701804 & -0.931076 & 1. 432736 \\
\hline 44 & 1 & 0 & 5.613881 & 3.846210 & 0.976735 \\
\hline 45 & 1 & 0 & 8. 039788 & 3.478480 & 1. 288683 \\
\hline 46 & 1 & 0 & 9.113110 & 1. 418040 & 0.392966 \\
\hline 47 & 1 & 0 & 7. 728778 & -0.266070 & -0.808681 \\
\hline 48 & 1 & 0 & 5. 318144 & 0.095513 & -1.118052 \\
\hline
\end{tabular}

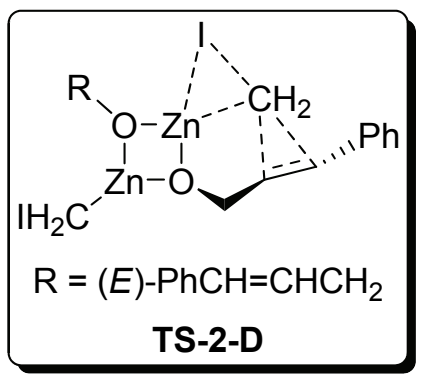

Imaginary frequency: $-316.1 \mathrm{~cm}^{-1}$

Standard orientation:

\begin{tabular}{|c|c|c|c|c|c|}
\hline \multirow{2}{*}{$\begin{array}{l}\text { Center } \\
\text { Number }\end{array}$} & \multirow{2}{*}{$\begin{array}{l}\text { Atomic } \\
\text { Number }\end{array}$} & \multirow{2}{*}{$\begin{array}{l}\text { Atomic } \\
\text { Type }\end{array}$} & \multicolumn{2}{|c|}{ Coordinates } & (Angstroms) \\
\hline & & & $X$ & Y & Z \\
\hline 1 & 30 & 0 & -1.274377 & 1. 368788 & -0.421987 \\
\hline 2 & 8 & 0 & -0.744066 & 0.019170 & -1.843454 \\
\hline 3 & 6 & 0 & -1.756236 & -0.937285 & -2.083724 \\
\hline 4 & 6 & 0 & -2.580641 & -1.273294 & -0.854138 \\
\hline 5 & 6 & 0 & -3.915271 & -1.541655 & -0.882892 \\
\hline 6 & 6 & 0 & -3.183348 & 0.927254 & -0.158355 \\
\hline 7 & 53 & 0 & -2.828823 & 3. 647418 & 0.200436 \\
\hline 8 & 6 & 0 & -4.739739 & -2.127894 & 0.175929 \\
\hline 9 & 6 & 0 & -6.128845 & -2.231528 & -0.028449 \\
\hline 10 & 6 & 0 & -6.961059 & -2.782399 & 0.943784 \\
\hline 11 & 6 & 0 & -6.421348 & -3.243208 & 2.145855 \\
\hline 12 & 6 & 0 & -5.042542 & -3.152211 & 2.363749 \\
\hline 13 & 6 & 0 & -4.210655 & -2.605379 & 1. 391893 \\
\hline
\end{tabular}




\begin{tabular}{|c|c|c|c|c|c|}
\hline 14 & 1 & 0 & -2.423342 & -0.582483 & -2.884211 \\
\hline 15 & 1 & 0 & -1.300723 & -1.874227 & -2.443171 \\
\hline 16 & 1 & 0 & -1.996475 & -1.536738 & 0.027420 \\
\hline 17 & 1 & 0 & -4.449821 & -1.309068 & -1.804998 \\
\hline 18 & 1 & 0 & -3.895148 & 1. 105973 & -0.963186 \\
\hline 19 & 1 & 0 & -3.692673 & 0.726594 & 0.784832 \\
\hline 20 & 1 & 0 & -6.554797 & -1.871426 & -0.962098 \\
\hline 21 & 1 & 0 & -8.030228 & -2.850691 & 0.763060 \\
\hline 22 & 1 & 0 & -7.066807 & -3.673557 & 2.906332 \\
\hline 23 & 1 & 0 & -4.614336 & -3.515187 & 3.294090 \\
\hline 24 & 1 & 0 & -3.141232 & -2.557482 & 1. 575194 \\
\hline 25 & 30 & 0 & 1. 093040 & -0.215153 & -1.206074 \\
\hline 26 & 8 & 0 & 0.601529 & 1. 243367 & 0.015482 \\
\hline 27 & 6 & 0 & 1. 445931 & 2. 104831 & 0.782650 \\
\hline 28 & 6 & 0 & 2. 768969 & 1. 446574 & 1. 034773 \\
\hline 29 & 6 & 0 & 3.938797 & 1. 938813 & 0.594660 \\
\hline 30 & 6 & 0 & 2.572130 & -1.467322 & -1.532569 \\
\hline 31 & 53 & 0 & 2. 279896 & -3.214211 & -0.154268 \\
\hline 32 & 6 & 0 & 5.281291 & 1. 364800 & 0.768799 \\
\hline 33 & 6 & 0 & 6. 397987 & 2. 123024 & 0.374635 \\
\hline 34 & 6 & 0 & 7. 693458 & 1. 627462 & 0.516379 \\
\hline 35 & 6 & 0 & 7. 899508 & 0.355198 & 1. 051520 \\
\hline 36 & 6 & 0 & 6. 799384 & -0.415405 & 1.441538 \\
\hline 37 & 6 & 0 & 5.506371 & 0.081344 & 1. 303076 \\
\hline 38 & 1 & 0 & 1. 590938 & 3. 059109 & 0.256294 \\
\hline 39 & 1 & 0 & 0.940267 & 2. 328308 & 1. 733153 \\
\hline 40 & 1 & 0 & 2. 729709 & 0.516651 & 1.601393 \\
\hline 41 & 1 & 0 & 3.915334 & 2. 884240 & 0.049934 \\
\hline 42 & 1 & 0 & 2. 607031 & -1.930478 & -2.518345 \\
\hline 43 & 1 & 0 & 3.564864 & -1.103747 & -1.269381 \\
\hline 44 & 1 & 0 & 6. 243039 & 3. 114829 & -0.044282 \\
\hline 45 & 1 & 0 & 8.540420 & 2. 234180 & 0.207294 \\
\hline 46 & 1 & 0 & 8.907100 & -0.036483 & 1. 160702 \\
\hline 47 & 1 & 0 & 6.950080 & -1.410312 & 1.851766 \\
\hline 48 & 1 & 0 & 4. 665490 & -0.537631 & 1. 602193 \\
\hline
\end{tabular}




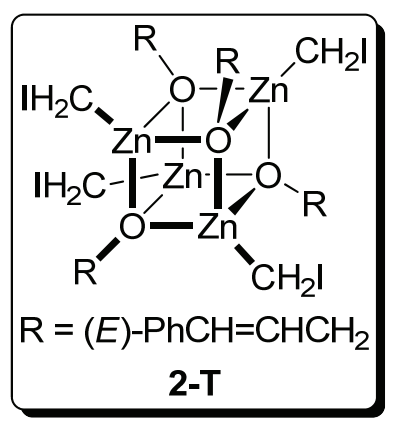

Standard orientation:

\begin{tabular}{|c|c|c|c|c|c|}
\hline & & Atomic & \multicolumn{2}{|c|}{ Coordinates } & (Angstroms) \\
\hline Number & Number & Tyре & $X$ & Y & Z \\
\hline 1 & 30 & 0 & -1.112494 & -1.091856 & -1.091362 \\
\hline 2 & 8 & 0 & 0.981597 & -0.995262 & -0.968601 \\
\hline 3 & 6 & 0 & 1. 793092 & -1.853737 & -1.810075 \\
\hline 4 & 6 & 0 & 3.251630 & -1.725791 & -1.495520 \\
\hline 5 & 6 & 0 & 3.985470 & -2.744713 & -1.016012 \\
\hline 6 & 6 & 0 & -2.169804 & -2.162389 & -2.378224 \\
\hline 7 & 53 & 0 & -1.618314 & -4.328994 & -2.237743 \\
\hline 8 & 6 & 0 & 5.421160 & -2.754894 & -0.700642 \\
\hline 9 & 6 & 0 & 5.956865 & -3.877232 & -0.042860 \\
\hline 10 & 6 & 0 & 7.311550 & -3.944324 & 0.279772 \\
\hline 11 & 6 & 0 & 8.162567 & -2.890291 & -0.056193 \\
\hline 12 & 6 & 0 & 7.646385 & -1.769330 & -0.714663 \\
\hline 13 & 6 & 0 & 6.292775 & -1.700760 & -1.033134 \\
\hline 14 & 1 & 0 & 1. 461004 & -2.889370 & -1.678585 \\
\hline 15 & 1 & 0 & 1. 598928 & -1.572743 & -2.854786 \\
\hline 16 & 1 & 0 & 3.701696 & -0.752007 & -1.689772 \\
\hline 17 & 1 & 0 & 3.473280 & -3.688443 & -0.826284 \\
\hline 18 & 1 & 0 & -3.244326 & -2.174996 & -2.191840 \\
\hline 19 & 1 & 0 & -2.004472 & -1.937974 & -3.433050 \\
\hline 20 & 1 & 0 & 5. 296896 & -4.700186 & 0.220777 \\
\hline 21 & 1 & 0 & 7.701721 & -4.820623 & 0.790238 \\
\hline 22 & 1 & 0 & 9.220423 & -2.942190 & 0.186829 \\
\hline 23 & 1 & 0 & 8.301698 & -0.946740 & -0.987312 \\
\hline 24 & 1 & 0 & 5. 914561 & -0.826565 & -1.554650 \\
\hline 25 & 30 & 0 & 1. 112678 & 1. 091961 & -1.091032 \\
\hline 26 & 8 & 0 & -0.981575 & 0.995225 & -0.968604 \\
\hline 27 & 6 & 0 & -1.793025 & 1. 853669 & -1.810206 \\
\hline 28 & 6 & 0 & -3.251548 & 1. 725870 & -1.495599 \\
\hline 29 & 6 & 0 & -3.985280 & 2.744863 & -1.016070 \\
\hline 30 & 6 & 0 & 2. 169953 & 2. 162524 & -2.377890 \\
\hline 31 & 53 & 0 & 1. 618232 & 4. 329081 & -2.237788 \\
\hline
\end{tabular}




\begin{tabular}{|c|c|c|c|c|c|}
\hline 32 & 6 & 0 & -5.420920 & 2. 755122 & -0.700509 \\
\hline 33 & 6 & 0 & -5.956390 & 3.877330 & -0.042309 \\
\hline 34 & 6 & 0 & -7.311003 & 3.944447 & 0.280616 \\
\hline 35 & 6 & 0 & -8.162169 & 2. 890572 & -0.055466 \\
\hline 36 & 6 & 0 & -7.646222 & 1. 769753 & -0.714364 \\
\hline 37 & 6 & 0 & -6.292689 & 1. 701164 & -1.033147 \\
\hline 38 & 1 & 0 & -1.598852 & 1. 572482 & -2.854863 \\
\hline 39 & 1 & 0 & -1.460826 & 2. 889284 & -1.678875 \\
\hline 40 & 1 & 0 & -3.701728 & 0.752128 & -1.689798 \\
\hline 41 & 1 & 0 & -3.473007 & 3.688562 & -0.826420 \\
\hline 42 & 1 & 0 & 2. 004691 & 1. 937836 & -3.432672 \\
\hline 43 & 1 & 0 & 3.244453 & 2. 175335 & -2.191431 \\
\hline 44 & 1 & 0 & -5.296285 & 4. 700140 & 0.221438 \\
\hline 45 & 1 & 0 & -7.701004 & 4. 820637 & 0.791399 \\
\hline 46 & 1 & 0 & -9.219972 & 2. 942482 & 0.187787 \\
\hline 47 & 1 & 0 & -8.301672 & 0.947292 & -0.987076 \\
\hline 48 & 1 & 0 & -5.914644 & 0.827102 & -1.555007 \\
\hline 49 & 30 & 0 & -1.112768 & 1. 091826 & 1. 091311 \\
\hline 50 & 8 & 0 & 0.981410 & 0.995113 & 0.968893 \\
\hline 51 & 6 & 0 & 1. 792863 & 1. 853559 & 1.810464 \\
\hline 52 & 6 & 0 & 3.251372 & 1. 725941 & 1. 495625 \\
\hline 53 & 6 & 0 & 3. 984903 & 2.745035 & 1. 016011 \\
\hline 54 & 6 & 0 & -2.170304 & 2. 162249 & 2.378067 \\
\hline 55 & 53 & 0 & -1.618548 & 4. 328811 & 2. 238095 \\
\hline 56 & 6 & 0 & 5. 420502 & 2. 755479 & 0.700247 \\
\hline 57 & 6 & 0 & 5.955735 & 3.877715 & 0.041905 \\
\hline 58 & 6 & 0 & 7. 310309 & 3.945023 & -0.281145 \\
\hline 59 & 6 & 0 & 8. 161687 & 2. 891330 & 0.054973 \\
\hline 60 & 6 & 0 & 7. 645981 & 1. 770489 & 0.714021 \\
\hline 61 & 6 & 0 & 6.292476 & 1. 701694 & 1. 032898 \\
\hline 62 & 1 & 0 & 1. 460540 & 2. 889158 & 1. 679318 \\
\hline 63 & 1 & 0 & 1. 598927 & 1. 572213 & 2. 855119 \\
\hline 64 & 1 & 0 & 3.701661 & 0.752225 & 1. 689706 \\
\hline 65 & 1 & 0 & 3.472495 & 3.688685 & 0.826477 \\
\hline 66 & 1 & 0 & -3.244761 & 2. 175000 & 2. 191323 \\
\hline 67 & 1 & 0 & -2.005338 & 1.937603 & 3.432901 \\
\hline 68 & 1 & 0 & 5. 295481 & 4.700405 & -0.221849 \\
\hline 69 & 1 & 0 & 7. 700116 & 4.821230 & -0.792048 \\
\hline 70 & 1 & 0 & 9. 219463 & 2.943407 & -0.188359 \\
\hline 71 & 1 & 0 & 8.301586 & 0.948178 & 0.986812 \\
\hline 72 & 1 & 0 & 5.914629 & 0.827622 & 1. 554887 \\
\hline 73 & 30 & 0 & 1. 112265 & -1.092100 & 1. 091495 \\
\hline 74 & 8 & 0 & -0.981660 & -0.995451 & 0.968792 \\
\hline 75 & 6 & 0 & -1.793053 & -1.854223 & 1. 810117 \\
\hline
\end{tabular}




$\begin{array}{rrrrrr}76 & 6 & 0 & -3.251573 & -1.726422 & 1.495502 \\ 77 & 6 & 0 & -3.985240 & -2.745240 & 1.015512 \\ 78 & 6 & 0 & 2.170320 & -2.162384 & 2.377936 \\ 79 & 53 & 0 & 1.618937 & -4.329048 & 2.238284 \\ 80 & 6 & 0 & -5.420882 & -2.755414 & 0.699935 \\ 81 & 6 & 0 & -5.956279 & -3.877298 & 0.041124 \\ 82 & 6 & 0 & -7.310889 & -3.944325 & -0.281835 \\ 83 & 6 & 0 & -8.162127 & -2.890696 & 0.054833 \\ 84 & 6 & 0 & -7.646253 & -1.770209 & 0.714353 \\ 85 & 6 & 0 & -6.292721 & -1.701701 & 1.033162 \\ 86 & 1 & 0 & -1.598981 & -1.573275 & 2.854857 \\ 87 & 1 & 0 & -1.460746 & -2.889774 & 1.678548 \\ 88 & 1 & 0 & -3.701808 & -0.752782 & 1.690096 \\ 89 & 1 & 0 & -3.472932 & -3.688837 & 0.825456 \\ 90 & 1 & 0 & 2.005514 & -1.937616 & 3.432776 \\ 91 & 1 & 0 & 3.244737 & -2.175003 & 2.190982 \\ 92 & 1 & 0 & -5.296123 & -4.699923 & -0.223069 \\ 93 & 1 & 0 & -7.700831 & -4.820262 & -0.793099 \\ 94 & 1 & 0 & -9.219929 & -2.942544 & -0.188437 \\ 95 & 1 & 0 & -8.301768 & -0.947959 & 0.987546 \\ 96 & 1 & 0 & -5.914735 & -0.827913 & 1.555523 \\ -----------------------------------------------------\end{array}$

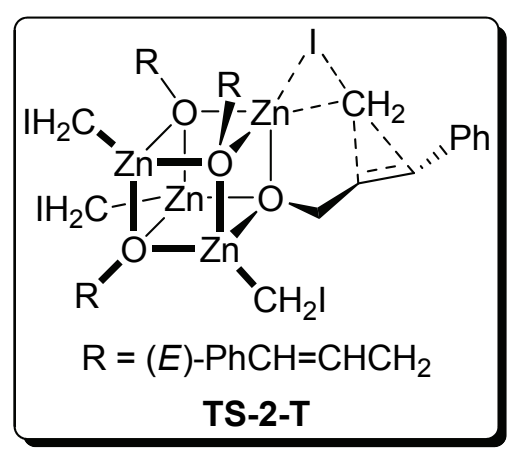

Imaginary frequency: $-349.7 \mathrm{~cm}^{-1}$

Standard orientation:

\begin{tabular}{|c|c|c|c|c|c|}
\hline Center & A tomic & A tomic & \multicolumn{2}{|c|}{ Coordinates } & (Angstroms) \\
\hline Number & Number & Туре & X & Y & Z \\
\hline 1 & 30 & 0 & -1.188743 & 0.988933 & 0.893806 \\
\hline 2 & 8 & 0 & 0.872419 & 1. 129332 & 0.826585 \\
\hline 3 & 6 & 0 & 1. 590908 & 1. 992860 & 1. 748766 \\
\hline 4 & 6 & 0 & 3. 070903 & 1. 923229 & 1.532678 \\
\hline 5 & 6 & 0 & 3.803171 & 2. 983884 & 1. 151240 \\
\hline 6 & 6 & 0 & -2.802010 & 2. 147984 & 1. 025596 \\
\hline
\end{tabular}




\begin{tabular}{|c|c|c|c|c|c|}
\hline 7 & 53 & 0 & -1.990840 & 1. 683872 & 3.637485 \\
\hline 8 & 6 & 0 & 5.257039 & 3. 055163 & 0.946853 \\
\hline 9 & 6 & 0 & 5.802851 & 4. 235613 & 0.410136 \\
\hline 10 & 6 & 0 & 7. 174350 & 4. 362597 & 0.194160 \\
\hline 11 & 6 & 0 & 8. 032271 & 3.310145 & 0.517107 \\
\hline 12 & 6 & 0 & 7. 506045 & 2. 131157 & 1. 055267 \\
\hline 13 & 6 & 0 & 6.136060 & 2.003564 & 1. 267650 \\
\hline 14 & 1 & 0 & 1. 235404 & 3. 021024 & 1. 615022 \\
\hline 15 & 1 & 0 & 1. 325373 & 1. 681281 & 2. 766313 \\
\hline 16 & 1 & 0 & 3.539496 & 0.958187 & 1. 725963 \\
\hline 17 & 1 & 0 & 3.273625 & 3. 918422 & 0.963155 \\
\hline 18 & 1 & 0 & -2.713177 & 3.230600 & 1. 122804 \\
\hline 19 & 1 & 0 & -3.801871 & 1.816643 & 1. 294335 \\
\hline 20 & 1 & 0 & 5. 137893 & 5.058402 & 0.158316 \\
\hline 21 & 1 & 0 & 7. 571882 & 5.283943 & -0.222875 \\
\hline 22 & 1 & 0 & 9. 102619 & 3. 407499 & 0.356734 \\
\hline 23 & 1 & 0 & 8.165380 & 1. 307427 & 1. 314138 \\
\hline 24 & 1 & 0 & 5. 749752 & 1. 082816 & 1. 694601 \\
\hline 25 & 30 & 0 & 1. 194556 & -0.954477 & 0.838961 \\
\hline 26 & 8 & 0 & -0.895160 & -1.027192 & 0.627307 \\
\hline 27 & 6 & 0 & -1.646408 & -2.035178 & 1.361656 \\
\hline 28 & 6 & 0 & -3.121553 & -1.790773 & 1. 289139 \\
\hline 29 & 6 & 0 & -3.971217 & -2.596659 & 0.629712 \\
\hline 30 & 6 & 0 & 2.305849 & -2.001853 & 2. 097741 \\
\hline 31 & 53 & 0 & 1.880310 & -4.191133 & 1. 901493 \\
\hline 32 & 6 & 0 & -5.433707 & -2.464743 & 0.540692 \\
\hline 33 & 6 & 0 & -6.130111 & -3.246163 & -0.399231 \\
\hline 34 & 6 & 0 & -7.515762 & -3.157030 & -0.525791 \\
\hline 35 & 6 & 0 & -8.238716 & -2.289839 & 0.295348 \\
\hline 36 & 6 & 0 & -7.562864 & -1.514955 & 1. 244055 \\
\hline 37 & 6 & 0 & -6.177661 & -1.600173 & 1. 366651 \\
\hline 38 & 1 & 0 & -1.310706 & -2.004027 & 2. 405398 \\
\hline 39 & 1 & 0 & -1.390723 & -3.018254 & 0.953212 \\
\hline 40 & 1 & 0 & -3.483723 & -0.920305 & 1. 835384 \\
\hline 41 & 1 & 0 & -3.553254 & -3.450431 & 0.094229 \\
\hline 42 & 1 & 0 & 2. 123059 & -1.811514 & 3.156137 \\
\hline 43 & 1 & 0 & 3.379537 & -1.948348 & 1. 913868 \\
\hline 44 & 1 & 0 & -5.572533 & -3.929191 & -1.035974 \\
\hline 45 & 1 & 0 & -8.030740 & -3.770012 & -1.260508 \\
\hline 46 & 1 & 0 & -9.320040 & -2.227413 & 0.208249 \\
\hline 47 & 1 & 0 & -8.118844 & -0.853285 & 1.903138 \\
\hline 48 & 1 & 0 & -5.674067 & -1.011528 & 2. 128056 \\
\hline 49 & 30 & 0 & -0.929950 & -0.997268 & -1.454295 \\
\hline 50 & 8 & 0 & 1. 140332 & -0.737799 & -1.224008 \\
\hline
\end{tabular}




\begin{tabular}{|c|c|c|c|c|c|}
\hline 51 & 6 & 0 & 2.043954 & -1.496952 & -2.067691 \\
\hline 52 & 6 & 0 & 3.473834 & -1.317763 & -1.660917 \\
\hline 53 & 6 & 0 & 4. 226034 & -2.327260 & -1.190026 \\
\hline 54 & 6 & 0 & -1.901410 & -2.108707 & -2.772781 \\
\hline 55 & 53 & 0 & -1.298037 & -4.256929 & -2.599760 \\
\hline 56 & 6 & 0 & 5.639060 & -2.288758 & -0.787021 \\
\hline 57 & 6 & 0 & 6.168349 & -3.386382 & -0.083573 \\
\hline 58 & 6 & 0 & 7. 500880 & -3.403242 & 0.325797 \\
\hline 59 & 6 & 0 & 8.336342 & -2.324725 & 0.030075 \\
\hline 60 & 6 & 0 & 7. 827223 & -1.229895 & -0.675970 \\
\hline 61 & 6 & 0 & 6. 494802 & -1.210518 & -1.079906 \\
\hline 62 & 1 & 0 & 1.763199 & -2.555126 & -2.018342 \\
\hline 63 & 1 & 0 & 1.893301 & -1.160761 & -3.103433 \\
\hline 64 & 1 & 0 & 3.886292 & -0.314896 & -1.773768 \\
\hline 65 & 1 & 0 & 3.747677 & -3.300308 & -1.074637 \\
\hline 66 & 1 & 0 & -2.982786 & -2.146161 & -2.634477 \\
\hline 67 & 1 & 0 & -1.698243 & -1.880908 & -3.820176 \\
\hline 68 & 1 & 0 & 5.520081 & -4.227762 & 0.149063 \\
\hline 69 & 1 & 0 & 7.886473 & -4.259762 & 0.872010 \\
\hline 70 & 1 & 0 & 9.377824 & -2.338459 & 0.339839 \\
\hline 71 & 1 & 0 & 8. 472138 & -0.390057 & -0.919284 \\
\hline 72 & 1 & 0 & 6.121597 & -0.358415 & -1.640248 \\
\hline 73 & 30 & 0 & 1. 102867 & 1. 354503 & -1.226509 \\
\hline 74 & 8 & 0 & -0.944293 & 1. 055527 & -1.269737 \\
\hline 75 & 6 & 0 & -1.881021 & 1.953014 & -1.862329 \\
\hline 76 & 6 & 0 & -3.240234 & 1.917066 & -1.202178 \\
\hline 77 & 6 & 0 & -4.042677 & 3.019560 & -1.129357 \\
\hline 78 & 6 & 0 & 2. 119215 & 2.668973 & -2.304269 \\
\hline 79 & 53 & 0 & 1.326417 & 4. 734210 & -1.911173 \\
\hline 80 & 6 & 0 & -5.471207 & 3.077854 & -0.829791 \\
\hline 81 & 6 & 0 & -6.094364 & 4. 339819 & -0.768697 \\
\hline 82 & 6 & 0 & -7.456037 & 4. 457140 & -0.500667 \\
\hline 83 & 6 & 0 & -8.225899 & 3.312507 & -0.284390 \\
\hline 84 & 6 & 0 & -7.623021 & 2. 051311 & -0.340280 \\
\hline 85 & 6 & 0 & -6.264066 & 1.932268 & -0.611994 \\
\hline 86 & 1 & 0 & -2.011569 & 1. 680875 & -2.921376 \\
\hline 87 & 1 & 0 & -1.480464 & 2.974242 & -1.838435 \\
\hline 88 & 1 & 0 & -3.673975 & 0.924225 & -1.091956 \\
\hline 89 & 1 & 0 & -3.574473 & 3.985205 & -1.324571 \\
\hline 90 & 1 & 0 & 2. 029377 & 2.582009 & -3.388111 \\
\hline 91 & 1 & 0 & 3.174430 & 2.768491 & -2.048887 \\
\hline 92 & 1 & 0 & -5.497518 & 5.233545 & -0.934900 \\
\hline 93 & 1 & 0 & -7.915383 & 5.440798 & -0.459216 \\
\hline 94 & 1 & 0 & -9.288385 & 3.400539 & -0.075328 \\
\hline
\end{tabular}




$\begin{array}{rrrrrr}95 & 1 & 0 & -8.211823 & 1.153753 & -0.175036 \\ 96 & 1 & 0 & -5.821597 & 0.941466 & -0.655599 \\ -\end{array}$

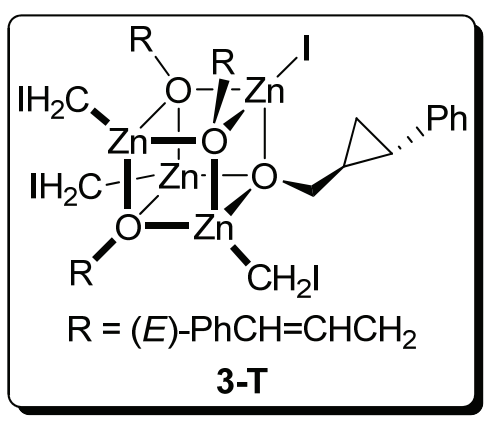

Standard orientation:

\begin{tabular}{|c|c|c|c|c|c|}
\hline \multirow{2}{*}{$\begin{array}{l}\text { Center } \\
\text { Number }\end{array}$} & \multirow{2}{*}{$\begin{array}{l}\text { Atomic } \\
\text { Number }\end{array}$} & \multirow{2}{*}{$\begin{array}{l}\text { Atomic } \\
\text { Type }\end{array}$} & \multicolumn{2}{|c|}{ Coordinates } & (Angstroms) \\
\hline & & & X & Y & Z \\
\hline 1 & 30 & 0 & -1.006386 & 0.311734 & 2. 004746 \\
\hline 2 & 8 & 0 & 0.998222 & 0.387147 & 1.583859 \\
\hline 3 & 6 & 0 & 1. 958207 & 0.817132 & 2.585080 \\
\hline 4 & 6 & 0 & 3.348265 & 0.866396 & 2. 031703 \\
\hline 5 & 6 & 0 & 4. 057510 & 2.004846 & 1.944955 \\
\hline 6 & 6 & 0 & -3.253276 & 2. 155228 & -2.386467 \\
\hline 7 & 53 & 0 & -2.094998 & 0.973131 & 4. 183735 \\
\hline 8 & 6 & 0 & 5. 434643 & 2. 177723 & 1. 461280 \\
\hline 9 & 6 & 0 & 5.937751 & 3. 483829 & 1. 319350 \\
\hline 10 & 6 & 0 & 7. 237517 & 3.709895 & 0.868416 \\
\hline 11 & 6 & 0 & 8.065565 & 2.630989 & 0.555143 \\
\hline 12 & 6 & 0 & 7. 581484 & 1. 326040 & 0.694273 \\
\hline 13 & 6 & 0 & 6.282517 & 1. 100540 & 1. 141183 \\
\hline 14 & 1 & 0 & 1. 659644 & 1.802857 & 2.958868 \\
\hline 15 & 1 & 0 & 1. 899675 & 0.110820 & 3.424381 \\
\hline 16 & 1 & 0 & 3. 772283 & -0.085966 & 1. 713553 \\
\hline 17 & 1 & 0 & 3. 569852 & 2.926366 & 2.264649 \\
\hline 18 & 1 & 0 & -3.881978 & 1. 332003 & -2.056135 \\
\hline 19 & 1 & 0 & -2.953624 & 2. 095284 & -3.429278 \\
\hline 20 & 1 & 0 & 5. 296258 & 4. 327160 & 1. 563502 \\
\hline 21 & 1 & 0 & 7. 603380 & 4. 727852 & 0.765535 \\
\hline 22 & 1 & 0 & 9.081567 & 2. 802992 & 0.210327 \\
\hline 23 & 1 & 0 & 8. 219667 & 0.478915 & 0.458931 \\
\hline 24 & 1 & 0 & 5.930485 & 0.079182 & 1. 251514 \\
\hline 25 & 30 & 0 & 1. 080766 & -1.547864 & 0.740257 \\
\hline 26 & 8 & 0 & -1.007041 & -1.474141 & 1. 008387 \\
\hline 27 & 6 & 0 & -1.756159 & -2.630382 & 1. 484757 \\
\hline
\end{tabular}




\begin{tabular}{|c|c|c|c|c|c|}
\hline 28 & 6 & 0 & -3.212919 & -2.312735 & 1. 613118 \\
\hline 29 & 6 & 0 & -4.160123 & -2.877374 & 0.844156 \\
\hline 30 & 6 & 0 & 2.301311 & -3.007648 & 1. 281362 \\
\hline 31 & 53 & 0 & 1. 641074 & -4.961782 & 0.417881 \\
\hline 32 & 6 & 0 & -5.609521 & -2.635460 & 0.886887 \\
\hline 33 & 6 & 0 & -6.422905 & -3.243722 & -0.086057 \\
\hline 34 & 6 & 0 & -7.802078 & -3.038240 & -0.102824 \\
\hline 35 & 6 & 0 & -8.397833 & -2.221193 & 0.858830 \\
\hline 36 & 6 & 0 & -7.603478 & -1.613861 & 1. 837202 \\
\hline 37 & 6 & 0 & -6.226992 & -1.818266 & 1.853504 \\
\hline 38 & 1 & 0 & -1.332839 & -2.919756 & 2. 455331 \\
\hline 39 & 1 & 0 & -1.592337 & -3.455515 & 0.784321 \\
\hline 40 & 1 & 0 & -3.474563 & -1.587744 & 2.383013 \\
\hline 41 & 1 & 0 & -3.838764 & -3.596912 & 0.089978 \\
\hline 42 & 1 & 0 & 2. 340419 & -3.212915 & 2.352220 \\
\hline 43 & 1 & 0 & 3.321895 & -2.908727 & 0.910117 \\
\hline 44 & 1 & 0 & -5.962781 & -3.882237 & -0.836622 \\
\hline 45 & 1 & 0 & -8.409828 & -3.517708 & -0.865428 \\
\hline 46 & 1 & 0 & -9.472440 & -2.060531 & 0.851108 \\
\hline 47 & 1 & 0 & -8.060945 & -0.981316 & 2. 593254 \\
\hline 48 & 1 & 0 & -5.629249 & -1.345092 & 2.627115 \\
\hline 49 & 30 & 0 & -1.460268 & -0.666745 & -0.865118 \\
\hline 50 & 8 & 0 & 0.635862 & -0.521658 & -1.003270 \\
\hline 51 & 6 & 0 & 1. 291022 & -0.890393 & -2.246154 \\
\hline 52 & 6 & 0 & 2.781072 & -0.917271 & -2.102196 \\
\hline 53 & 6 & 0 & 3. 499280 & -2.048715 & -2.207851 \\
\hline 54 & 6 & 0 & -2.661876 & -1.318958 & -2.295766 \\
\hline 55 & 53 & 0 & -2.105705 & -3.399337 & -2.910561 \\
\hline 56 & 6 & 0 & 4. 958229 & -2.200835 & -2.112548 \\
\hline 57 & 6 & 0 & 5. 494012 & -3.494859 & -1.978593 \\
\hline 58 & 6 & 0 & 6.869999 & -3.696306 & -1.879624 \\
\hline 59 & 6 & 0 & 7. 740810 & -2.606290 & -1.924459 \\
\hline 60 & 6 & 0 & 7. 223994 & -1.314441 & -2.067286 \\
\hline 61 & 6 & 0 & 5.849372 & -1.112381 & -2.159704 \\
\hline 62 & 1 & 0 & 0.919095 & -1.872568 & -2.557456 \\
\hline 63 & 1 & 0 & 0.988117 & -0.160671 & -3.010010 \\
\hline 64 & 1 & 0 & 3.267223 & 0.039069 & -1.908066 \\
\hline 65 & 1 & 0 & 2.954326 & -2.978626 & -2.374014 \\
\hline 66 & 1 & 0 & -3.707956 & -1.421811 & -2.005098 \\
\hline 67 & 1 & 0 & -2.616253 & -0.767624 & -3.235118 \\
\hline 68 & 1 & 0 & 4. 818572 & -4.346177 & -1.943240 \\
\hline 69 & 1 & 0 & 7. 261013 & -4.704337 & -1.772116 \\
\hline 70 & 1 & 0 & 8.814310 & -2.760634 & -1.857349 \\
\hline 71 & 1 & 0 & 7. 894576 & -0.460895 & -2.115130 \\
\hline
\end{tabular}




$\begin{array}{rrrrrr}72 & 1 & 0 & 5.467264 & -0.103761 & -2.286831 \\ 73 & 30 & 0 & 0.795695 & 1.402372 & -0.223256 \\ 74 & 8 & 0 & -1.270652 & 1.159305 & 0.147059 \\ 75 & 6 & 0 & -2.200903 & 2.261494 & 0.015960 \\ 76 & 6 & 0 & -2.298023 & 2.770975 & -1.398163 \\ 77 & 6 & -3.550023 & 3.528140 & -1.817974 \\ 78 & 6 & 0 & 1.712611 & 3.014067 & -0.912673 \\ 79 & 53 & 0 & 1.234334 & 4.767584 & 0.398126 \\ 80 & 6 & 0 & -3.482007 & 4.771238 & -2.641525 \\ 81 & 6 & 0 & -4.360382 & 5.828117 & -2.361699 \\ 82 & 6 & 0 & -4.329570 & 7.007714 & -3.106438 \\ 83 & 6 & 0 & -3.415347 & 7.154383 & -4.150268 \\ 84 & 6 & 0 & -2.534930 & 6.109759 & -4.440633 \\ 85 & 6 & 0 & -2.568636 & 4.931807 & -3.695292 \\ 86 & 1 & 0 & -1.867976 & 3.065835 & 0.682344 \\ 87 & 1 & 0 & -3.187209 & 1.926600 & 0.363039 \\ 88 & 1 & 0 & -1.365869 & 3.169566 & -1.795891 \\ 89 & 1 & 0 & -4.340418 & 3.550488 & -1.069817 \\ 90 & 1 & 0 & 1.416457 & 3.361553 & -1.903400 \\ 91 & 1 & 0 & 2.801586 & 2.969337 & -0.870799 \\ 92 & 1 & 0 & -5.076004 & 5.723550 & -1.549296 \\ 93 & 1 & 0 & -5.020805 & 7.811933 & -2.868352 \\ 94 & 1 & -3.387973 & 8.071772 & -4.731716 \\ 95 & 1 & -1.818009 & 6.211349 & -5.251259 \\ 96 & 1 & -1.873697 & 4.132410 & -3.942176 \\ -------------------------------\end{array}$

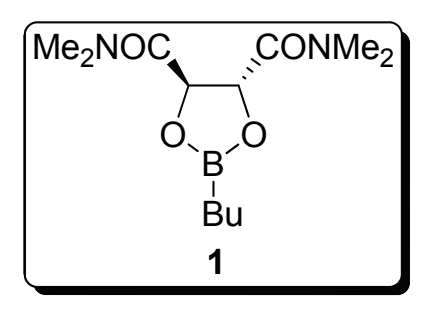

Standard orientation:

\begin{tabular}{cccrrr} 
Center & Atomic & Atomic & \multicolumn{2}{c}{ Coordinates } & (Angstroms) \\
Number & Number & Type & X & Y \\
---1 & 0 & -0.570694 & 0.413392 & 0.514722 \\
1 & 6 & 0 & -1.011552 & -0.397671 & -0.735845 \\
2 & 6 & 0 & 0.175166 & -1.141580 & -1.078715 \\
3 & 8 & 0 & 1.252285 & -0.487949 & -0.511882 \\
4 & 5 & 0 & 0.858447 & 0.516868 & 0.349530 \\
5 & 8 & 0 & -1.356329 & 1.715323 & 0.748358
\end{tabular}




\begin{tabular}{|c|c|c|c|c|c|}
\hline 7 & 8 & 0 & -2.232933 & 1.659665 & 1. 608001 \\
\hline 8 & 7 & 0 & -1.113001 & 2. 841408 & 0.011523 \\
\hline 9 & 6 & 0 & -0.190453 & 2. 962334 & -1.107525 \\
\hline 10 & 6 & 0 & -1.966594 & 4. 003145 & 0.234903 \\
\hline 11 & 6 & 0 & -2.319700 & -1.189350 & -0.565353 \\
\hline 12 & 8 & 0 & -3.318507 & -0.683432 & -1.072167 \\
\hline 13 & 7 & 0 & -2.360447 & -2.359065 & 0.142090 \\
\hline 14 & 6 & 0 & -1.261708 & -2.963495 & 0.880690 \\
\hline 15 & 6 & 0 & -3.657669 & -2.997765 & 0.334590 \\
\hline 16 & 6 & 0 & 6. 255022 & -0.147151 & 0.820435 \\
\hline 17 & 6 & 0 & 5. 229963 & -0.651297 & -0.200783 \\
\hline 18 & 6 & 0 & 3.780857 & -0.332012 & 0.188887 \\
\hline 19 & 6 & 0 & 2. 747569 & -0.838308 & -0.833624 \\
\hline 20 & 1 & 0 & -0.757759 & -0.156196 & 1. 429286 \\
\hline 21 & 1 & 0 & -1.235684 & 0.273476 & -1.569909 \\
\hline 22 & 1 & 0 & 0.533296 & 2. 153089 & -1.103207 \\
\hline 23 & 1 & 0 & 0.359968 & 3.907104 & -1.020481 \\
\hline 24 & 1 & 0 & -0.729636 & 2.972619 & -2.065824 \\
\hline 25 & 1 & 0 & -2.583488 & 3.818990 & 1. 112666 \\
\hline 26 & 1 & 0 & -1.347136 & 4. 894724 & 0.391112 \\
\hline 27 & 1 & 0 & -2.616039 & 4. 177259 & -0.633662 \\
\hline 28 & 1 & 0 & -0.306420 & -2.565423 & 0.552091 \\
\hline 29 & 1 & 0 & -1.377089 & -2.804144 & 1. 962610 \\
\hline 30 & 1 & 0 & -1.256579 & -4.045018 & 0.698044 \\
\hline 31 & 1 & 0 & -3.965640 & -2.935686 & 1. 387122 \\
\hline 32 & 1 & 0 & -4.392705 & -2.486888 & -0.284816 \\
\hline 33 & 1 & 0 & -3.597229 & -4.055558 & 0.050939 \\
\hline 34 & 1 & 0 & 7. 278945 & -0.389389 & 0.513482 \\
\hline 35 & 1 & 0 & 6.087194 & -0.598339 & 1.806282 \\
\hline 36 & 1 & 0 & 6. 191834 & 0.941438 & 0.941188 \\
\hline 37 & 1 & 0 & 5.443879 & -0.210112 & -1.185123 \\
\hline 38 & 1 & 0 & 5. 340190 & -1.738174 & -0.326191 \\
\hline 39 & 1 & 0 & 3.567474 & -0.768787 & 1. 174842 \\
\hline 40 & 1 & 0 & 3. 668071 & 0.753256 & 0.316121 \\
\hline 41 & 1 & 0 & 2. 981069 & -0.423125 & -1.828281 \\
\hline 42 & 1 & 0 & 2. 836537 & -1.926816 & -0.964091 \\
\hline
\end{tabular}




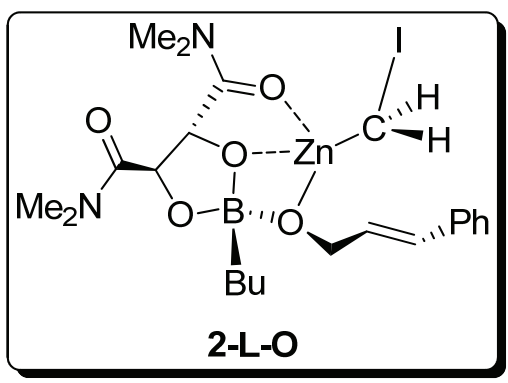

Standard orientation:

\begin{tabular}{|c|c|c|c|c|c|}
\hline \multirow{3}{*}{$\begin{array}{c}\text { Center } \\
\text { Number } \\
------- \\
1\end{array}$} & \multirow{3}{*}{$\begin{array}{c}\text { Atomic } \\
\text { Number } \\
------- \\
6\end{array}$} & \multirow{3}{*}{$\begin{array}{c}\text { Atomic } \\
\text { Type } \\
0\end{array}$} & \multicolumn{2}{|c|}{ Coordinates } & \multirow{3}{*}{$\begin{array}{r}(\text { Angstroms }) \\
-0.148653\end{array}$} \\
\hline & & & $X$ & Y & \\
\hline & & & -2.275891 & 1. 056824 & \\
\hline 2 & 6 & 0 & -2.821984 & -0.213437 & -0.897180 \\
\hline 3 & 8 & 0 & -2.596959 & -1.285931 & -0.019336 \\
\hline 4 & 5 & 0 & -1.500838 & -0.982540 & 0.884441 \\
\hline 5 & 8 & 0 & -1.454405 & 0.548856 & 0.889637 \\
\hline 6 & 8 & 0 & -0.135219 & -1.234728 & 0.213209 \\
\hline 7 & 30 & 0 & 0.624857 & 0.631778 & 0.422662 \\
\hline 8 & 6 & 0 & 0.515947 & -2.504840 & 0.234730 \\
\hline 9 & 6 & 0 & 2.252034 & 1. 326627 & 1. 301124 \\
\hline 10 & 53 & 0 & 2.138001 & 3.555993 & 1. 491999 \\
\hline 11 & 6 & 0 & 1.714534 & -2.471942 & -0.664731 \\
\hline 12 & 6 & 0 & 2. 968344 & -2.691508 & -0.237603 \\
\hline 13 & 6 & 0 & -4.327909 & -0.115717 & -1.223007 \\
\hline 14 & 8 & 0 & -4.976539 & 0.893295 & -0.926138 \\
\hline 15 & 7 & 0 & -4.895694 & -1.178349 & -1.859252 \\
\hline 16 & 6 & 0 & -4.207289 & -2.404856 & -2.242642 \\
\hline 17 & 6 & 0 & -6.312687 & -1.127342 & -2.196967 \\
\hline 18 & 6 & 0 & -2.976811 & -5.012799 & 3.883969 \\
\hline 19 & 6 & 0 & -2.443289 & -3.576721 & 3.834740 \\
\hline 20 & 6 & 0 & -2.186294 & -3.068982 & 2. 408778 \\
\hline 21 & 6 & 0 & -1.663708 & -1.620966 & 2.351460 \\
\hline 22 & 6 & 0 & -1.403462 & 1.921428 & -1.074638 \\
\hline 23 & 8 & 0 & -0.184357 & 1.624315 & -1.181643 \\
\hline 24 & 7 & 0 & -1.914850 & 2.945606 & -1.773705 \\
\hline 25 & 6 & 0 & -3.294729 & 3.429796 & -1.687515 \\
\hline 26 & 6 & 0 & -1.032202 & 3. 757692 & -2.614605 \\
\hline 27 & 1 & 0 & -3.102621 & 1.621336 & 0.284487 \\
\hline 28 & 1 & 0 & -2.263854 & -0.349254 & -1.839882 \\
\hline 29 & 1 & 0 & 0.809472 & -2.770470 & 1. 259568 \\
\hline 30 & 1 & 0 & -0.202205 & -3.260685 & -0.113822 \\
\hline P & 1 & 0 & 2. 399469 & 1. 002020 & 318 \\
\hline
\end{tabular}




\begin{tabular}{|c|c|c|c|c|c|}
\hline 32 & 1 & 0 & 3. 182536 & 1. 174091 & 0.753402 \\
\hline 33 & 1 & 0 & 1.507665 & -2.249023 & -1.710870 \\
\hline 34 & 1 & 0 & -4.136999 & -2.481806 & -3.336593 \\
\hline 35 & 1 & 0 & -4.777065 & -3.268308 & -1.877516 \\
\hline 36 & 1 & 0 & -3.217170 & -2.451670 & -1.796787 \\
\hline 37 & 1 & 0 & -6.713006 & -0.164075 & -1.885387 \\
\hline 38 & 1 & 0 & -6.854370 & -1.933217 & -1.685603 \\
\hline 39 & 1 & 0 & -6.448394 & -1.252308 & -3.279162 \\
\hline 40 & 1 & 0 & -3.154267 & -5.343156 & 4. 914396 \\
\hline 41 & 1 & 0 & -2.268129 & -5.715577 & 3.427226 \\
\hline 42 & 1 & 0 & -3.924745 & -5.101065 & 3.338168 \\
\hline 43 & 1 & 0 & -3.156527 & -2.903250 & 4. 331320 \\
\hline 44 & 1 & 0 & -1.512067 & -3.511160 & 4. 416162 \\
\hline 45 & 1 & 0 & -1.479665 & -3.754432 & 1.916665 \\
\hline 46 & 1 & 0 & -3.117271 & -3.134928 & 1.829046 \\
\hline 47 & 1 & 0 & -2.372411 & -0.982063 & 2. 899956 \\
\hline 48 & 1 & 0 & -0.719086 & -1.552199 & 2.915608 \\
\hline 49 & 1 & 0 & -3.963761 & 2. 678292 & -1.270780 \\
\hline 50 & 1 & 0 & -3.640156 & 3. 667808 & -2.699313 \\
\hline 51 & 1 & 0 & -3.331801 & 4. 347718 & -1.087444 \\
\hline 52 & 1 & 0 & -0.011702 & 3. 391730 & -2.531058 \\
\hline 53 & 1 & 0 & -1.076786 & 4. 801640 & -2.284429 \\
\hline 54 & 1 & 0 & -1.364164 & 3.704866 & -3.657833 \\
\hline 55 & 6 & 0 & 4. 204833 & -2.696276 & -1.033645 \\
\hline 56 & 6 & 0 & 5.437858 & -2.809910 & -0.367480 \\
\hline 57 & 6 & 0 & 4. 219161 & -2.591729 & -2.437451 \\
\hline 58 & 6 & 0 & 6.641914 & -2.808201 & -1.070549 \\
\hline 59 & 1 & 0 & 5.447056 & -2.895225 & 0.716793 \\
\hline 60 & 6 & 0 & 5. 420254 & -2.589752 & -3.140648 \\
\hline 61 & 1 & 0 & 3.283175 & -2.518629 & -2.983636 \\
\hline 62 & 6 & 0 & 6.638238 & -2.696537 & -2.461449 \\
\hline 63 & 1 & 0 & 7. 581614 & -2.894248 & -0.531586 \\
\hline 64 & 1 & 0 & 5.408070 & -2.508572 & -4.224415 \\
\hline 65 & 1 & 0 & 7. 573938 & -2.696160 & -3.013749 \\
\hline 66 & 1 & 0 & 3. 112128 & -2.888663 & 0.825865 \\
\hline
\end{tabular}




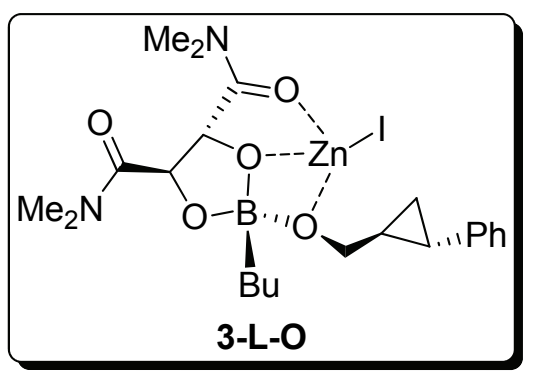

Standard orientation:

\begin{tabular}{|c|c|c|c|c|c|}
\hline \multirow{2}{*}{$\begin{array}{l}\text { Center } \\
\text { Number }\end{array}$} & \multirow{2}{*}{$\begin{array}{l}\text { Atomic } \\
\text { Number }\end{array}$} & \multirow{2}{*}{$\begin{array}{l}\text { Atomic } \\
\text { Type }\end{array}$} & \multicolumn{2}{|c|}{ Coordinates } & $($ Angstroms) \\
\hline & & & X & Y & Z \\
\hline 1 & 6 & 0 & 3. 036238 & -0.062922 & 1. 027121 \\
\hline 2 & 30 & 0 & 0.346118 & 1. 674721 & 0.033061 \\
\hline 3 & 8 & 0 & 0.541938 & 0.882947 & -1.833734 \\
\hline 4 & 6 & 0 & -0.529250 & 0.552560 & -2.409663 \\
\hline 5 & 7 & 0 & -0.539878 & 0.293878 & -3.726073 \\
\hline 6 & 6 & 0 & 0.691872 & 0.418518 & -4.507100 \\
\hline 7 & 8 & 0 & -1.653123 & 1. 061539 & -0.333363 \\
\hline 8 & 5 & 0 & -1.607629 & 0.012603 & 0.787521 \\
\hline 9 & 6 & 0 & -2.700246 & 0.297319 & 1.926330 \\
\hline 10 & 6 & 0 & -2.646990 & -0.649278 & 3.138506 \\
\hline 11 & 6 & 0 & -3.745031 & -0.391294 & 4. 180155 \\
\hline 12 & 6 & 0 & -3.677541 & -1.335156 & 5.385947 \\
\hline 13 & 8 & 0 & -1.721706 & -1.256339 & 0.063125 \\
\hline 14 & 6 & 0 & -2.153550 & -1.067164 & -1.250199 \\
\hline 15 & 6 & 0 & -3.659399 & -1.340476 & -1.540902 \\
\hline 16 & 7 & 0 & -4.317095 & -2.329618 & -0.878434 \\
\hline 17 & 6 & 0 & -5.728908 & -2.544766 & -1.182183 \\
\hline 18 & 6 & 0 & -1.821210 & 0.432952 & -1.586774 \\
\hline 19 & 8 & 0 & -0.175621 & 0.205848 & 1. 295005 \\
\hline 20 & 6 & 0 & 0.654688 & -0.871532 & 1. 743822 \\
\hline 21 & 6 & 0 & 1. 873820 & -1.013138 & 0.865471 \\
\hline 22 & 6 & 0 & 3. 181867 & -1.513128 & 1. 451092 \\
\hline 23 & 1 & 0 & 3. 170905 & -1.677436 & 2.527028 \\
\hline 24 & 53 & 0 & 1. 457809 & 3.893438 & 0.510621 \\
\hline 25 & 6 & 0 & 4. 056594 & -2.471398 & 0.713027 \\
\hline 26 & 8 & 0 & -4.208893 & -0.667149 & -2.422510 \\
\hline 27 & 6 & 0 & -3.726243 & -3.335008 & -0.000166 \\
\hline 28 & 6 & 0 & -1.729422 & -0.061176 & -4.503383 \\
\hline 29 & 1 & 0 & -2.655897 & 0.894341 & -2.116153 \\
\hline 30 & 1 & 0 & -1.597006 & -1.745931 & -1.916092 \\
\hline 31 & 1 & 0 & 0.954120 & -0.664789 & 2. 779664 \\
\hline 32 & 1 & 0 & 0.066230 & -1.793712 & 1. 730420 \\
\hline
\end{tabular}




\begin{tabular}{|c|c|c|c|c|c|}
\hline 33 & 1 & 0 & 2. 987136 & 0.687452 & 1.812368 \\
\hline 34 & 1 & 0 & 3. 573730 & 0.258792 & 0.139452 \\
\hline 35 & 1 & 0 & 1. 654857 & -1.345227 & -0.147704 \\
\hline 36 & 1 & 0 & -3.856557 & -4.331470 & -0.444361 \\
\hline 37 & 1 & 0 & -4.236912 & -3.325748 & 0.970747 \\
\hline 38 & 1 & 0 & -2.672881 & -3.134990 & 0.167191 \\
\hline 39 & 1 & 0 & -6.099559 & -1.705873 & -1.768185 \\
\hline 40 & 1 & 0 & -6.294005 & -2.628161 & -0.246658 \\
\hline 41 & 1 & 0 & -5.865370 & -3.472872 & -1.753786 \\
\hline 42 & 1 & 0 & -4.476576 & -1.126866 & 6.107326 \\
\hline 43 & 1 & 0 & -2.720197 & -1.236534 & 5.913188 \\
\hline 44 & 1 & 0 & -3.774771 & -2.383510 & 5. 075381 \\
\hline 45 & 1 & 0 & -4.728316 & -0.483430 & 3.696297 \\
\hline 46 & 1 & 0 & -3.675291 & 0.650229 & 4.524779 \\
\hline 47 & 1 & 0 & -1.667508 & -0.560710 & 3. 632067 \\
\hline 48 & 1 & 0 & -2.720520 & -1.694166 & 2. 798609 \\
\hline 49 & 1 & 0 & -3.704118 & 0.241805 & 1. 474992 \\
\hline 50 & 1 & 0 & -2.593636 & 1. 334211 & 2. 280631 \\
\hline 51 & 1 & 0 & -2.606052 & -0.219087 & -3.874884 \\
\hline 52 & 1 & 0 & -1.527960 & -0.982438 & -5.062442 \\
\hline 53 & 1 & 0 & -1.946044 & 0.736377 & -5.224421 \\
\hline 54 & 1 & 0 & 1. 503402 & 0.735272 & -3.855932 \\
\hline 55 & 1 & 0 & 0.547309 & 1. 158660 & -5.302287 \\
\hline 56 & 1 & 0 & 0.938069 & -0.545612 & -4.966657 \\
\hline 57 & 6 & 0 & 4. 736015 & -3.472099 & 1. 423102 \\
\hline 58 & 6 & 0 & 5.563362 & -4.386400 & 0.769435 \\
\hline 59 & 6 & 0 & 5. 728641 & -4.318436 & -0.614371 \\
\hline 60 & 6 & 0 & 5. 058452 & -3.326805 & -1.334716 \\
\hline 61 & 6 & 0 & 4. 232581 & -2.414507 & -0.679040 \\
\hline 62 & 1 & 0 & 4. 615548 & -3.532308 & 2.502465 \\
\hline 63 & 1 & 0 & 6. 079155 & -5.151154 & 1. 344391 \\
\hline 64 & 1 & 0 & 6. 373411 & -5.027343 & -1.126527 \\
\hline 65 & 1 & 0 & 5. 181284 & -3.260876 & -2.412896 \\
\hline 66 & 1 & 0 & 3. 724782 & -1.648315 & -1.260057 \\
\hline
\end{tabular}




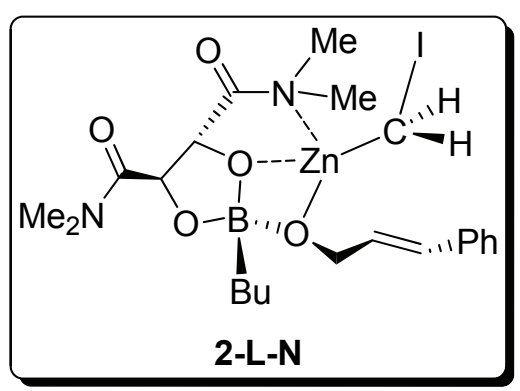

Standard orientation:

\begin{tabular}{|c|c|c|c|c|c|}
\hline \multirow{2}{*}{$\begin{array}{l}\text { Center } \\
\text { Number }\end{array}$} & \multirow{2}{*}{$\begin{array}{l}\text { Atomic } \\
\text { Number }\end{array}$} & \multirow{2}{*}{$\begin{array}{l}\text { Atomic } \\
\text { Type }\end{array}$} & \multicolumn{2}{|c|}{ Coordinates } & \multirow{2}{*}{$\begin{array}{r}(\text { Angstroms) } \\
Z\end{array}$} \\
\hline & & & X & Y & \\
\hline 1 & 6 & 0 & 1.803234 & -0.047282 & -1.753954 \\
\hline 2 & 6 & 0 & 2.838346 & 0.950205 & -1.191822 \\
\hline 3 & 8 & 0 & 2. 898971 & 0.703432 & 0.199904 \\
\hline 4 & 5 & 0 & 2.083608 & -0.430340 & 0.560954 \\
\hline 5 & 8 & 0 & 1.680312 & -1.077014 & -0.763231 \\
\hline 6 & 8 & 0 & 0.651556 & 0.030835 & 1. 001476 \\
\hline 7 & 30 & 0 & -0.351929 & -1.311832 & -0.080470 \\
\hline 8 & 6 & 0 & 0.374882 & 1. 098422 & 1.914312 \\
\hline 9 & 6 & 0 & -1.399718 & -2.923042 & 0.399092 \\
\hline 10 & 53 & 0 & -3.527023 & -2.858659 & -0.282663 \\
\hline 11 & 6 & 0 & -0.625318 & 2.038913 & 1.312403 \\
\hline 12 & 6 & 0 & -1.847666 & 2.260337 & 1. 822319 \\
\hline 13 & 6 & 0 & 4. 207628 & 0.747112 & -1.893326 \\
\hline 14 & 8 & 0 & 4. 286887 & 0.030602 & -2.893701 \\
\hline 15 & 7 & 0 & 5.286026 & 1. 414043 & -1.388013 \\
\hline 16 & 6 & 0 & 5.261682 & 2.368519 & -0.288142 \\
\hline 17 & 6 & 0 & 6.578450 & 1. 254603 & -2.041238 \\
\hline 18 & 6 & 0 & 4. 397835 & -1.249575 & 5. 163961 \\
\hline 19 & 6 & 0 & 3.934129 & -1.863343 & 3.838439 \\
\hline 20 & 6 & 0 & 3.212333 & -0.864850 & 2.922118 \\
\hline 21 & 6 & 0 & 2.743156 & -1.470871 & 1. 587161 \\
\hline 22 & 6 & 0 & 0.448784 & 0.620335 & -2.023428 \\
\hline 23 & 8 & 0 & 0.291650 & 1.815378 & -2.160496 \\
\hline 24 & 7 & 0 & -0.674949 & -0.279523 & -2.038008 \\
\hline 25 & 6 & 0 & -0.537177 & -1.420799 & -2.989129 \\
\hline 26 & 6 & 0 & -1.968471 & 0.420681 & -2.213555 \\
\hline 27 & 1 & 0 & 2. 195248 & -0.478958 & -2.681170 \\
\hline 28 & 1 & 0 & 2.503485 & 1.976917 & -1.387209 \\
\hline 29 & 1 & 0 & 0.007175 & 0.689192 & 2.864634 \\
\hline 30 & 1 & 0 & 1. 322556 & 1. 616131 & 2. 103642 \\
\hline . & 1 & 0 & -1.028740 & -3.850219 & -0.039868 \\
\hline
\end{tabular}




\begin{tabular}{|c|c|c|c|c|c|}
\hline 32 & 1 & 0 & -1.493265 & -3.070859 & 1. 475411 \\
\hline 33 & 1 & 0 & -0.298696 & 2. 534568 & 0.400135 \\
\hline 34 & 1 & 0 & 5. 489492 & 3.379500 & -0.654973 \\
\hline 35 & 1 & 0 & 6.026325 & 2. 096714 & 0.450890 \\
\hline 36 & 1 & 0 & 4. 298614 & 2. 363513 & 0.214349 \\
\hline 37 & 1 & 0 & 6. 479534 & 0.517224 & -2.836184 \\
\hline 38 & 1 & 0 & 7. 328950 & 0.919911 & -1.313876 \\
\hline 39 & 1 & 0 & 6.914143 & 2. 209307 & -2.468669 \\
\hline 40 & 1 & 0 & 4. 913888 & -1.985966 & 5. 791364 \\
\hline 41 & 1 & 0 & 3.548655 & -0.859604 & 5.739557 \\
\hline 42 & 1 & 0 & 5. 089518 & -0.414924 & 4. 992924 \\
\hline 43 & 1 & 0 & 4. 800217 & -2.278739 & 3. 303453 \\
\hline 44 & 1 & 0 & 3. 267899 & -2.714539 & 4. 040878 \\
\hline 45 & 1 & 0 & 2. 352358 & -0.447801 & 3.468343 \\
\hline 46 & 1 & 0 & 3. 882332 & -0.016409 & 2. 720397 \\
\hline 47 & 1 & 0 & 3. 607929 & -1.932278 & 1. 086193 \\
\hline 48 & 1 & 0 & 2. 047799 & -2.300008 & 1. 798545 \\
\hline 49 & 1 & 0 & 0.392516 & -1.960264 & -2.805325 \\
\hline 50 & 1 & 0 & -0.552383 & -1.060909 & -4.025458 \\
\hline 51 & 1 & 0 & -1.378007 & -2.100252 & -2.834136 \\
\hline 52 & 1 & 0 & -2.081583 & 1. 181106 & -1.440808 \\
\hline 53 & 1 & 0 & -2.768357 & -0.314900 & -2.118511 \\
\hline 54 & 1 & 0 & -2.017689 & 0.907225 & -3.194286 \\
\hline 55 & 6 & 0 & -2.890678 & 3. 151581 & 1. 290618 \\
\hline 56 & 6 & 0 & -4.096147 & 3. 284114 & 2.001721 \\
\hline 57 & 6 & 0 & -2.744563 & 3. 878991 & 0.092949 \\
\hline 58 & 6 & 0 & -5.119345 & 4. 112142 & 1. 541361 \\
\hline 59 & 1 & 0 & -4.228065 & 2. 727879 & 2.927028 \\
\hline 60 & 6 & 0 & -3.766844 & 4. 703834 & -0.368140 \\
\hline 61 & 1 & 0 & -1.827718 & 3. 797172 & -0.483919 \\
\hline 62 & 6 & 0 & -4.958819 & 4. 826261 & 0.353378 \\
\hline 63 & 1 & 0 & -6.041283 & 4. 197855 & 2. 110253 \\
\hline 64 & 1 & 0 & -3.633567 & 5.255893 & -1.294806 \\
\hline 65 & 1 & 0 & -5.753698 & 5.472060 & -0.009520 \\
\hline 66 & 1 & 0 & -2.120337 & 1. 734797 & 2. 739145 \\
\hline
\end{tabular}




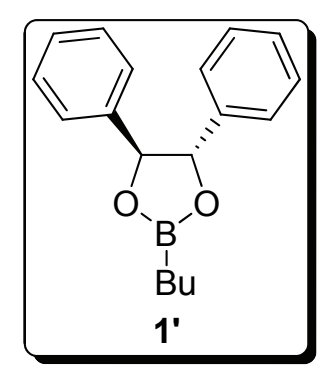

Standard orientation:

\begin{tabular}{|c|c|c|c|c|c|}
\hline \multirow{2}{*}{$\begin{array}{l}\text { Center } \\
\text { Number }\end{array}$} & \multirow{2}{*}{$\begin{array}{l}\text { Atomic } \\
\text { Number }\end{array}$} & \multirow{2}{*}{$\begin{array}{l}\text { Atomic } \\
\text { Type }\end{array}$} & \multicolumn{2}{|c|}{ Coordinates } & (Angstroms) \\
\hline & & & $X$ & Y & Z \\
\hline 1 & 6 & 0 & -0.220946 & 0.412505 & 0.396145 \\
\hline 2 & 6 & 0 & -0.655355 & -0.581393 & -0.741822 \\
\hline 3 & 8 & 0 & 0.555879 & -1.291954 & -1.051977 \\
\hline 4 & 5 & 0 & 1. 616094 & -0.605299 & -0.503479 \\
\hline 5 & 8 & 0 & 1. 212775 & 0.445979 & 0.288185 \\
\hline 6 & 6 & 0 & 6.593501 & -0.174082 & 0.900073 \\
\hline 7 & 6 & 0 & 5. 589124 & -0.739688 & -0.109605 \\
\hline 8 & 6 & 0 & 4. 131641 & -0.404836 & 0.233270 \\
\hline 9 & 6 & 0 & 3.118842 & -0.973414 & -0.776278 \\
\hline 10 & 1 & 0 & -0.473355 & -0.022395 & 1. 371481 \\
\hline 11 & 1 & 0 & -0.944952 & -0.006480 & -1.630595 \\
\hline 12 & 1 & 0 & 7. 623997 & -0.431422 & 0.628862 \\
\hline 13 & 1 & 0 & 6.406150 & -0.566560 & 1. 907384 \\
\hline 14 & 1 & 0 & 6.526418 & 0.919601 & 0.955272 \\
\hline 15 & 1 & 0 & 5.820869 & -0.354488 & -1.113280 \\
\hline 16 & 1 & 0 & 5.705192 & -1.831532 & -0.170742 \\
\hline 17 & 1 & 0 & 3.901559 & -0.784945 & 1. 238897 \\
\hline 18 & 1 & 0 & 4. 012790 & 0.685446 & 0.295510 \\
\hline 19 & 1 & 0 & 3. 371459 & -0.617292 & -1.788878 \\
\hline 20 & 1 & 0 & 3. 210684 & -2.067641 & -0.837867 \\
\hline 21 & 6 & 0 & -0.834678 & 1. 788259 & 0.284097 \\
\hline 22 & 6 & 0 & -0.234443 & 2. 787329 & -0.491985 \\
\hline 23 & 6 & 0 & -2.048238 & 2. 062743 & 0.927397 \\
\hline 24 & 6 & 0 & -0.840254 & 4. 037158 & -0.624693 \\
\hline 25 & 1 & 0 & 0.718277 & 2.586852 & -0.971970 \\
\hline 26 & 6 & 0 & -2.658589 & 3.310514 & 0.787968 \\
\hline 27 & 1 & 0 & -2.515993 & 1. 297006 & 1.541618 \\
\hline 28 & 6 & 0 & -2.055711 & 4. 301466 & 0.011117 \\
\hline 29 & 1 & 0 & -0.359949 & 4. 807457 & -1.222433 \\
\hline 30 & 1 & 0 & -3.599285 & 3.510422 & 1. 294220 \\
\hline 31 & 1 & 0 & -2.525598 & 5.275915 & -0.091921 \\
\hline 32 & 6 & 0 & -1.780458 & -1.512087 & -0.3557 \\
\hline
\end{tabular}




$\begin{array}{rrrrrr}33 & 6 & 0 & -3.105403 & -1.159782 & -0.641523 \\ 34 & 6 & 0 & -1.526728 & -2.707606 & 0.328275 \\ 35 & 6 & 0 & -4.160910 & -1.980174 & -0.238672 \\ 36 & 1 & 0 & -3.311393 & -0.239807 & -1.183487 \\ 37 & 6 & 0 & -2.580698 & -3.531495 & 0.723848 \\ 38 & 1 & 0 & -0.500216 & -2.998689 & 0.528592 \\ 39 & 6 & 0 & -3.900885 & -3.168616 & 0.445745 \\ 40 & 1 & 0 & -2.370565 & -4.461046 & 1.246464 \\ 41 & 1 & 0 & -4.720590 & -3.812192 & 0.753707 \\ 42 & 1 & 0 & & \end{array}$

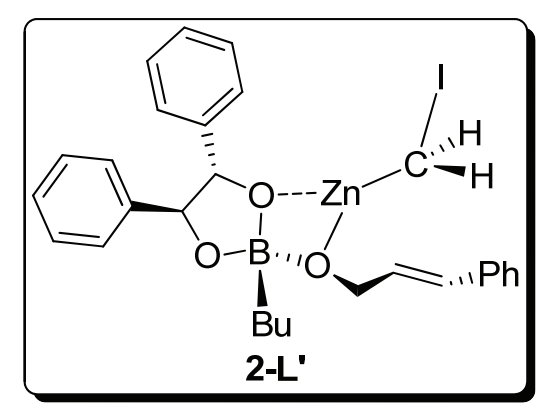

Standard orientation:

\begin{tabular}{|c|c|c|c|c|c|}
\hline \multirow{2}{*}{$\begin{array}{l}\text { Center } \\
\text { Number }\end{array}$} & \multirow{2}{*}{$\begin{array}{l}\text { Atomic } \\
\text { Number }\end{array}$} & \multirow{2}{*}{$\begin{array}{l}\text { Atomic } \\
\text { Type }\end{array}$} & \multicolumn{2}{|c|}{ Coordinates } & \multirow{2}{*}{$($ Angstroms) } \\
\hline & & & $X$ & $\mathrm{Y}$ & \\
\hline 1 & 6 & 0 & -2.490386 & -0.822056 & -0.180076 \\
\hline 2 & 6 & 0 & -2.806237 & 0.354228 & 0.792053 \\
\hline 3 & 8 & 0 & -2.460235 & 1. 501873 & 0.036657 \\
\hline 4 & 5 & 0 & -1.353583 & 1. 214159 & -0.827522 \\
\hline 5 & 8 & 0 & -1.357429 & -0.332528 & -0.937102 \\
\hline 6 & 8 & 0 & 0.017840 & 1. 294520 & -0.080563 \\
\hline 7 & 30 & 0 & 0.523131 & -0.600423 & -0.242878 \\
\hline 8 & 6 & 0 & 0.742060 & 2.511385 & 0.133662 \\
\hline 9 & 6 & 0 & 2.006913 & -1.861894 & -0.054083 \\
\hline 10 & 53 & 0 & 2. 710694 & -2.433119 & -2.094697 \\
\hline 11 & 6 & 0 & 1.855240 & 2. 270839 & 1. 106634 \\
\hline 12 & 6 & 0 & 3. 154266 & 2. 397953 & 0.790179 \\
\hline 13 & 6 & 0 & -2.296906 & 5.595319 & -3.518146 \\
\hline 14 & 6 & 0 & -1.902854 & 4. 115219 & -3.574662 \\
\hline 15 & 6 & 0 & -1.763886 & 3. 466680 & -2.190096 \\
\hline 16 & 6 & 0 & -1.368727 & 1. 978585 & -2.239727 \\
\hline 17 & 1 & 0 & -3.324622 & -0.914832 & -0.888761 \\
\hline 18 & 1 & 0 & -2.154005 & 0.264420 & 1. 677779 \\
\hline 19 & 1 & 0 & 1. 133850 & 2.887635 & -0.820339 \\
\hline
\end{tabular}




\begin{tabular}{|c|c|c|c|c|c|}
\hline 20 & 1 & 0 & 0.033244 & 3. 251342 & 0.527418 \\
\hline 21 & 1 & 0 & 2. 897351 & -1.457703 & 0.426437 \\
\hline 22 & 1 & 0 & 1. 739423 & -2.812292 & 0.405815 \\
\hline 23 & 1 & 0 & 1. 547491 & 1. 971665 & 2. 107971 \\
\hline 24 & 1 & 0 & -2.393136 & 6.026210 & -4.521894 \\
\hline 25 & 1 & 0 & -1.548081 & 6.184873 & -2.973909 \\
\hline 26 & 1 & 0 & -3.256940 & 5.729664 & -3.004199 \\
\hline 27 & 1 & 0 & -2.651113 & 3.558809 & -4.157611 \\
\hline 28 & 1 & 0 & -0.954744 & 4. 008403 & -4.121897 \\
\hline 29 & 1 & 0 & -1.026119 & 4. 039936 & -1.608392 \\
\hline 30 & 1 & 0 & -2.712184 & 3.565094 & -1.644710 \\
\hline 31 & 1 & 0 & -2.094426 & 1. 454810 & -2.881855 \\
\hline 32 & 1 & 0 & -0.400909 & 1. 872881 & -2.756861 \\
\hline 33 & 6 & 0 & 4. 319436 & 2. 190826 & 1. 662886 \\
\hline 34 & 6 & 0 & 5.605071 & 2. 233025 & 1.094995 \\
\hline 35 & 6 & 0 & 4. 214278 & 1.949296 & 3.045862 \\
\hline 36 & 6 & 0 & 6.744746 & 2. 030454 & 1. 872227 \\
\hline 37 & 1 & 0 & 5.706468 & 2. 421841 & 0.028741 \\
\hline 38 & 6 & 0 & 5.351160 & 1. 746908 & 3.822818 \\
\hline 39 & 1 & 0 & 3.236852 & 1. 928600 & 3.519184 \\
\hline 40 & 6 & 0 & 6.622242 & 1. 784915 & 3.240367 \\
\hline 41 & 1 & 0 & 7. 726974 & 2. 065037 & 1. 408784 \\
\hline 42 & 1 & 0 & 5.247435 & 1.563464 & 4.889003 \\
\hline 43 & 1 & 0 & 7. 507601 & 1. 628387 & 3.850383 \\
\hline 44 & 1 & 0 & 3. 398733 & 2. 684397 & -0.233934 \\
\hline 45 & 6 & 0 & -2.171925 & -2.186676 & 0.399798 \\
\hline 46 & 6 & 0 & -1.481799 & -3.115699 & -0.398668 \\
\hline 47 & 6 & 0 & -2.565400 & -2.574334 & 1. 687907 \\
\hline 48 & 6 & 0 & -1.174072 & -4.387184 & 0.086328 \\
\hline 49 & 1 & 0 & -1.191517 & -2.841054 & -1.408851 \\
\hline 50 & 6 & 0 & -2.261067 & -3.849024 & 2.171356 \\
\hline 51 & 1 & 0 & -3.125262 & -1.889225 & 2. 314262 \\
\hline 52 & 6 & 0 & -1.560729 & -4.757040 & 1. 376496 \\
\hline 53 & 1 & 0 & -0.637713 & -5.088328 & -0.547178 \\
\hline 54 & 1 & 0 & -2.573676 & -4.129972 & 3. 173562 \\
\hline 55 & 1 & 0 & -1.323414 & -5.747129 & 1.756107 \\
\hline 56 & 6 & 0 & -4.248710 & 0.445947 & 1. 248623 \\
\hline 57 & 6 & 0 & -4.586209 & 0.337738 & 2.602222 \\
\hline 58 & 6 & 0 & -5.270706 & 0.670053 & 0.315628 \\
\hline 59 & 6 & 0 & -5.917347 & 0.422651 & 3. 017713 \\
\hline 60 & 1 & 0 & -3.799240 & 0.199306 & 3. 341091 \\
\hline 61 & 6 & 0 & -6.600023 & 0.756567 & 0.726168 \\
\hline 62 & 1 & 0 & -5.015029 & 0.802338 & -0.731719 \\
\hline 63 & 6 & 0 & -6.928489 & 0.627412 & 2. 078815 \\
\hline
\end{tabular}




\begin{tabular}{|c|c|c|c|c|c|}
\hline 64 & 1 & 0 & -6.160695 & 0.337820 & 4. 073567 \\
\hline 65 & 1 & 0 & -7.381433 & 0.931296 & -0.008813 \\
\hline 66 & 1 & 0 & -7.965001 & 0.696040 & 2. 398109 \\
\hline
\end{tabular}

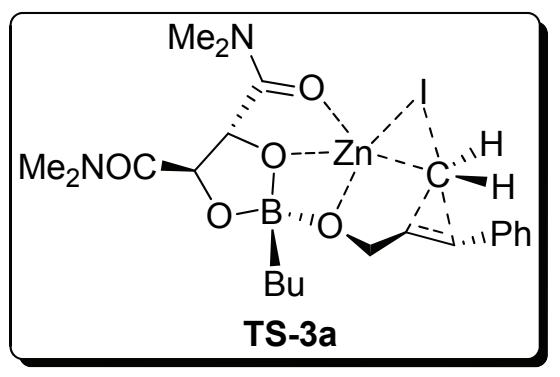

Imaginary frequency: $-361.7 \mathrm{~cm}^{-1}$

Standard orientation:

\begin{tabular}{|c|c|c|c|c|c|}
\hline \multirow{2}{*}{$\begin{array}{l}\text { Center } \\
\text { Number }\end{array}$} & \multirow{2}{*}{$\begin{array}{l}\text { Atomic } \\
\text { Number }\end{array}$} & \multirow{2}{*}{$\begin{array}{l}\text { Atomic } \\
\text { Type }\end{array}$} & \multicolumn{2}{|c|}{ Coordinates } & \multirow{2}{*}{$\begin{array}{r}(\text { Angstroms) } \\
Z\end{array}$} \\
\hline & & & $X$ & Y & \\
\hline 1 & 6 & 0 & 2. 426072 & -0.256063 & -0.451510 \\
\hline 2 & 30 & 0 & 0.544842 & -0.776244 & -0.036675 \\
\hline 3 & 8 & 0 & -0.449938 & -2.060036 & 1. 159969 \\
\hline 4 & 6 & 0 & -1.673240 & -2.197932 & 0.892214 \\
\hline 5 & 7 & 0 & -2.357704 & -3.245058 & 1.376425 \\
\hline 6 & 6 & 0 & -1.677468 & -4.247758 & 2. 197280 \\
\hline 7 & 8 & 0 & -1.407812 & -0.349207 & -0.628232 \\
\hline 8 & 5 & 0 & -1.422008 & 1. 101922 & -0.072877 \\
\hline 9 & 6 & 0 & -1.667709 & 2. 169830 & -1.252706 \\
\hline 10 & 6 & 0 & -1.934549 & 3.620474 & -0.811196 \\
\hline 11 & 6 & 0 & -2.266381 & 4. 571732 & -1.970365 \\
\hline 12 & 6 & 0 & -2.541532 & 6.010898 & -1.520726 \\
\hline 13 & 8 & 0 & -2.481863 & 1. 031261 & 0.939563 \\
\hline 14 & 6 & 0 & -3.223683 & -0.145305 & 0.880968 \\
\hline 15 & 6 & 0 & -4.655589 & -0.008916 & 0.284713 \\
\hline 16 & 7 & 0 & -5.415589 & 1. 069380 & 0.622529 \\
\hline 17 & 6 & 0 & -6.759076 & 1. 176840 & 0.064344 \\
\hline 18 & 6 & 0 & -2.374898 & -1.147801 & 0.014946 \\
\hline 19 & 8 & 0 & -0.087451 & 1. 161175 & 0.624338 \\
\hline 20 & 6 & 0 & 0.918256 & 2. 131881 & 0.415532 \\
\hline 21 & 6 & 0 & 2. 269240 & 1.579057 & 0.813759 \\
\hline 22 & 6 & 0 & 3. 441862 & 2. 028316 & 0.273905 \\
\hline 23 & 1 & 0 & 3.375140 & 2.605237 & -0.649217 \\
\hline 24 & 53 & 0 & 1. 978275 & -2.651940 & -1.793704 \\
\hline 25 & 6 & 0 & 4. 798576 & 1.818308 & 0.771999 \\
\hline 26 & 8 & 0 & -5.090481 & -0.912775 & -0.439994 \\
\hline
\end{tabular}




\begin{tabular}{|c|c|c|c|c|c|}
\hline 27 & 6 & 0 & -5.086732 & 2. 081644 & 1. 620820 \\
\hline 28 & 6 & 0 & -3.773952 & -3.512552 & 1. 122012 \\
\hline 29 & 1 & 0 & -3.009074 & -1.631781 & -0.729652 \\
\hline 30 & 1 & 0 & -3.361298 & -0.542632 & 1. 901050 \\
\hline 31 & 1 & 0 & 0.945143 & 2. 478813 & -0.626067 \\
\hline 32 & 1 & 0 & 0.708948 & 3.008352 & 1. 048076 \\
\hline 33 & 1 & 0 & 2. 660290 & 0.217500 & -1.403468 \\
\hline 34 & 1 & 0 & 3. 319281 & -0.664214 & 0.016329 \\
\hline 35 & 1 & 0 & 2. 278794 & 1. 065267 & 1. 773408 \\
\hline 36 & 1 & 0 & -5.799314 & 2. 023326 & 2. 455577 \\
\hline 37 & 1 & 0 & -5.168294 & 3.080817 & 1. 174616 \\
\hline 38 & 1 & 0 & -4.071178 & 1. 956808 & 1.984257 \\
\hline 39 & 1 & 0 & -6.887128 & 0.413087 & -0.700604 \\
\hline 40 & 1 & 0 & -6.897988 & 2. 172677 & -0.373337 \\
\hline 41 & 1 & 0 & -7.515723 & 1. 035252 & 0.848217 \\
\hline 42 & 1 & 0 & -2.774976 & 6.662597 & -2.371067 \\
\hline 43 & 1 & 0 & -1.672929 & 6.438033 & -1.003334 \\
\hline 44 & 1 & 0 & -3.390096 & 6.053805 & -0.826014 \\
\hline 45 & 1 & 0 & -3.139337 & 4. 183168 & -2.514311 \\
\hline 46 & 1 & 0 & -1.436190 & 4. 565512 & -2.691573 \\
\hline 47 & 1 & 0 & -1.067839 & 4. 024533 & -0.266218 \\
\hline 48 & 1 & 0 & -2.764653 & 3. 629632 & -0.090471 \\
\hline 49 & 1 & 0 & -2.542352 & 1.818449 & -1.822009 \\
\hline 50 & 1 & 0 & -0.839971 & 2. 157142 & -1.980948 \\
\hline 51 & 1 & 0 & -4.249424 & -2.721117 & 0.542949 \\
\hline 52 & 1 & 0 & -4.299282 & -3.612217 & 2. 079547 \\
\hline 53 & 1 & 0 & -3.872940 & -4.458966 & 0.576947 \\
\hline 54 & 1 & 0 & -0.613547 & -4.024613 & 2. 232894 \\
\hline 55 & 1 & 0 & -1.831621 & -5.239584 & 1.758270 \\
\hline 56 & 1 & 0 & -2.091835 & -4.244767 & 3.212479 \\
\hline 57 & 6 & 0 & 5.882457 & 2. 266309 & -0.008740 \\
\hline 58 & 6 & 0 & 7. 197213 & 2. 092686 & 0.416574 \\
\hline 59 & 6 & 0 & 7. 460389 & 1.464716 & 1.635518 \\
\hline 60 & 6 & 0 & 6.396449 & 1. 017107 & 2.425976 \\
\hline 61 & 6 & 0 & 5. 082767 & 1. 193536 & 2. 004055 \\
\hline 62 & 1 & 0 & 5.682808 & 2. 751197 & -0.961424 \\
\hline 63 & 1 & 0 & 8. 016139 & 2. 445004 & -0.204459 \\
\hline 64 & 1 & 0 & 8. 484667 & 1. 326547 & 1.970073 \\
\hline 65 & 1 & 0 & 6.593737 & 0.532146 & 3.378135 \\
\hline 66 & 1 & 0 & 4. 270816 & 0.849898 & 2. 638221 \\
\hline
\end{tabular}




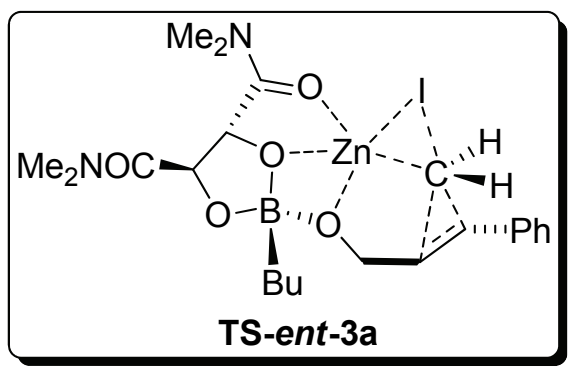

Imaginary frequency: $-302.9 \mathrm{~cm}^{-1}$

Standard orientation:

\begin{tabular}{|c|c|c|c|c|c|}
\hline \multirow{2}{*}{$\begin{array}{l}\text { Center } \\
\text { Number }\end{array}$} & \multirow{2}{*}{$\begin{array}{l}\text { Atomic } \\
\text { Number }\end{array}$} & \multirow{2}{*}{$\begin{array}{l}\text { Atomic } \\
\text { Type }\end{array}$} & \multicolumn{2}{|c|}{ Coordinates } & \multirow{2}{*}{$\begin{array}{r}(\text { Angstroms) } \\
Z\end{array}$} \\
\hline & & & $X$ & Y & \\
\hline 1 & 6 & 0 & 2.528119 & -0.052491 & -0.866165 \\
\hline 2 & 30 & 0 & 0.751103 & -0.782799 & -0.383999 \\
\hline 3 & 8 & 0 & 0.110940 & -1.879061 & 1. 205475 \\
\hline 4 & 6 & 0 & -1.116736 & -2.151713 & 1. 169588 \\
\hline 5 & 7 & 0 & -1.617046 & -3.153411 & 1. 910031 \\
\hline 6 & 6 & 0 & -0.727346 & -3.961989 & 2. 743873 \\
\hline 7 & 8 & 0 & -1.265402 & -0.553252 & -0.623544 \\
\hline 8 & 5 & 0 & -1.400678 & 0.985926 & -0.323392 \\
\hline 9 & 6 & 0 & -2.148795 & 1. 716321 & -1.551976 \\
\hline 10 & 6 & 0 & -2.544484 & 3. 186092 & -1.319801 \\
\hline 11 & 6 & 0 & -3.324310 & 3.813772 & -2.483982 \\
\hline 12 & 6 & 0 & -3.723275 & 5.272890 & -2.236752 \\
\hline 13 & 8 & 0 & -2.139459 & 0.960479 & 0.946377 \\
\hline 14 & 6 & 0 & -2.814878 & -0.239812 & 1. 142889 \\
\hline 15 & 6 & 0 & -4.328169 & -0.258500 & 0.774184 \\
\hline 16 & 7 & 0 & -5.103103 & 0.814252 & 1. 097460 \\
\hline 17 & 6 & 0 & -6.524161 & 0.767998 & 0.771056 \\
\hline 18 & 6 & 0 & -2.041308 & -1.306657 & 0.282733 \\
\hline 19 & 8 & 0 & -0.006614 & 1. 404951 & -0.022336 \\
\hline 20 & 6 & 0 & 0.771339 & 2. 171504 & -0.906195 \\
\hline 21 & 6 & 0 & 2. 244983 & 2. 091531 & -0.560944 \\
\hline 22 & 6 & 0 & 2. 728524 & 1.960201 & 0.711806 \\
\hline 23 & 1 & 0 & 2. 011652 & 1. 635746 & 1. 464439 \\
\hline 24 & 53 & 0 & 2. 122274 & -2.755871 & -1.952588 \\
\hline 25 & 6 & 0 & 4. 092076 & 2. 171819 & 1. 180963 \\
\hline 26 & 8 & 0 & -4.802512 & -1.272440 & 0.247723 \\
\hline 27 & 6 & 0 & -4.700474 & 1. 944600 & 1. 927971 \\
\hline 28 & 6 & 0 & -3.020367 & -3.568006 & 1. 918785 \\
\hline 29 & 1 & 0 & -2.744466 & -1.936102 & -0.264516 \\
\hline 30 & 1 & 0 & -2.769829 & -0.503842 & 2.212556 \\
\hline 31 & 1 & 0 & 0.641732 & 1. 857573 & -1.951666 \\
\hline
\end{tabular}




\begin{tabular}{|c|c|c|c|c|c|}
\hline 32 & 1 & 0 & 0.479437 & 3. 233012 & -0.860512 \\
\hline 33 & 1 & 0 & 2. 849360 & 0.202015 & -1.878057 \\
\hline 34 & 1 & 0 & 3. 402960 & -0.329673 & -0.273654 \\
\hline 35 & 1 & 0 & 2. 899040 & 2. 485465 & -1.337779 \\
\hline 36 & 1 & 0 & -5.213794 & 1. 899261 & 2. 899307 \\
\hline 37 & 1 & 0 & -4.990264 & 2. 880988 & 1. 435846 \\
\hline 38 & 1 & 0 & -3.623604 & 1. 955412 & 2. 070012 \\
\hline 39 & 1 & 0 & -6.707438 & -0.071836 & 0.103127 \\
\hline 40 & 1 & 0 & -6.819502 & 1. 705328 & 0.285160 \\
\hline 41 & 1 & 0 & -7.128862 & 0.644266 & 1. 680262 \\
\hline 42 & 1 & 0 & -4.281362 & 5.688809 & -3.084034 \\
\hline 43 & 1 & 0 & -2.839922 & 5.904725 & -2.078471 \\
\hline 44 & 1 & 0 & -4.355775 & 5. 365347 & -1.344460 \\
\hline 45 & 1 & 0 & -4.225821 & 3.214253 & -2.676614 \\
\hline 46 & 1 & 0 & -2.719985 & 3.749401 & -3.400482 \\
\hline 47 & 1 & 0 & -1.650971 & 3. 799443 & -1.126853 \\
\hline 48 & 1 & 0 & -3.149293 & 3. 256663 & -0.404578 \\
\hline 49 & 1 & 0 & -3.065483 & 1. 144295 & -1.766025 \\
\hline 50 & 1 & 0 & -1.557764 & 1. 642820 & -2.479221 \\
\hline 51 & 1 & 0 & -3.653742 & -2.902053 & 1. 333193 \\
\hline 52 & 1 & 0 & -3.385146 & -3.579778 & 2.952860 \\
\hline 53 & 1 & 0 & -3.104716 & -4.584032 & 1. 514338 \\
\hline 54 & 1 & 0 & 0.296586 & -3.617022 & 2. 618883 \\
\hline 55 & 1 & 0 & -0.799882 & -5.013346 & 2. 443237 \\
\hline 56 & 1 & 0 & -1.023487 & -3.876102 & 3. 795977 \\
\hline 57 & 6 & 0 & 4. 391724 & 1. 876450 & 2. 526087 \\
\hline 58 & 6 & 0 & 5.673102 & 2. 070043 & 3.036553 \\
\hline 59 & 6 & 0 & 6.685433 & 2.563436 & 2.211470 \\
\hline 60 & 6 & 0 & 6. 405401 & 2. 864511 & 0.873447 \\
\hline 61 & 6 & 0 & 5. 126333 & 2.674634 & 0.363542 \\
\hline 62 & 1 & 0 & 3.604846 & 1. 490701 & 3.169596 \\
\hline 63 & 1 & 0 & 5.881740 & 1. 834765 & 4. 076405 \\
\hline 64 & 1 & 0 & 7. 686305 & 2. 715351 & 2.605615 \\
\hline 65 & 1 & 0 & 7. 189301 & 3. 251784 & 0.228567 \\
\hline 66 & 1 & 0 & 4. 926964 & 2. 920760 & -0.675165 \\
\hline
\end{tabular}




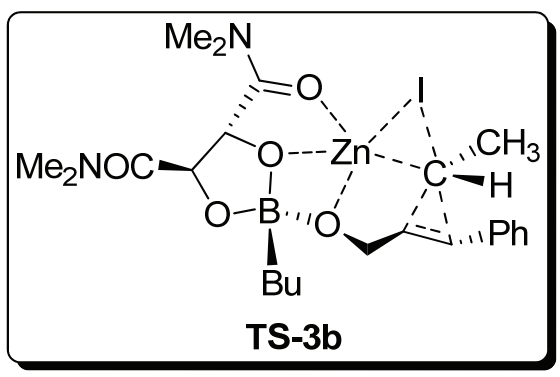

Imaginary frequency: $-251.1 \mathrm{~cm}^{-1}$

Standard orientation:

\begin{tabular}{|c|c|c|c|c|c|}
\hline \multirow{2}{*}{$\begin{array}{l}\text { Center } \\
\text { Number }\end{array}$} & \multirow{2}{*}{$\begin{array}{l}\text { Atomic } \\
\text { Number }\end{array}$} & \multirow{2}{*}{$\begin{array}{l}\text { Atomic } \\
\text { Type }\end{array}$} & \multicolumn{2}{|c|}{ Coordinates } & (Angstroms) \\
\hline & & & X & Y & Z \\
\hline 1 & 6 & 0 & -2.399325 & -0.721531 & 0.095180 \\
\hline 2 & 30 & 0 & -0.370049 & -0.906555 & 0.237902 \\
\hline 3 & 8 & 0 & 0.788573 & -1.878246 & -1.139920 \\
\hline 4 & 6 & 0 & 2. 016182 & -1.932935 & -0.856457 \\
\hline 5 & 7 & 0 & 2. 769638 & -2.944926 & -1.308941 \\
\hline 6 & 6 & 0 & 2. 142188 & -4.029590 & -2.066735 \\
\hline 7 & 8 & 0 & 1. 605140 & -0.356421 & 0.892114 \\
\hline 8 & 5 & 0 & 1. 392728 & 1. 157561 & 0.742424 \\
\hline 9 & 6 & 0 & 1. 353017 & 1.944595 & 2. 142546 \\
\hline 10 & 6 & 0 & 1.503712 & 3.474531 & 2.050675 \\
\hline 11 & 6 & 0 & 1. 570553 & 4. 175369 & 3.414898 \\
\hline 12 & 6 & 0 & 1.731501 & 5.696252 & 3. 313292 \\
\hline 13 & 8 & 0 & 2. 498224 & 1. 558155 & -0.119292 \\
\hline 14 & 6 & 0 & 2.940165 & 0.465226 & -0.880281 \\
\hline 15 & 6 & 0 & 4. 443143 & 0.613072 & -1.189688 \\
\hline 16 & 7 & 0 & 4. 819845 & 1.690816 & -1.934900 \\
\hline 17 & 6 & 0 & 6.226996 & 1.863758 & -2.270926 \\
\hline 18 & 6 & 0 & 2.607106 & -0.796637 & -0.005537 \\
\hline 19 & 8 & 0 & 0.077649 & 1. 122123 & -0.037907 \\
\hline 20 & 6 & 0 & -0.992807 & 2.025144 & 0.097736 \\
\hline 21 & 6 & 0 & -2.197059 & 1.543825 & -0.676924 \\
\hline 22 & 6 & 0 & -3.470722 & 1.801125 & -0.265619 \\
\hline 23 & 1 & 0 & -3.598446 & 2. 139588 & 0.763410 \\
\hline 24 & 53 & 0 & -1.289447 & -2.893729 & 1. 957063 \\
\hline 25 & 6 & 0 & -4.708622 & 1.684203 & -1.028841 \\
\hline 26 & 8 & 0 & 5.260267 & -0.222370 & -0.788310 \\
\hline 27 & 6 & 0 & 3.923046 & 2.721001 & -2.443981 \\
\hline 28 & 6 & 0 & 4. 190025 & -3.146349 & -1.008580 \\
\hline 29 & 1 & 0 & 3. 490001 & -1.113534 & 0.551218 \\
\hline 30 & 1 & 0 & 2.387723 & 0.396487 & -1.834453 \\
\hline 31 & 1 & 0 & -1.260763 & 2. 184633 & 1. 152292 \\
\hline
\end{tabular}




\begin{tabular}{|c|c|c|c|c|c|}
\hline 32 & 1 & 0 & -0.700199 & 3. 005268 & -0.312637 \\
\hline 33 & 1 & 0 & -2.936996 & -0.429306 & 0.999365 \\
\hline 34 & 1 & 0 & -1.996161 & 1. 246366 & -1.704837 \\
\hline 35 & 1 & 0 & 3.838667 & 2. 656090 & -3.538004 \\
\hline 36 & 1 & 0 & 4. 330009 & 3.708585 & -2.193678 \\
\hline 37 & 1 & 0 & 2. 941837 & 2. 641617 & -1.982542 \\
\hline 38 & 1 & 0 & 6. 791687 & 1. 031463 & -1.854015 \\
\hline 39 & 1 & 0 & 6.603603 & 2. 807704 & -1.856592 \\
\hline 40 & 1 & 0 & 6. 356646 & 1. 889315 & -3.360985 \\
\hline 41 & 1 & 0 & 1. 775047 & 6.165545 & 4. 303460 \\
\hline 42 & 1 & 0 & 0.892907 & 6. 148652 & 2. 768139 \\
\hline 43 & 1 & 0 & 2. 652069 & 5.962270 & 2.778410 \\
\hline 44 & 1 & 0 & 2. 406565 & 3.755458 & 3. 992219 \\
\hline 45 & 1 & 0 & 0.661472 & 3. 940611 & 3. 987890 \\
\hline 46 & 1 & 0 & 0.672072 & 3.910864 & 1. 475449 \\
\hline 47 & 1 & 0 & 2. 412881 & 3.708353 & 1. 479433 \\
\hline 48 & 1 & 0 & 2. 181086 & 1.553547 & 2.752175 \\
\hline 49 & 1 & 0 & 0.441199 & 1. 698987 & 2. 711388 \\
\hline 50 & 1 & 0 & 4. 677037 & -2.214277 & -0.726356 \\
\hline 51 & 1 & 0 & 4. 680129 & -3.522944 & -1.912468 \\
\hline 52 & 1 & 0 & 4. 303140 & -3.897023 & -0.216486 \\
\hline 53 & 1 & 0 & 1. 067403 & -3.869595 & -2.106757 \\
\hline 54 & 1 & 0 & 2. 354882 & -4.984101 & -1.572744 \\
\hline 55 & 1 & 0 & 2. 552986 & -4.060643 & -3.082621 \\
\hline 56 & 6 & 0 & -5.938475 & 1.815898 & -0.351660 \\
\hline 57 & 6 & 0 & -7.149082 & 1. 716911 & -1.031830 \\
\hline 58 & 6 & 0 & -7.160651 & 1. 485743 & -2.409693 \\
\hline 59 & 6 & 0 & -5.951553 & 1. 356948 & -3.099869 \\
\hline 60 & 6 & 0 & -4.740105 & 1. 456289 & -2.421134 \\
\hline 61 & 1 & 0 & -5.934140 & 1.995764 & 0.720738 \\
\hline 62 & 1 & 0 & -8.083840 & 1.820293 & -0.487929 \\
\hline 63 & 1 & 0 & -8.103974 & 1. 411063 & -2.943274 \\
\hline 64 & 1 & 0 & -5.953586 & 1. 185516 & -4.172792 \\
\hline 65 & 1 & 0 & -3.810020 & 1. 375527 & -2.975836 \\
\hline 66 & 6 & 0 & -3.273851 & -1.461864 & -0.871956 \\
\hline 67 & 1 & 0 & -4.121262 & -0.842506 & -1.198474 \\
\hline 68 & 1 & 0 & -2.733148 & -1.810275 & -1.757297 \\
\hline 69 & 1 & 0 & -3.717491 & -2.331966 & -0.372607 \\
\hline
\end{tabular}




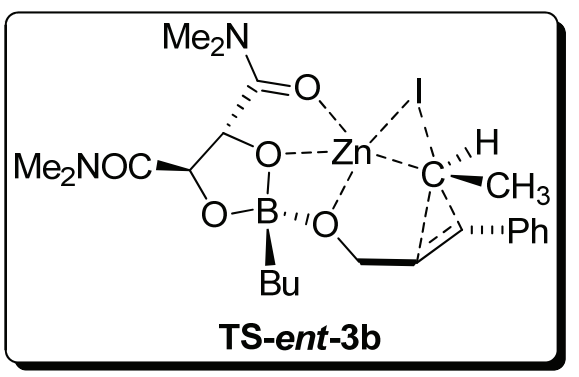

Imaginary frequency: $-161.6 \mathrm{~cm}^{-1}$

Standard orientation:

\begin{tabular}{|c|c|c|c|c|c|}
\hline \multirow{2}{*}{$\begin{array}{l}\text { Center } \\
\text { Number }\end{array}$} & \multirow{2}{*}{$\begin{array}{l}\text { Atomic } \\
\text { Number }\end{array}$} & \multirow{2}{*}{$\begin{array}{l}\text { Atomic } \\
\text { Type }\end{array}$} & \multicolumn{2}{|c|}{ Coordinates } & \multirow{2}{*}{$\begin{array}{r}(\text { Angstroms) } \\
Z\end{array}$} \\
\hline & & & $X$ & Y & \\
\hline 1 & 6 & 0 & 4. 833157 & 2. 790397 & 1. 210182 \\
\hline 2 & 6 & 0 & 3.893686 & 1. 818846 & 1. 608932 \\
\hline 3 & 6 & 0 & 4. 296799 & 0.850373 & 2.547401 \\
\hline 4 & 6 & 0 & 5.590788 & 0.844940 & 3.064840 \\
\hline 5 & 6 & 0 & 6.510785 & 1. 811284 & 2. 654744 \\
\hline 6 & 6 & 0 & 6.125354 & 2. 783992 & 1. 726294 \\
\hline 7 & 6 & 0 & 2. 518386 & 1. 768680 & 1. 102584 \\
\hline 8 & 6 & 0 & 1.967915 & 2. 540666 & 0.131709 \\
\hline 9 & 6 & 0 & 0.504431 & 2. 568147 & -0.228439 \\
\hline 10 & 8 & 0 & -0.143157 & 1. 393519 & 0.164725 \\
\hline 11 & 5 & 0 & -1.534570 & 1.000193 & -0.249174 \\
\hline 12 & 8 & 0 & -2.400302 & 0.909144 & 0.931483 \\
\hline 13 & 6 & 0 & -2.775033 & -0.394857 & 1. 247841 \\
\hline 14 & 6 & 0 & -1.886887 & -1.333949 & 0.354632 \\
\hline 15 & 8 & 0 & -1.325861 & -0.484543 & -0.623457 \\
\hline 16 & 30 & 0 & 0.747894 & -0.530229 & -0.689816 \\
\hline 17 & 53 & 0 & 1. 727231 & -2.499062 & -2.355750 \\
\hline 18 & 6 & 0 & 2. 478060 & 0.413449 & -1.136691 \\
\hline 19 & 8 & 0 & 0.414524 & -1.586048 & 1. 047098 \\
\hline 20 & 6 & 0 & -0.764192 & -2.003690 & 1. 167333 \\
\hline 21 & 7 & 0 & -1.048161 & -3.020314 & 1. 999881 \\
\hline 22 & 6 & 0 & -2.370266 & -3.621527 & 2. 172796 \\
\hline 23 & 6 & 0 & 0.023584 & -3.662909 & 2.759357 \\
\hline 24 & 6 & 0 & -2.173224 & 1.814036 & -1.488056 \\
\hline 25 & 6 & 0 & -2.747458 & 3. 207168 & -1.172563 \\
\hline 26 & 6 & 0 & -3.442754 & 3. 877361 & -2.366532 \\
\hline 27 & 6 & 0 & -4.025915 & 5.256720 & -2.040446 \\
\hline 28 & 6 & 0 & -4.283220 & -0.710718 & 1. 027841 \\
\hline 29 & 8 & 0 & -4.606857 & -1.807214 & 0.555284 \\
\hline 30 & 7 & 0 & -5.213019 & 0.206123 & 1. 416445 \\
\hline 31 & 6 & 0 & -4.957684 & 1. 441810 & 2. 148280 \\
\hline
\end{tabular}




\begin{tabular}{|c|c|c|c|c|c|}
\hline 32 & 6 & 0 & -6.623964 & -0.103993 & 1. 215300 \\
\hline 33 & 1 & 0 & 1. 865435 & 1. 039415 & 1. 577565 \\
\hline 34 & 1 & 0 & -2.507799 & -2.088708 & -0.131495 \\
\hline 35 & 1 & 0 & -2.578722 & -0.590237 & 2. 316323 \\
\hline 36 & 1 & 0 & 0.407333 & 2. 753080 & -1.310130 \\
\hline 37 & 1 & 0 & 0.062477 & 3. 450489 & 0.267607 \\
\hline 38 & 6 & 0 & 2. 915505 & 1. 161914 & -2.356067 \\
\hline 39 & 1 & 0 & 3. 347688 & 0.020969 & -0.597024 \\
\hline 40 & 1 & 0 & 2. 567219 & 3. 307242 & -0.357324 \\
\hline 41 & 1 & 0 & -5.426228 & 1. 388153 & 3.141089 \\
\hline 42 & 1 & 0 & -5.401411 & 2. 288774 & 1. 609546 \\
\hline 43 & 1 & 0 & -3.891924 & 1. 626190 & 2. 244349 \\
\hline 44 & 1 & 0 & -6.705256 & -1.015473 & 0.625747 \\
\hline 45 & 1 & 0 & -7.114493 & 0.725514 & 0.691600 \\
\hline 46 & 1 & 0 & -7.128536 & -0.249499 & 2. 180316 \\
\hline 47 & 1 & 0 & -4.516880 & 5.704443 & -2.912736 \\
\hline 48 & 1 & 0 & -3.243423 & 5. 950634 & -1.707343 \\
\hline 49 & 1 & 0 & -4.770446 & 5. 192326 & -1.236659 \\
\hline 50 & 1 & 0 & -4.242802 & 3. 217429 & -2.731545 \\
\hline 51 & 1 & 0 & -2.727445 & 3. 969244 & -3.196913 \\
\hline 52 & 1 & 0 & -1.956733 & 3.880744 & -0.807972 \\
\hline 53 & 1 & 0 & -3.463723 & 3. 118747 & -0.343313 \\
\hline 54 & 1 & 0 & -2.989670 & 1. 190745 & -1.883574 \\
\hline 55 & 1 & 0 & -1.457539 & 1. 897968 & -2.323239 \\
\hline 56 & 1 & 0 & -3.136991 & -3.115868 & 1.586448 \\
\hline 57 & 1 & 0 & -2.652510 & -3.582290 & 3.232156 \\
\hline 58 & 1 & 0 & -2.332226 & -4.674407 & 1. 867892 \\
\hline 59 & 1 & 0 & 0.966377 & -3.165697 & 2.543205 \\
\hline 60 & 1 & 0 & 0.094116 & -4.719363 & 2. 475611 \\
\hline 61 & 1 & 0 & -0.194145 & -3.601445 & 3.832190 \\
\hline 62 & 1 & 0 & 3.582403 & 0.095176 & 2.866005 \\
\hline 63 & 1 & 0 & 5.880504 & 0.086422 & 3.786665 \\
\hline 64 & 1 & 0 & 7. 520154 & 1.810916 & 3.056501 \\
\hline 65 & 1 & 0 & 6.835065 & 3. 542866 & 1. 408361 \\
\hline 66 & 1 & 0 & 4. 549269 & 3. 562969 & 0.501451 \\
\hline 67 & 1 & 0 & 3. 819992 & 1. 764140 & -2.201113 \\
\hline 68 & 1 & 0 & 3. 181967 & 0.391981 & -3.095442 \\
\hline 69 & 1 & 0 & 2. 126034 & 1. 774224 & -2.802685 \\
\hline
\end{tabular}




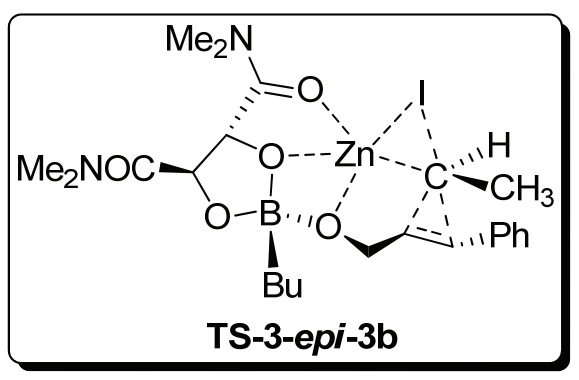

Imaginary frequency: $-259.4 \mathrm{~cm}^{-1}$

Standard orientation:

\begin{tabular}{|c|c|c|c|c|c|}
\hline \multirow{2}{*}{$\begin{array}{l}\text { Center } \\
\text { Number }\end{array}$} & \multirow{2}{*}{$\begin{array}{l}\text { Atomic } \\
\text { Number }\end{array}$} & \multirow{2}{*}{$\begin{array}{l}\text { Atomic } \\
\text { Type }\end{array}$} & \multicolumn{2}{|c|}{ Coordinates } & (Angstroms) \\
\hline & & & X & Y & Z \\
\hline 1 & 6 & 0 & 2.351747 & -0.014735 & -0.731471 \\
\hline 2 & 30 & 0 & 0.531422 & -0.726897 & -0.224179 \\
\hline 3 & 8 & 0 & -0.382511 & -1.928009 & 1. 136394 \\
\hline 4 & 6 & 0 & -1.611945 & -2.104722 & 0.939932 \\
\hline 5 & 7 & 0 & -2.248191 & -3.138355 & 1. 515788 \\
\hline 6 & 6 & 0 & -1.505259 & -4.073443 & 2. 361067 \\
\hline 7 & 8 & 0 & -1.471627 & -0.308165 & -0.669804 \\
\hline 8 & 5 & 0 & -1.471158 & 1. 131896 & -0.107945 \\
\hline 9 & 6 & 0 & -1.574528 & 2.228846 & -1.284699 \\
\hline 10 & 6 & 0 & -1.953593 & 3.653844 & -0.841392 \\
\hline 11 & 6 & 0 & -2.127849 & 4.642551 & -2.003046 \\
\hline 12 & 6 & 0 & -2.523892 & 6.052958 & -1.552514 \\
\hline 13 & 8 & 0 & -2.619008 & 1. 119443 & 0.800828 \\
\hline 14 & 6 & 0 & -3.253591 & -0.119402 & 0.885190 \\
\hline 15 & 6 & 0 & -4.721053 & -0.108726 & 0.368432 \\
\hline 16 & 7 & 0 & -5.539488 & 0.912516 & 0.746142 \\
\hline 17 & 6 & 0 & -6.915063 & 0.921497 & 0.261732 \\
\hline 18 & 6 & 0 & -2.382174 & -1.119595 & 0.041359 \\
\hline 19 & 8 & 0 & -0.176075 & 1. 118388 & 0.670852 \\
\hline 20 & 6 & 0 & 0.777765 & 2. 152971 & 0.726660 \\
\hline 21 & 6 & 0 & 2. 135910 & 1.574098 & 1. 046672 \\
\hline 22 & 6 & 0 & 3.310315 & 2.133424 & 0.649270 \\
\hline 23 & 1 & 0 & 3.257652 & 2.925898 & -0.097457 \\
\hline 24 & 53 & 0 & 1. 771515 & -2.770486 & -1.783900 \\
\hline 25 & 6 & 0 & 4. 663556 & 1.794406 & 1.087165 \\
\hline 26 & 8 & 0 & -5.126321 & -1.044945 & -0.331247 \\
\hline 27 & 6 & 0 & -5.219086 & 1.971238 & 1. 696853 \\
\hline 28 & 6 & 0 & -3.662296 & -3.464714 & 1.333911 \\
\hline 29 & 1 & 0 & -3.014172 & -1.661822 & -0.664578 \\
\hline 30 & 1 & 0 & -3.299355 & -0.450169 & 1. 937508 \\
\hline 31 & 1 & 0 & 0.822915 & 2. 727600 & -0.208540 \\
\hline
\end{tabular}




\begin{tabular}{|c|c|c|c|c|c|}
\hline 32 & 1 & 0 & 0.505475 & 2.860307 & 1. 527381 \\
\hline 33 & 1 & 0 & 3. 238704 & -0.467633 & -0.287148 \\
\hline 34 & 1 & 0 & 2. 128834 & 0.814842 & 1. 826711 \\
\hline 35 & 1 & 0 & -5.868337 & 1. 886431 & 2. 579590 \\
\hline 36 & 1 & 0 & -5.401235 & 2.950026 & 1.235114 \\
\hline 37 & 1 & 0 & -4.175088 & 1. 929745 & 1. 992396 \\
\hline 38 & 1 & 0 & -7.038934 & 0.120753 & -0.465183 \\
\hline 39 & 1 & 0 & -7.137282 & 1. 888384 & -0.206130 \\
\hline 40 & 1 & 0 & -7.617379 & 0.770874 & 1. 092934 \\
\hline 41 & 1 & 0 & -2.639343 & 6. 732039 & -2.405686 \\
\hline 42 & 1 & 0 & -1.766904 & 6. 483967 & -0.884759 \\
\hline 43 & 1 & 0 & -3.475180 & 6.041726 & -1.005493 \\
\hline 44 & 1 & 0 & -2.887003 & 4. 251590 & -2.695686 \\
\hline 45 & 1 & 0 & -1.192694 & 4. 692279 & -2.580231 \\
\hline 46 & 1 & 0 & -1.199617 & 4. 057802 & -0.147881 \\
\hline 47 & 1 & 0 & -2.887593 & 3. 607721 & -0.263985 \\
\hline 48 & 1 & 0 & -2.343212 & 1.872610 & -1.987016 \\
\hline 49 & 1 & 0 & -0.647112 & 2. 268471 & -1.880959 \\
\hline 50 & 1 & 0 & -4.177850 & -2.743647 & 0.700313 \\
\hline 51 & 1 & 0 & -4.157870 & -3.492554 & 2. 312224 \\
\hline 52 & 1 & 0 & -3.748299 & -4.458773 & 0.878755 \\
\hline 53 & 1 & 0 & -0.453764 & -3.795960 & 2. 369451 \\
\hline 54 & 1 & 0 & -1.613908 & -5.088791 & 1.963595 \\
\hline 55 & 1 & 0 & -1.904763 & -4.051346 & 3. 382044 \\
\hline 56 & 6 & 0 & 5. 753927 & 2. 418290 & 0.449994 \\
\hline 57 & 6 & 0 & 7. 065980 & 2. 127168 & 0.817656 \\
\hline 58 & 6 & 0 & 7. 317552 & 1.204013 & 1.833900 \\
\hline 59 & 6 & 0 & 6. 246302 & 0.578633 & 2.481374 \\
\hline 60 & 6 & 0 & 4. 936110 & 0.870574 & 2. 117061 \\
\hline 61 & 1 & 0 & 5.562315 & 3.139077 & -0.341566 \\
\hline 62 & 1 & 0 & 7. 890836 & 2.620034 & 0.310592 \\
\hline 63 & 1 & 0 & 8. 339033 & 0.974025 & 2. 123442 \\
\hline 64 & 1 & 0 & 6.435589 & -0.137233 & 3.276498 \\
\hline 65 & 1 & 0 & 4. 118129 & 0.382930 & 2.639077 \\
\hline 66 & 6 & 0 & 2. 641850 & 0.756360 & -1.995835 \\
\hline 67 & 1 & 0 & 3.683097 & 1. 081920 & -2.093325 \\
\hline 68 & 1 & 0 & 2. 424694 & 0.101671 & -2.846571 \\
\hline 69 & 1 & 0 & 1. 976418 & 1. 622341 & -2.098981 \\
\hline
\end{tabular}




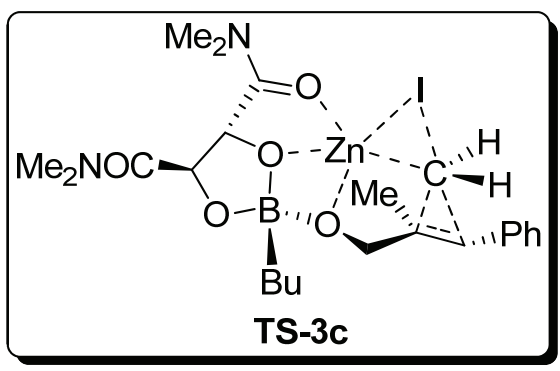

Imaginary frequency: $-336.8 \mathrm{~cm}^{-1}$

Standard orientation:

\begin{tabular}{|c|c|c|c|c|c|}
\hline \multirow{2}{*}{$\begin{array}{l}\text { Center } \\
\text { Number }\end{array}$} & \multirow{2}{*}{$\begin{array}{l}\text { Atomic } \\
\text { Number }\end{array}$} & \multirow{2}{*}{$\begin{array}{l}\text { Atomic } \\
\text { Type }\end{array}$} & \multicolumn{2}{|c|}{ Coordinates } & \multirow{2}{*}{$\begin{array}{r}(\text { Angstroms) } \\
Z\end{array}$} \\
\hline & & & $X$ & Y & \\
\hline 1 & 6 & 0 & 2. 364956 & -0.380639 & -0.399899 \\
\hline 2 & 30 & 0 & 0.456314 & -0.867646 & -0.065381 \\
\hline 3 & 8 & 0 & -0.600901 & -2.053983 & 1. 185045 \\
\hline 4 & 6 & 0 & -1.828252 & -2.146363 & 0.918244 \\
\hline 5 & 7 & 0 & -2.561282 & -3.142418 & 1. 439097 \\
\hline 6 & 6 & 0 & -1.930897 & -4.140765 & 2. 303689 \\
\hline 7 & 8 & 0 & -1.477764 & -0.352594 & -0.653483 \\
\hline 8 & 5 & 0 & -1.435135 & 1. 104521 & -0.119135 \\
\hline 9 & 6 & 0 & -1.577931 & 2. 166837 & -1.321218 \\
\hline 10 & 6 & 0 & -1.799984 & 3.632636 & -0.905953 \\
\hline 11 & 6 & 0 & -2.026788 & 4. 587757 & -2.086554 \\
\hline 12 & 6 & 0 & -2.257872 & 6. 041992 & -1.661219 \\
\hline 13 & 8 & 0 & -2.537085 & 1. 109779 & 0.849888 \\
\hline 14 & 6 & 0 & -3.312392 & -0.046000 & 0.814843 \\
\hline 15 & 6 & 0 & -4.728338 & 0.117264 & 0.187814 \\
\hline 16 & 7 & 0 & -5.460697 & 1. 227103 & 0.482086 \\
\hline 17 & 6 & 0 & -6.790207 & 1. 359919 & -0.103343 \\
\hline 18 & 6 & 0 & -2.480149 & -1.100543 & -0.002578 \\
\hline 19 & 8 & 0 & -0.125412 & 1. 103143 & 0.625383 \\
\hline 20 & 6 & 0 & 0.921336 & 2. 037479 & 0.477737 \\
\hline 21 & 6 & 0 & 2. 246342 & 1. 452529 & 0.959232 \\
\hline 22 & 6 & 0 & 3. 391768 & 1. 815288 & 0.293000 \\
\hline 23 & 1 & 0 & 3. 236203 & 2. 298632 & -0.671667 \\
\hline 24 & 53 & 0 & 1. 780403 & -2.777401 & -1.791071 \\
\hline 25 & 6 & 0 & 4. 807532 & 1. 663959 & 0.642463 \\
\hline 26 & 8 & 0 & -5.178162 & -0.792758 & -0.519805 \\
\hline 27 & 6 & 0 & -5.115768 & 2. 260024 & 1. 453234 \\
\hline 28 & 6 & 0 & -3.984576 & -3.362369 & 1. 179146 \\
\hline 29 & 1 & 0 & -3.115766 & -1.588248 & -0.743737 \\
\hline 30 & 1 & 0 & -3.480846 & -0.407558 & 1.843695 \\
\hline 31 & 1 & 0 & 1. 019796 & 2.377755 & -0.560739 \\
\hline
\end{tabular}




\begin{tabular}{|c|c|c|c|c|c|}
\hline 32 & 1 & 0 & 0.705637 & 2. 924303 & 1. 095332 \\
\hline 33 & 1 & 0 & 2. 632035 & 0.071133 & -1.353815 \\
\hline 34 & 1 & 0 & 3. 230377 & -0.842775 & 0.071785 \\
\hline 35 & 1 & 0 & -5.842417 & 2. 250553 & 2. 277824 \\
\hline 36 & 1 & 0 & -5.159470 & 3.246563 & 0.974657 \\
\hline 37 & 1 & 0 & -4.110151 & 2. 115799 & 1. 836488 \\
\hline 38 & 1 & 0 & -6.932479 & 0.572681 & -0.841488 \\
\hline 39 & 1 & 0 & -6.887308 & 2. 342678 & -0.580303 \\
\hline 40 & 1 & 0 & -7.563674 & 1. 274497 & 0.672172 \\
\hline 41 & 1 & 0 & -2.416096 & 6.695836 & -2.527119 \\
\hline 42 & 1 & 0 & -1.398359 & 6.433467 & -1.102089 \\
\hline 43 & 1 & 0 & -3.138663 & 6.130573 & -1.012435 \\
\hline 44 & 1 & 0 & -2.887337 & 4. 235461 & -2.673411 \\
\hline 45 & 1 & 0 & -1.161504 & 4. 537916 & -2.763655 \\
\hline 46 & 1 & 0 & -0.945088 & 4. 001527 & -0.318808 \\
\hline 47 & 1 & 0 & -2.665204 & 3. 686177 & -0.229671 \\
\hline 48 & 1 & 0 & -2.439719 & 1.847559 & -1.927584 \\
\hline 49 & 1 & 0 & -0.717910 & 2. 109759 & -2.009012 \\
\hline 50 & 1 & 0 & -4.418123 & -2.584246 & 0.551193 \\
\hline 51 & 1 & 0 & -4.526113 & -3.388916 & 2. 132538 \\
\hline 52 & 1 & 0 & -4.116949 & -4.331393 & 0.683094 \\
\hline 53 & 1 & 0 & -0.862892 & -3.944289 & 2. 364287 \\
\hline 54 & 1 & 0 & -2.098144 & -5.140139 & 1.886726 \\
\hline 55 & 1 & 0 & -2.374024 & -4.099857 & 3.305780 \\
\hline 56 & 6 & 0 & 5. 746141 & 1. 697323 & -0.411238 \\
\hline 57 & 6 & 0 & 7. 110401 & 1. 568968 & -0.171529 \\
\hline 58 & 6 & 0 & 7. 579538 & 1. 420583 & 1.136360 \\
\hline 59 & 6 & 0 & 6.669944 & 1. 411122 & 2. 195623 \\
\hline 60 & 6 & 0 & 5.301954 & 1. 531767 & 1. 956540 \\
\hline 61 & 1 & 0 & 5. 388683 & 1.813729 & -1.431577 \\
\hline 62 & 1 & 0 & 7. 808306 & 1.587008 & -1.004020 \\
\hline 63 & 1 & 0 & 8. 644391 & 1. 323149 & 1. 328400 \\
\hline 64 & 1 & 0 & 7. 026864 & 1. 316707 & 3.217694 \\
\hline 65 & 1 & 0 & 4. 622987 & 1. 554836 & 2. 799648 \\
\hline 66 & 6 & 0 & 2. 200919 & 0.833045 & 2. 337722 \\
\hline 67 & 1 & 0 & 2. 999448 & 0.104430 & 2. 494955 \\
\hline 68 & 1 & 0 & 2. 292468 & 1. 611329 & 3.108158 \\
\hline 69 & 1 & 0 & 1. 238350 & 0.340048 & 2. 493345 \\
\hline
\end{tabular}




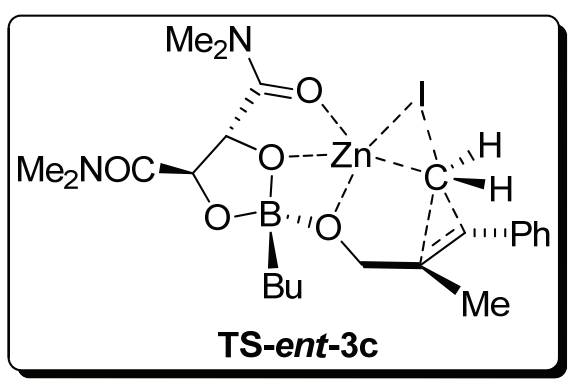

Imaginary frequency: $-206.2 \mathrm{~cm}^{-1}$

Standard orientation:

\begin{tabular}{|c|c|c|c|c|c|}
\hline \multirow{2}{*}{$\begin{array}{l}\text { Center } \\
\text { Number }\end{array}$} & \multirow{2}{*}{$\begin{array}{l}\text { Atomic } \\
\text { Number }\end{array}$} & \multirow{2}{*}{$\begin{array}{l}\text { Atomic } \\
\text { Type }\end{array}$} & \multicolumn{2}{|c|}{ Coordinates } & $($ Angstroms $)$ \\
\hline & & & X & Y & Z \\
\hline 1 & 6 & 0 & 2. 494673 & 0.029716 & -0.798789 \\
\hline 2 & 30 & 0 & 0.762061 & -0.854733 & -0.425803 \\
\hline 3 & 8 & 0 & 0.132503 & -1.993387 & 1. 143145 \\
\hline 4 & 6 & 0 & -1.097269 & -2.255901 & 1. 097657 \\
\hline 5 & 7 & 0 & -1.600687 & -3.295750 & 1. 781269 \\
\hline 6 & 6 & 0 & -0.712112 & -4.156362 & 2. 562379 \\
\hline 7 & 8 & 0 & -1.235526 & -0.552619 & -0.594606 \\
\hline 8 & 5 & 0 & -1.433437 & 0.978495 & -0.250360 \\
\hline 9 & 6 & 0 & -2.248117 & 1. 689278 & -1.450289 \\
\hline 10 & 6 & 0 & -2.722819 & 3.127723 & -1.174542 \\
\hline 11 & 6 & 0 & -3.584388 & 3.727567 & -2.295014 \\
\hline 12 & 6 & 0 & -4.054922 & 5. 157411 & -2.005654 \\
\hline 13 & 8 & 0 & -2.152345 & 0.876500 & 1. 028806 \\
\hline 14 & 6 & 0 & -2.801290 & -0.344071 & 1. 175978 \\
\hline 15 & 6 & 0 & -4.318273 & -0.368099 & 0.821862 \\
\hline 16 & 7 & 0 & -5.108048 & 0.660329 & 1. 239558 \\
\hline 17 & 6 & 0 & -6.533089 & 0.613273 & 0.931669 \\
\hline 18 & 6 & 0 & -2.017998 & -1.357870 & 0.260298 \\
\hline 19 & 8 & 0 & -0.077667 & 1. 472532 & 0.045895 \\
\hline 20 & 6 & 0 & 0.654045 & 2. 272392 & -0.834613 \\
\hline 21 & 6 & 0 & 2. 133280 & 2. 322186 & -0.487080 \\
\hline 22 & 6 & 0 & 2. 570025 & 1. 846000 & 0.720580 \\
\hline 23 & 1 & 0 & 1. 796889 & 1. 377906 & 1. 323449 \\
\hline 24 & 53 & 0 & 2. 056178 & -2.653417 & -2.131726 \\
\hline 25 & 6 & 0 & 3.888475 & 1. 942497 & 1. 368154 \\
\hline 26 & 8 & 0 & -4.781584 & -1.345765 & 0.221887 \\
\hline 27 & 6 & 0 & -4.708163 & 1. 740512 & 2. 135471 \\
\hline 28 & 6 & 0 & -3.005540 & -3.704587 & 1. 770021 \\
\hline 29 & 1 & 0 & -2.717905 & -1.954032 & -0.327571 \\
\hline 30 & 1 & 0 & -2.739210 & -0.655948 & 2. 232005 \\
\hline
\end{tabular}




\begin{tabular}{|c|c|c|c|c|c|}
\hline 31 & 1 & 0 & 0.564036 & 1. 933367 & -1.878774 \\
\hline 32 & 1 & 0 & 0.291985 & 3. 315567 & -0.824758 \\
\hline 33 & 1 & 0 & 2. 723626 & 0.417791 & -1.793340 \\
\hline 34 & 1 & 0 & 3. 426265 & -0.261449 & -0.308955 \\
\hline 35 & 1 & 0 & -5.199183 & 1. 621272 & 3. 112016 \\
\hline 36 & 1 & 0 & -5.025507 & 2. 701543 & 1. 712585 \\
\hline 37 & 1 & 0 & -3.628707 & 1. 763914 & 2. 255055 \\
\hline 38 & 1 & 0 & -6.715182 & -0.188874 & 0.218599 \\
\hline 39 & 1 & 0 & -6.849357 & 1. 572653 & 0.505396 \\
\hline 40 & 1 & 0 & -7.121188 & 0.428604 & 1. 841365 \\
\hline 41 & 1 & 0 & -4.669329 & 5. 554743 & -2.822351 \\
\hline 42 & 1 & 0 & -3.202699 & 5.835748 & -1.870534 \\
\hline 43 & 1 & 0 & -4.654843 & 5. 199299 & -1.087400 \\
\hline 44 & 1 & 0 & -4.457425 & 3.080095 & -2.461165 \\
\hline 45 & 1 & 0 & -3.016051 & 3. 712181 & -3.236347 \\
\hline 46 & 1 & 0 & -1.861102 & 3. 791152 & -1.003349 \\
\hline 47 & 1 & 0 & -3.294177 & 3.144232 & -0.235542 \\
\hline 48 & 1 & 0 & -3.134856 & 1. 070726 & -1.661676 \\
\hline 49 & 1 & 0 & -1.671993 & 1. 671398 & -2.389828 \\
\hline 50 & 1 & 0 & -3.635196 & -3.011163 & 1. 213008 \\
\hline 51 & 1 & 0 & -3.372610 & -3.761928 & 2.801824 \\
\hline 52 & 1 & 0 & -3.092099 & -4.700970 & 1. 319810 \\
\hline 53 & 1 & 0 & 0.312975 & -3.809252 & 2. 454013 \\
\hline 54 & 1 & 0 & -0.790704 & -5.187566 & 2. 199770 \\
\hline 55 & 1 & 0 & -1.004009 & -4.132083 & 3.618876 \\
\hline 56 & 6 & 0 & 4. 213246 & 0.967329 & 2. 332832 \\
\hline 57 & 6 & 0 & 5. 434689 & 0.991504 & 2. 999848 \\
\hline 58 & 6 & 0 & 6.355292 & 2. 008899 & 2.735703 \\
\hline 59 & 6 & 0 & 6.037778 & 3. 001399 & 1. 806771 \\
\hline 60 & 6 & 0 & 4. 819243 & 2. 971215 & 1. 129039 \\
\hline 61 & 1 & 0 & 3. 496104 & 0.178925 & 2. 548188 \\
\hline 62 & 1 & 0 & 5.666330 & 0.221036 & 3. 729955 \\
\hline 63 & 1 & 0 & 7. 307236 & 2. 034361 & 3.258638 \\
\hline 64 & 1 & 0 & 6. 738171 & 3. 809094 & 1. 612434 \\
\hline 65 & 1 & 0 & 4. 580543 & 3. 772589 & 0.440012 \\
\hline 66 & 6 & 0 & 2.972137 & 3. 074632 & -1.490181 \\
\hline 67 & 1 & 0 & 2. 931562 & 4. 154947 & -1.294421 \\
\hline 68 & 1 & 0 & 4. 020590 & 2. 769244 & -1.467490 \\
\hline 69 & 1 & 0 & 2. 589632 & 2. 921485 & -2.505515 \\
\hline
\end{tabular}




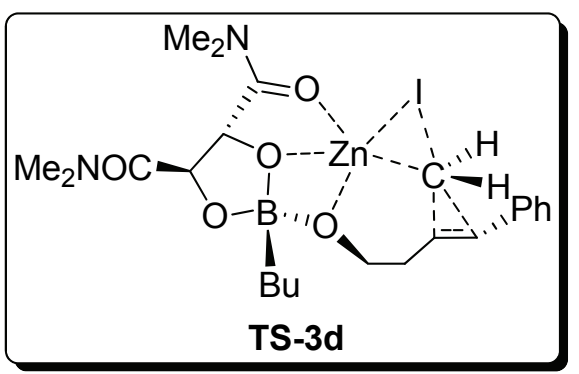

Imaginary frequency: $-330.0 \mathrm{~cm}^{-1}$

Standard orientation:

\begin{tabular}{|c|c|c|c|c|c|}
\hline \multirow{3}{*}{$\begin{array}{c}\text { Center } \\
\text { Number } \\
------- \\
1\end{array}$} & \multirow{3}{*}{$\begin{array}{c}\text { Atomic } \\
\text { Number } \\
------- \\
6\end{array}$} & \multirow{3}{*}{$\begin{array}{c}\text { Atomic } \\
\text { Type } \\
0\end{array}$} & \multicolumn{2}{|c|}{ Coordinates } & \multirow{3}{*}{$\begin{array}{r}(\text { Angstroms }) \\
Z \\
0.565007\end{array}$} \\
\hline & & & $X$ & Y & \\
\hline & & & 2. 439992 & -0.900555 & \\
\hline 2 & 30 & 0 & 0.610917 & -0.092565 & 0.626208 \\
\hline 3 & 8 & 0 & 0.495824 & 1.805891 & -0.166994 \\
\hline 4 & 6 & 0 & -0.599044 & 2. 407102 & -0.024190 \\
\hline 5 & 7 & 0 & -0.681724 & 3.734042 & -0.218102 \\
\hline 6 & 6 & 0 & 0.518180 & 4. 499024 & -0.555993 \\
\hline 7 & 8 & 0 & -1.463035 & 0.344751 & 0.860835 \\
\hline 8 & 5 & 0 & -1.925011 & -0.782796 & -0.075536 \\
\hline 9 & 6 & 0 & -2.982268 & -1.754982 & 0.648896 \\
\hline 10 & 6 & 0 & -3.371267 & -3.024470 & -0.128116 \\
\hline 11 & 6 & 0 & -4.444597 & -3.877781 & 0.563506 \\
\hline 12 & 6 & 0 & -4.803343 & -5.153177 & -0.207046 \\
\hline 13 & 8 & 0 & -2.403828 & -0.040748 & -1.255480 \\
\hline 14 & 6 & 0 & -2.716856 & 1. 276322 & -0.925387 \\
\hline 15 & 6 & 0 & -4.212425 & 1.601548 & -0.637254 \\
\hline 16 & 7 & 0 & -5.198321 & 0.956655 & -1.319564 \\
\hline 17 & 6 & 0 & -6.584960 & 1. 296974 & -1.016912 \\
\hline 18 & 6 & 0 & -1.849880 & 1.599947 & 0.351102 \\
\hline 19 & 8 & 0 & -0.606472 & -1.455853 & -0.377282 \\
\hline 20 & 6 & 0 & -0.298661 & -1.997038 & -1.651566 \\
\hline 21 & 6 & 0 & 2. 226714 & -1.458318 & -1.674055 \\
\hline 22 & 6 & 0 & 3.553544 & -1.762556 & -1.581070 \\
\hline 23 & 1 & 0 & 3.814478 & -2.787718 & -1.316328 \\
\hline 24 & 53 & 0 & 1.904587 & -0.099666 & 3.174356 \\
\hline 25 & 6 & 0 & 4. 695270 & -0.880375 & -1.830415 \\
\hline 26 & 8 & 0 & -4.464752 & 2. 494335 & 0.182013 \\
\hline 27 & 6 & 0 & -5.028368 & 0.062910 & -2.460841 \\
\hline 28 & 6 & 0 & -1.897036 & 4. 533161 & -0.051071 \\
\hline 29 & 1 & 0 & -2.447930 & 2. 132213 & 1. 091691 \\
\hline 30 & 1 & 0 & -2.432650 & 1.934960 & -1.762194 \\
\hline 31 & 1 & 0 & -0.979348 & -2.830718 & -1.8652 \\
\hline
\end{tabular}




\begin{tabular}{|c|c|c|c|c|c|}
\hline 32 & 1 & 0 & -0.455129 & -1.238747 & -2.432205 \\
\hline 33 & 1 & 0 & 2. 626336 & -1.917881 & 0.905874 \\
\hline 34 & 1 & 0 & 3. 356685 & -0.315210 & 0.532648 \\
\hline 35 & 1 & 0 & 1.934485 & -0.471798 & -2.030065 \\
\hline 36 & 1 & 0 & -5.485342 & 0.514308 & -3.352662 \\
\hline 37 & 1 & 0 & -5.535915 & -0.889660 & -2.263827 \\
\hline 38 & 1 & 0 & -3.977424 & -0.141335 & -2.639860 \\
\hline 39 & 1 & 0 & -6.614583 & 1. 897292 & -0.109492 \\
\hline 40 & 1 & 0 & -7.164646 & 0.377010 & -0.877232 \\
\hline 41 & 1 & 0 & -7.033563 & 1. 867216 & -1.841960 \\
\hline 42 & 1 & 0 & -5.572183 & -5.737236 & 0.312781 \\
\hline 43 & 1 & 0 & -3.925071 & -5.798865 & -0.333860 \\
\hline 44 & 1 & 0 & -5.185301 & -4.918204 & -1.209049 \\
\hline 45 & 1 & 0 & -5.349347 & -3.269657 & 0.709003 \\
\hline 46 & 1 & 0 & -4.095744 & -4.143803 & 1. 571439 \\
\hline 47 & 1 & 0 & -2.476782 & -3.647039 & -0.280963 \\
\hline 48 & 1 & 0 & -3.728506 & -2.755185 & -1.134696 \\
\hline 49 & 1 & 0 & -3.896975 & -1.182011 & 0.873786 \\
\hline 50 & 1 & 0 & -2.575910 & -2.051224 & 1. 628171 \\
\hline 51 & 1 & 0 & -2.785134 & 3.915298 & 0.083155 \\
\hline 52 & 1 & 0 & -2.036812 & 5. 156171 & -0.942303 \\
\hline 53 & 1 & 0 & -1.787203 & 5. 195996 & 0.816192 \\
\hline 54 & 1 & 0 & 1. 377032 & 3.831907 & -0.577697 \\
\hline 55 & 1 & 0 & 0.677977 & 5.280085 & 0.196107 \\
\hline 56 & 1 & 0 & 0.393113 & 4. 976479 & -1.535085 \\
\hline 57 & 6 & 0 & 5. 994198 & -1.417469 & -1.747832 \\
\hline 58 & 6 & 0 & 7. 117410 & -0.630803 & -1.994350 \\
\hline 59 & 6 & 0 & 6.968182 & 0.717386 & -2.323753 \\
\hline 60 & 6 & 0 & 5.685312 & 1. 269072 & -2.404225 \\
\hline 61 & 6 & 0 & 4. 562704 & 0.483311 & -2.163083 \\
\hline 62 & 1 & 0 & 6. 116975 & -2.466354 & -1.488079 \\
\hline 63 & 1 & 0 & 8. 108857 & -1.070005 & -1.925478 \\
\hline 64 & 1 & 0 & 7.841504 & 1. 334937 & -2.513920 \\
\hline 65 & 1 & 0 & 5. 560972 & 2. 318849 & -2.656320 \\
\hline 66 & 1 & 0 & 3.576549 & 0.934088 & -2.226177 \\
\hline 67 & 6 & 0 & 1. 140092 & -2.516488 & -1.700288 \\
\hline 68 & 1 & 0 & 1. 293225 & -3.247980 & -0.897203 \\
\hline 69 & 1 & 0 & 1. 238796 & -3.071299 & -2.645794 \\
\hline
\end{tabular}




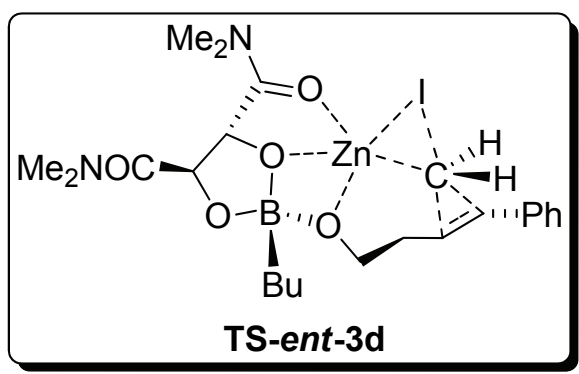

Imaginary frequency: $-367.2 \mathrm{~cm}^{-1}$

Standard orientation:

\begin{tabular}{|c|c|c|c|c|c|}
\hline \multirow{2}{*}{$\begin{array}{l}\text { Center } \\
\text { Number }\end{array}$} & \multirow{2}{*}{$\begin{array}{l}\text { Atomic } \\
\text { Number }\end{array}$} & \multirow{2}{*}{$\begin{array}{l}\text { Atomic } \\
\text { Type }\end{array}$} & \multicolumn{2}{|c|}{ Coordinates } & (Angstroms) \\
\hline & & & X & Y & Z \\
\hline 1 & 6 & 0 & 2. 395945 & -0.430590 & 0.392740 \\
\hline 2 & 30 & 0 & 0.410247 & -0.594337 & 0.225166 \\
\hline 3 & 8 & 0 & -0.889699 & -1.927639 & 1. 064663 \\
\hline 4 & 6 & 0 & -1.921071 & -2.102687 & 0.360214 \\
\hline 5 & 7 & 0 & -2.540001 & -3.289552 & 0.344126 \\
\hline 6 & 6 & 0 & -1.952391 & -4.419260 & 1. 068787 \\
\hline 7 & 8 & 0 & -1.334697 & -0.118654 & -0.854384 \\
\hline 8 & 5 & 0 & -1.475648 & 1. 315811 & -0.312751 \\
\hline 9 & 6 & 0 & -1.150960 & 2. 450396 & -1.408976 \\
\hline 10 & 6 & 0 & -1.786348 & 3.831300 & -1.158203 \\
\hline 11 & 6 & 0 & -1.522737 & 4.847983 & -2.277730 \\
\hline 12 & 6 & 0 & -2.168081 & 6.215262 & -2.025415 \\
\hline 13 & 8 & 0 & -2.848229 & 1. 350663 & 0.169390 \\
\hline 14 & 6 & 0 & -3.292165 & 0.055320 & 0.476887 \\
\hline 15 & 6 & 0 & -4.809779 & -0.062872 & 0.226285 \\
\hline 16 & 7 & 0 & -5.626830 & 0.751689 & 0.952186 \\
\hline 17 & 6 & 0 & -7.068861 & 0.657297 & 0.764516 \\
\hline 18 & 6 & 0 & -2.446578 & -0.899631 & -0.440514 \\
\hline 19 & 8 & 0 & -0.464763 & 1. 204456 & 0.825697 \\
\hline 20 & 6 & 0 & 0.106645 & 2. 296912 & 1.509056 \\
\hline 21 & 6 & 0 & 2. 566091 & 1. 604635 & 1. 290919 \\
\hline 22 & 6 & 0 & 3.783225 & 1. 170512 & 1. 743355 \\
\hline 23 & 1 & 0 & 3. 816341 & 0.754100 & 2.751132 \\
\hline 24 & 53 & 0 & 1. 859437 & -2.694632 & -1.271317 \\
\hline 25 & 6 & 0 & 5. 067752 & 1. 197541 & 1. 047812 \\
\hline 26 & 8 & 0 & -5.255476 & -0.880371 & -0.586103 \\
\hline 27 & 6 & 0 & -5.187875 & 1.736260 & 1. 933280 \\
\hline 28 & 6 & 0 & -3.719634 & -3.618455 & -0.461285 \\
\hline 29 & 1 & 0 & -3.024963 & -1.199780 & -1.314164 \\
\hline 30 & 1 & 0 & -3.090475 & -0.190605 & 1. 534078 \\
\hline 31 & 1 & 0 & 0.326489 & 3. 116529 & 0.809888 \\
\hline
\end{tabular}




\begin{tabular}{|c|c|c|c|c|c|}
\hline 32 & 1 & 0 & -0.605474 & 2.684236 & 2. 252515 \\
\hline 33 & 1 & 0 & 3. 054584 & -0.149023 & -0.423701 \\
\hline 34 & 1 & 0 & 2. 924337 & -1.065756 & 1. 104350 \\
\hline 35 & 1 & 0 & 2.512451 & 2. 104337 & 0.325004 \\
\hline 36 & 1 & 0 & -5.484120 & 1.430245 & 2.946456 \\
\hline 37 & 1 & 0 & -5.665543 & 2. 699961 & 1. 716693 \\
\hline 38 & 1 & 0 & -4.111899 & 1. 882191 & 1.881351 \\
\hline 39 & 1 & 0 & -7.277096 & -0.124493 & 0.035961 \\
\hline 40 & 1 & 0 & -7.468858 & 1. 613160 & 0.402804 \\
\hline 41 & 1 & 0 & -7.559650 & 0.415662 & 1.716463 \\
\hline 42 & 1 & 0 & -1.963381 & 6.916445 & -2.843287 \\
\hline 43 & 1 & 0 & -1.791477 & 6.666769 & -1.098458 \\
\hline 44 & 1 & 0 & -3.257474 & 6.126350 & -1.927942 \\
\hline 45 & 1 & 0 & -1.892149 & 4. 437653 & -3.228446 \\
\hline 46 & 1 & 0 & -0.437433 & 4. 973904 & -2.406061 \\
\hline 47 & 1 & 0 & -1.426494 & 4. 255491 & -0.207804 \\
\hline 48 & 1 & 0 & -2.870152 & 3.704828 & -1.031610 \\
\hline 49 & 1 & 0 & -1.517347 & 2. 077684 & -2.377259 \\
\hline 50 & 1 & 0 & -0.062624 & 2. 569931 & -1.543071 \\
\hline 51 & 1 & 0 & -4.289510 & -2.727475 & -0.721007 \\
\hline 52 & 1 & 0 & -4.365806 & -4.272937 & 0.132760 \\
\hline 53 & 1 & 0 & -3.417736 & -4.160178 & -1.366171 \\
\hline 54 & 1 & 0 & -1.007627 & -4.113657 & 1.511634 \\
\hline 55 & 1 & 0 & -1.778438 & -5.243123 & 0.368271 \\
\hline 56 & 1 & 0 & -2.641768 & -4.758471 & 1.850646 \\
\hline 57 & 6 & 0 & 6. 182078 & 0.596131 & 1. 664963 \\
\hline 58 & 6 & 0 & 7. 432851 & 0.600074 & 1. 052637 \\
\hline 59 & 6 & 0 & 7. 598299 & 1. 206290 & -0.194137 \\
\hline 60 & 6 & 0 & 6.503744 & 1. 812813 & -0.819469 \\
\hline 61 & 6 & 0 & 5. 254984 & 1.813560 & -0.206943 \\
\hline 62 & 1 & 0 & 6. 056721 & 0.117895 & 2.633575 \\
\hline 63 & 1 & 0 & 8.277105 & 0.127157 & 1.546494 \\
\hline 64 & 1 & 0 & 8. 571926 & 1. 209296 & -0.675882 \\
\hline 65 & 1 & 0 & 6.626649 & 2. 289989 & -1.787710 \\
\hline 66 & 1 & 0 & 4. 421233 & 2. 300983 & -0.703731 \\
\hline 67 & 6 & 0 & 1. 396214 & 1. 877149 & 2.218601 \\
\hline 68 & 1 & 0 & 1. 682671 & 2.704924 & 2.884442 \\
\hline 69 & 1 & 0 & 1. 200282 & 1. 014051 & 2. 867783 \\
\hline
\end{tabular}




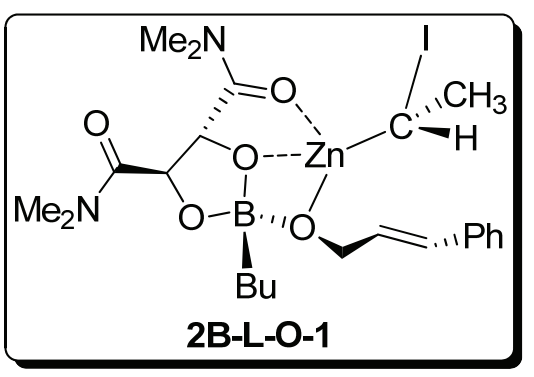

Standard orientation:

\begin{tabular}{|c|c|c|c|c|c|}
\hline \multirow{2}{*}{$\begin{array}{l}\text { Center } \\
\text { Number }\end{array}$} & \multirow{2}{*}{$\begin{array}{l}\text { Atomic } \\
\text { Number }\end{array}$} & \multirow{2}{*}{$\begin{array}{l}\text { Atomic } \\
\text { Type }\end{array}$} & \multicolumn{2}{|c|}{ Coordinates } & \multirow{2}{*}{$\begin{array}{r}(\text { Angstroms) } \\
Z\end{array}$} \\
\hline & & & $X$ & Y & \\
\hline 1 & 6 & 0 & 6.728226 & -2.625142 & -2.380469 \\
\hline 2 & 6 & 0 & 6.737993 & -2.659362 & -0.985588 \\
\hline 3 & 6 & 0 & 5.535862 & -2.664259 & -0.279172 \\
\hline 4 & 6 & 0 & 4. 298376 & -2.629995 & -0.946449 \\
\hline 5 & 6 & 0 & 4. 306950 & -2.603961 & -2.354039 \\
\hline 6 & 6 & 0 & 5.506201 & -2.599102 & -3.060340 \\
\hline 7 & 6 & 0 & 3. 063159 & -2.624453 & -0.147728 \\
\hline 8 & 6 & 0 & 1.804118 & -2.448956 & -0.580076 \\
\hline 9 & 6 & 0 & 0.606852 & -2.484002 & 0.321057 \\
\hline 10 & 8 & 0 & -0.100690 & -1.248368 & 0.232524 \\
\hline 11 & 30 & 0 & 0.553417 & 0.674043 & 0.299244 \\
\hline 12 & 8 & 0 & -0.333410 & 1.522245 & -1.353501 \\
\hline 13 & 6 & 0 & -1.562462 & 1. 767550 & -1.227631 \\
\hline 14 & 7 & 0 & -2.138627 & 2.738476 & -1.951344 \\
\hline 15 & 6 & 0 & -1.314195 & 3.563254 & -2.837413 \\
\hline 16 & 5 & 0 & -1.472434 & -1.024713 & 0.900787 \\
\hline 17 & 6 & 0 & -1.589576 & -1.578199 & 2. 406332 \\
\hline 18 & 6 & 0 & -2.038064 & -3.044055 & 2.558080 \\
\hline 19 & 6 & 0 & -2.235509 & -3.481823 & 4. 016341 \\
\hline 20 & 6 & 0 & -2.689936 & -4.938425 & 4. 160328 \\
\hline 21 & 8 & 0 & -2.558109 & -1.441380 & 0.029982 \\
\hline 22 & 6 & 0 & -2.837922 & -0.440816 & -0.915171 \\
\hline 23 & 6 & 0 & -4.343923 & -0.448533 & -1.252589 \\
\hline 24 & 7 & 0 & -4.846377 & -1.577727 & -1.826206 \\
\hline 25 & 6 & 0 & -6.260450 & -1.625279 & -2.176419 \\
\hline 26 & 6 & 0 & -2.369360 & 0.901413 & -0.245950 \\
\hline 27 & 8 & 0 & -1.505124 & 0.504293 & 0.807135 \\
\hline 28 & 6 & 0 & 2. 178180 & 1.528470 & 1. 057057 \\
\hline 29 & 6 & 0 & 3.441806 & 1.554789 & 0.210712 \\
\hline 30 & 53 & 0 & 1.564530 & 3.680661 & 1.516626 \\
\hline 31 & 8 & 0 & -5.050500 & 0.538158 & -1.019906 \\
\hline 32 & 6 & 0 & -4.085522 & -2.782704 & -2.133331 \\
\hline
\end{tabular}




\begin{tabular}{|c|c|c|c|c|c|}
\hline 33 & 6 & 0 & -3.539662 & 3. 155686 & -1.851788 \\
\hline 34 & 1 & 0 & -3.225900 & 1. 434111 & 0.168396 \\
\hline 35 & 1 & 0 & -2.264592 & -0.602914 & -1.844459 \\
\hline 36 & 1 & 0 & 0.910582 & -2.682157 & 1. 358197 \\
\hline 37 & 1 & 0 & -0.075831 & -3.289534 & 0.014208 \\
\hline 38 & 1 & 0 & 2. 402818 & 1. 183617 & 2. 068247 \\
\hline 39 & 1 & 0 & 1. 591281 & -2.269252 & -1.633379 \\
\hline 40 & 1 & 0 & -3.984690 & -2.910558 & -3.220214 \\
\hline 41 & 1 & 0 & -4.618147 & -3.656048 & -1.737260 \\
\hline 42 & 1 & 0 & -3.105628 & -2.753637 & -1.663644 \\
\hline 43 & 1 & 0 & -6.718209 & -0.672338 & -1.916421 \\
\hline 44 & 1 & 0 & -6.759917 & -2.434614 & -1.628927 \\
\hline 45 & 1 & 0 & -6.378664 & -1.811285 & -3.251984 \\
\hline 46 & 1 & 0 & -2.822074 & -5.218267 & 5.212313 \\
\hline 47 & 1 & 0 & -1.956561 & -5.628094 & 3. 722933 \\
\hline 48 & 1 & 0 & -3.645821 & -5.107771 & 3.648589 \\
\hline 49 & 1 & 0 & -2.971679 & -2.818708 & 4. 492958 \\
\hline 50 & 1 & 0 & -1.295802 & -3.335135 & 4. 568827 \\
\hline 51 & 1 & 0 & -1.310014 & -3.721575 & 2. 086569 \\
\hline 52 & 1 & 0 & -2.977783 & -3.187947 & 2. 007073 \\
\hline 53 & 1 & 0 & -2.323023 & -0.942448 & 2.925026 \\
\hline 54 & 1 & 0 & -0.643475 & -1.428764 & 2. 952233 \\
\hline 55 & 1 & 0 & -4.163177 & 2. 377727 & -1.413552 \\
\hline 56 & 1 & 0 & -3.910157 & 3.357626 & -2.862411 \\
\hline 57 & 1 & 0 & -3.614400 & 4. 080994 & -1.266881 \\
\hline 58 & 1 & 0 & -0.274521 & 3.255025 & -2.757214 \\
\hline 59 & 1 & 0 & -1.409764 & 4. 615099 & -2.545450 \\
\hline 60 & 1 & 0 & -1.657989 & 3. 452963 & -3.872285 \\
\hline 61 & 1 & 0 & 5. 549822 & -2.690513 & 0.808088 \\
\hline 62 & 1 & 0 & 3.368055 & -2.596547 & -2.900099 \\
\hline 63 & 1 & 0 & 7. 680934 & -2.683144 & -0.445966 \\
\hline 64 & 1 & 0 & 5. 489510 & -2.579829 & -4.146895 \\
\hline 65 & 1 & 0 & 7. 662465 & -2.623319 & -2.935199 \\
\hline 66 & 1 & 0 & 3.213049 & -2.778253 & 0.922081 \\
\hline 67 & 1 & 0 & 4. 268646 & 2. 096337 & 0.687601 \\
\hline 68 & 1 & 0 & 3. 770113 & 0.517229 & 0.049083 \\
\hline 69 & 1 & 0 & 3. 270943 & 2. 001758 & -0.774158 \\
\hline
\end{tabular}




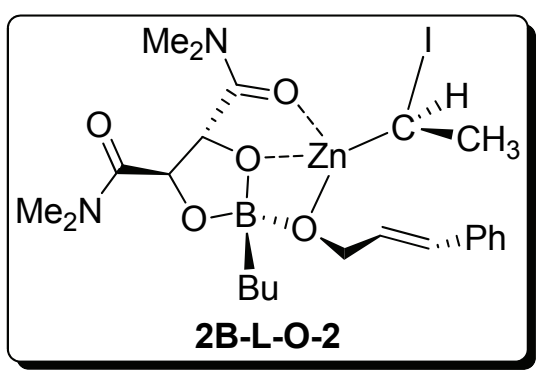

Standard orientation:

\begin{tabular}{|c|c|c|c|c|c|}
\hline \multirow{2}{*}{$\begin{array}{l}\text { Center } \\
\text { Number }\end{array}$} & \multirow{2}{*}{$\begin{array}{l}\text { Atomic } \\
\text { Number }\end{array}$} & \multirow{2}{*}{$\begin{array}{l}\text { Atomic } \\
\text { Type }\end{array}$} & \multicolumn{2}{|c|}{ Coordinates } & \multirow{2}{*}{$\begin{array}{r}(\text { Angstroms) } \\
Z\end{array}$} \\
\hline & & & X & Y & \\
\hline 1 & 6 & 0 & -2.368990 & 1.001479 & -0.145444 \\
\hline 2 & 6 & 0 & -2.906029 & -0.281343 & -0.877659 \\
\hline 3 & 8 & 0 & -2.640117 & -1.344522 & 0.000225 \\
\hline 4 & 5 & 0 & -1.511999 & -1.021767 & 0.857528 \\
\hline 5 & 8 & 0 & -1.490243 & 0.512017 & 0.854828 \\
\hline 6 & 8 & 0 & -0.172598 & -1.255186 & 0.133935 \\
\hline 7 & 30 & 0 & 0.558092 & 0.639105 & 0.288737 \\
\hline 8 & 6 & 0 & 0.503927 & -2.511470 & 0.146294 \\
\hline 9 & 6 & 0 & 2.245090 & 1. 314591 & 1. 090228 \\
\hline 10 & 53 & 0 & 1.973173 & 3.566849 & 1. 337524 \\
\hline 11 & 6 & 0 & 1.689319 & -2.455697 & -0.769501 \\
\hline 12 & 6 & 0 & 2.951467 & -2.667201 & -0.362991 \\
\hline 13 & 6 & 0 & -4.419109 & -0.215627 & -1.173427 \\
\hline 14 & 8 & 0 & -5.082848 & 0.779920 & -0.864746 \\
\hline 15 & 7 & 0 & -4.976824 & -1.290415 & -1.798132 \\
\hline 16 & 6 & 0 & -4.270901 & -2.504084 & -2.190507 \\
\hline 17 & 6 & 0 & -6.400257 & -1.268075 & -2.110788 \\
\hline 18 & 6 & 0 & -2.787917 & -5.070348 & 3.923345 \\
\hline 19 & 6 & 0 & -2.283132 & -3.624964 & 3.849576 \\
\hline 20 & 6 & 0 & -2.106420 & -3.111720 & 2.413533 \\
\hline 21 & 6 & 0 & -1.608456 & -1.656036 & 2. 332381 \\
\hline 22 & 6 & 0 & -1.558505 & 1. 893718 & -1.100311 \\
\hline 23 & 8 & 0 & -0.342881 & 1.612538 & -1.276033 \\
\hline 24 & 7 & 0 & -2.116382 & 2.924141 & -1.751646 \\
\hline 25 & 6 & 0 & -3.498951 & 3.382478 & -1.593019 \\
\hline 26 & 6 & 0 & -1.286577 & 3.765317 & -2.617559 \\
\hline 27 & 1 & 0 & -3.193337 & 1.539076 & 0.324519 \\
\hline 28 & 1 & 0 & -2.363337 & -0.408993 & -1.830479 \\
\hline 29 & 1 & 0 & 0.817136 & -2.771021 & 1. 166794 \\
\hline 30 & 1 & 0 & -0.202937 & -3.282791 & -0.192028 \\
\hline 31 & 1 & 0 & 3. 077112 & 1. 306019 & 0.383371 \\
\hline 3 & 1 & 0 & 1. 464973 & -2.226055 & -1.810603 \\
\hline
\end{tabular}




\begin{tabular}{|c|c|c|c|c|c|}
\hline 33 & 1 & 0 & -4.214825 & -2.581216 & -3.285268 \\
\hline 34 & 1 & 0 & -4.819055 & -3.377592 & -1.816352 \\
\hline 35 & 1 & 0 & -3.273785 & -2.531530 & -1.758687 \\
\hline 36 & 1 & 0 & -6.813003 & -0.310397 & -1.798129 \\
\hline 37 & 1 & 0 & -6.917953 & -2.080387 & -1.584913 \\
\hline 38 & 1 & 0 & -6.552473 & -1.402639 & -3.189612 \\
\hline 39 & 1 & 0 & -2.904135 & -5.405411 & 4. 960961 \\
\hline 40 & 1 & 0 & -2.092038 & -5.759484 & 3. 427741 \\
\hline 41 & 1 & 0 & -3.761914 & -5.175258 & 3. 428848 \\
\hline 42 & 1 & 0 & -2.981836 & -2.964417 & 4. 382827 \\
\hline 43 & 1 & 0 & -1.324880 & -3.543769 & 4. 383284 \\
\hline 44 & 1 & 0 & -1.415800 & -3.786348 & 1.885038 \\
\hline 45 & 1 & 0 & -3.064122 & -3.190347 & 1. 880879 \\
\hline 46 & 1 & 0 & -2.304269 & -1.026137 & 2. 907036 \\
\hline 47 & 1 & 0 & -0.643377 & -1.571547 & 2. 858590 \\
\hline 48 & 1 & 0 & -4.137663 & 2.605590 & -1.175335 \\
\hline 49 & 1 & 0 & -3.889087 & 3.646198 & -2.581833 \\
\hline 50 & 1 & 0 & -3.525434 & 4. 280155 & -0.962664 \\
\hline 51 & 1 & 0 & -0.252890 & 3. 431622 & -2.567235 \\
\hline 52 & 1 & 0 & -1.354400 & 4. 805935 & -2.281581 \\
\hline 53 & 1 & 0 & -1.648618 & 3.705112 & -3.650410 \\
\hline 54 & 6 & 0 & 4. 176492 & -2.651482 & -1.176506 \\
\hline 55 & 6 & 0 & 5.419483 & -2.770078 & -0.530038 \\
\hline 56 & 6 & 0 & 4. 170341 & -2.521882 & -2.578295 \\
\hline 57 & 6 & 0 & 6.613367 & -2.749886 & -1.249963 \\
\hline 58 & 1 & 0 & 5.444718 & -2.874341 & 0.552342 \\
\hline 59 & 6 & 0 & 5.361263 & -2.501316 & -3.298288 \\
\hline 60 & 1 & 0 & 3.226458 & -2.443156 & -3.109911 \\
\hline 61 & 6 & 0 & 6.589342 & -2.613879 & -2.638488 \\
\hline 62 & 1 & 0 & 7. 561049 & -2.840465 & -0.725930 \\
\hline 63 & 1 & 0 & 5.333170 & -2.400696 & -4.380119 \\
\hline 64 & 1 & 0 & 7. 517059 & -2.598790 & -3.203874 \\
\hline 65 & 1 & 0 & 3.112581 & -2.875404 & 0.696077 \\
\hline 66 & 6 & 0 & 2. 680927 & 0.728926 & 2. 424719 \\
\hline 67 & 1 & 0 & 2. 882607 & -0.343008 & 2. 276015 \\
\hline 68 & 1 & 0 & 3.596622 & 1. 186873 & 2. 819878 \\
\hline 69 & 1 & 0 & 1.904043 & 0.814567 & 3. 191242 \\
\hline
\end{tabular}




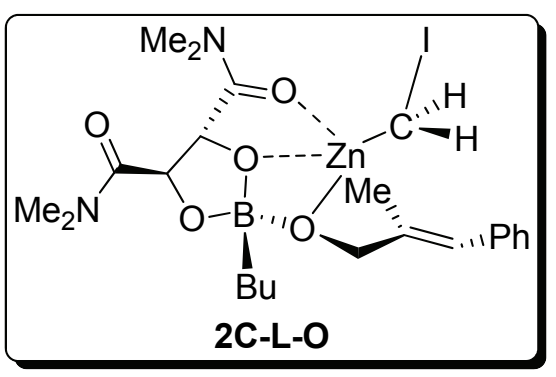

Standard orientation:

\begin{tabular}{|c|c|c|c|c|c|}
\hline & Atomic & Atomic & \multicolumn{2}{|c|}{ Coordinates } & (Angstroms) \\
\hline Number & Number & Tyре & $X$ & Y & Z \\
\hline 1 & 6 & 0 & -2.348332 & 1. 071399 & -0.155520 \\
\hline 2 & 6 & 0 & -2.908644 & -0.235799 & -0.828697 \\
\hline 3 & 8 & 0 & -2.621882 & -1.273983 & 0.071131 \\
\hline 4 & 5 & 0 & -1.519187 & -0.911032 & 0.943851 \\
\hline 5 & 8 & 0 & -1.517281 & 0.620846 & 0.901019 \\
\hline 6 & 8 & 0 & -0.158823 & -1.146535 & 0.257887 \\
\hline 7 & 30 & 0 & 0.559645 & 0.743187 & 0.447395 \\
\hline 8 & 6 & 0 & 0.508789 & -2.408075 & 0.268415 \\
\hline 9 & 6 & 0 & 2.174707 & 1. 482010 & 1. 309937 \\
\hline 10 & 53 & 0 & 2. 068820 & 3. 717482 & 1. 388820 \\
\hline 11 & 6 & 0 & 1. 695596 & -2.377938 & -0.667281 \\
\hline 12 & 6 & 0 & 2.931947 & -2.544337 & -0.155281 \\
\hline 13 & 6 & 0 & -4.432611 & -0.171816 & -1.076924 \\
\hline 14 & 8 & 0 & -5.080459 & 0.838884 & -0.784085 \\
\hline 15 & 7 & 0 & -5.015447 & -1.265262 & -1.642977 \\
\hline 16 & 6 & 0 & -4.330362 & -2.498125 & -2.012235 \\
\hline 17 & 6 & 0 & -6.449292 & -1.247087 & -1.905380 \\
\hline 18 & 6 & 0 & -2.794540 & -4.890216 & 4. 100431 \\
\hline 19 & 6 & 0 & -2.311204 & -3.439682 & 3.993603 \\
\hline 20 & 6 & 0 & -2.112020 & -2.966251 & 2.546779 \\
\hline 21 & 6 & 0 & -1.636765 & -1.505302 & 2. 433666 \\
\hline 22 & 6 & 0 & -1.490768 & 1.885887 & -1.139656 \\
\hline 23 & 8 & 0 & -0.261180 & 1. 623844 & -1.213472 \\
\hline 24 & 7 & 0 & -2.027858 & 2.832004 & -1.924623 \\
\hline 25 & 6 & 0 & -3.422778 & 3.276796 & -1.890186 \\
\hline 26 & 6 & 0 & -1.164398 & 3.596281 & -2.827946 \\
\hline 27 & 1 & 0 & -3.168917 & 1. 661275 & 0.255451 \\
\hline 28 & 1 & 0 & -2.402023 & -0.398168 & -1.796198 \\
\hline 29 & 1 & 0 & 0.826429 & -2.662597 & 1. 287545 \\
\hline 30 & 1 & 0 & -0.210023 & -3.172858 & -0.060760 \\
\hline 31 & 1 & 0 & 2.308825 & 1. 207623 & 2. 356810 \\
\hline 32 & 1 & 0 & 3. 110241 & 1. 297854 & 0.780554 \\
\hline
\end{tabular}




\begin{tabular}{|c|c|c|c|c|c|}
\hline 33 & 1 & 0 & -4.318197 & -2.619632 & -3.104299 \\
\hline 34 & 1 & 0 & -4.866914 & -3.352706 & -1.581775 \\
\hline 35 & 1 & 0 & -3.317068 & -2.513987 & -1.619441 \\
\hline 36 & 1 & 0 & -6.845332 & -0.273012 & -1.623347 \\
\hline 37 & 1 & 0 & -6.952420 & -2.030267 & -1.324068 \\
\hline 38 & 1 & 0 & -6.640365 & -1.430693 & -2.970633 \\
\hline 39 & 1 & 0 & -2.927349 & -5.195817 & 5. 145126 \\
\hline 40 & 1 & 0 & -2.077451 & -5.582358 & 3.640606 \\
\hline 41 & 1 & 0 & -3.756155 & -5.026063 & 3.589429 \\
\hline 42 & 1 & 0 & -3.031386 & -2.775057 & 4. 492107 \\
\hline 43 & 1 & 0 & -1.365909 & -3.327031 & 4. 544399 \\
\hline 44 & 1 & 0 & -1.398656 & -3.643609 & 2.053370 \\
\hline 45 & 1 & 0 & -3.056866 & -3.077101 & 1. 996925 \\
\hline 46 & 1 & 0 & -2.354632 & -0.871328 & 2. 975927 \\
\hline 47 & 1 & 0 & -0.683935 & -1.390376 & 2.976136 \\
\hline 48 & 1 & 0 & -4.067459 & 2.563323 & -1.378686 \\
\hline 49 & 1 & 0 & -3.779265 & 3.381221 & -2.920843 \\
\hline 50 & 1 & 0 & -3.487520 & 4. 258231 & -1.403906 \\
\hline 51 & 1 & 0 & -0.132649 & 3.277210 & -2.700824 \\
\hline 52 & 1 & 0 & -1.251375 & 4. 663596 & -2.595419 \\
\hline 53 & 1 & 0 & -1.480101 & 3. 436442 & -3.865360 \\
\hline 54 & 6 & 0 & 4. 234206 & -2.593271 & -0.845443 \\
\hline 55 & 6 & 0 & 5.364204 & -2.069596 & -0.188647 \\
\hline 56 & 6 & 0 & 4. 420454 & -3.176341 & -2.112320 \\
\hline 57 & 6 & 0 & 6.621625 & -2.089788 & -0.787589 \\
\hline 58 & 1 & 0 & 5.244768 & -1.632577 & 0.800109 \\
\hline 59 & 6 & 0 & 5.680688 & -3.203045 & -2.710049 \\
\hline 60 & 1 & 0 & 3.580325 & -3.640571 & -2.618272 \\
\hline 61 & 6 & 0 & 6.784755 & -2.653906 & -2.055377 \\
\hline 62 & 1 & 0 & 7. 475709 & -1.669234 & -0.263344 \\
\hline 63 & 1 & 0 & 5.801241 & -3.664491 & -3.686810 \\
\hline 64 & 1 & 0 & 7. 765428 & -2.675996 & -2.522837 \\
\hline 65 & 1 & 0 & 3.003275 & -2.632309 & 0.929775 \\
\hline 66 & 6 & 0 & 1. 361631 & -2.130218 & -2.117863 \\
\hline 67 & 1 & 0 & 2.225225 & -1.785910 & -2.691515 \\
\hline 68 & 1 & 0 & 0.974578 & -3.039160 & -2.601287 \\
\hline 69 & 1 & 0 & 0.573856 & -1.371978 & -2.188487 \\
\hline
\end{tabular}




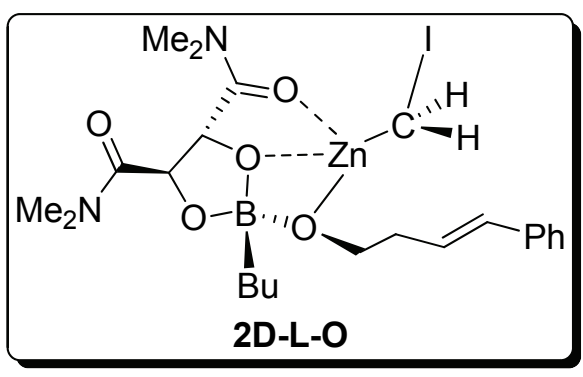

Standard orientation:

\begin{tabular}{|c|c|c|c|c|c|}
\hline \multirow{2}{*}{$\begin{array}{l}\text { Center } \\
\text { Number }\end{array}$} & \multirow{2}{*}{$\begin{array}{l}\text { Atomic } \\
\text { Number }\end{array}$} & \multirow{2}{*}{$\begin{array}{l}\text { Atomic } \\
\text { Type }\end{array}$} & \multicolumn{2}{|c|}{ Coordinates } & (Angstroms) \\
\hline & & & $X$ & Y & Z \\
\hline 1 & 6 & 0 & 2.065809 & -1.899125 & 0.903485 \\
\hline 2 & 30 & 0 & 0.722438 & -0.769307 & -0.015678 \\
\hline 3 & 8 & 0 & 0.960269 & 1. 267302 & -0.213444 \\
\hline 4 & 6 & 0 & 0.103606 & 1.994830 & 0.348655 \\
\hline 5 & 7 & 0 & 0.351734 & 3.298675 & 0.561672 \\
\hline 6 & 6 & 0 & 1. 643231 & 3.867675 & 0.176056 \\
\hline 7 & 8 & 0 & -1.136074 & -0.025790 & 0.772969 \\
\hline 8 & 5 & 0 & -1.960760 & -0.620077 & -0.361902 \\
\hline 9 & 6 & 0 & -3.013751 & -1.705876 & 0.184024 \\
\hline 10 & 6 & 0 & -3.806613 & -2.487044 & -0.878135 \\
\hline 11 & 6 & 0 & -4.864073 & -3.436053 & -0.294858 \\
\hline 12 & 6 & 0 & -5.641618 & -4.215297 & -1.361347 \\
\hline 13 & 8 & 0 & -2.521972 & 0.563760 & -1.028920 \\
\hline 14 & 6 & 0 & -2.386171 & 1. 709491 & -0.244328 \\
\hline 15 & 6 & 0 & -3.649791 & 2. 188772 & 0.529119 \\
\hline 16 & 7 & 0 & -4.893678 & 2.000078 & 0.008850 \\
\hline 17 & 6 & 0 & -6.041677 & 2.466596 & 0.780153 \\
\hline 18 & 6 & 0 & -1.237656 & 1.379072 & 0.779435 \\
\hline 19 & 8 & 0 & -0.867378 & -1.243905 & -1.232743 \\
\hline 20 & 6 & 0 & -0.971776 & -1.244822 & -2.650648 \\
\hline 21 & 6 & 0 & 1. 470463 & -0.672572 & -2.989897 \\
\hline 22 & 6 & 0 & 2.683573 & -1.016725 & -2.521797 \\
\hline 23 & 1 & 0 & 2.868231 & -2.072963 & -2.321602 \\
\hline 24 & 6 & 0 & 3.831250 & -0.133645 & -2.257607 \\
\hline 25 & 8 & 0 & -3.477281 & 2. 787594 & 1. 598713 \\
\hline 26 & 6 & 0 & -5.218271 & 1. 530538 & -1.334567 \\
\hline 27 & 6 & 0 & -0.549621 & 4. 224869 & 1. 248401 \\
\hline 28 & 1 & 0 & -1.510274 & 1.721442 & 1. 778623 \\
\hline 29 & 1 & 0 & -2.099542 & 2.558826 & -0.885912 \\
\hline 30 & 1 & 0 & -1.750707 & -1.954741 & -2.955699 \\
\hline 31 & 1 & 0 & -1.274898 & -0.248146 & -2.996681 \\
\hline 32 & 1 & 0 & 1.859678 & -2.970390 & 0.918287 \\
\hline
\end{tabular}




\begin{tabular}{|c|c|c|c|c|c|}
\hline 33 & 1 & 0 & 3. 096194 & -1.743934 & 0.582486 \\
\hline 34 & 1 & 0 & 1. 245900 & 0.374065 & -3.197062 \\
\hline 35 & 1 & 0 & -5.724924 & 2.331582 & -1.891023 \\
\hline 36 & 1 & 0 & -5.902296 & 0.674908 & -1.272362 \\
\hline 37 & 1 & 0 & -4.323293 & 1. 215623 & -1.861267 \\
\hline 38 & 1 & 0 & -5.717827 & 2. 708919 & 1. 790601 \\
\hline 39 & 1 & 0 & -6.804847 & 1. 680165 & 0.808358 \\
\hline 40 & 1 & 0 & -6.482786 & 3. 360889 & 0.318674 \\
\hline 41 & 1 & 0 & -6.385750 & -4.883840 & -0.912212 \\
\hline 42 & 1 & 0 & -4.968283 & -4.830072 & -1.972197 \\
\hline 43 & 1 & 0 & -6.172162 & -3.535846 & -2.041015 \\
\hline 44 & 1 & 0 & -5.566556 & -2.856367 & 0.321474 \\
\hline 45 & 1 & 0 & -4.373581 & -4.141523 & 0.390870 \\
\hline 46 & 1 & 0 & -3.112924 & -3.077048 & -1.495781 \\
\hline 47 & 1 & 0 & -4.303548 & -1.785878 & -1.566929 \\
\hline 48 & 1 & 0 & -3.726278 & -1.190297 & 0.848640 \\
\hline 49 & 1 & 0 & -2.484276 & -2.424775 & 0.828828 \\
\hline 50 & 1 & 0 & -1.528950 & 3.786422 & 1. 441254 \\
\hline 51 & 1 & 0 & -0.682086 & 5. 119363 & 0.628168 \\
\hline 52 & 1 & 0 & -0.103019 & 4. 533499 & 2. 201889 \\
\hline 53 & 1 & 0 & 2. 260421 & 3. 095997 & -0.279544 \\
\hline 54 & 1 & 0 & 2. 148982 & 4. 264601 & 1. 064107 \\
\hline 55 & 1 & 0 & 1. 488661 & 4. 689384 & -0.533333 \\
\hline 56 & 6 & 0 & 4. 991029 & -0.685599 & -1.685953 \\
\hline 57 & 6 & 0 & 6.108429 & 0.102656 & -1.411054 \\
\hline 58 & 6 & 0 & 6.092182 & 1. 465257 & -1.708816 \\
\hline 59 & 6 & 0 & 4. 949228 & 2. 029597 & -2.285352 \\
\hline 60 & 6 & 0 & 3.833859 & 1. 242098 & -2.557219 \\
\hline 61 & 1 & 0 & 5.012054 & -1.747944 & -1.453761 \\
\hline 62 & 1 & 0 & 6.990182 & -0.349242 & -0.965020 \\
\hline 63 & 1 & 0 & 6.961089 & 2. 082907 & -1.499067 \\
\hline 64 & 1 & 0 & 4. 931907 & 3.088446 & -2.531132 \\
\hline 65 & 1 & 0 & 2. 960190 & 1. 697735 & -3.013489 \\
\hline 66 & 6 & 0 & 0.361548 & -1.643673 & -3.287726 \\
\hline 67 & 1 & 0 & 0.640434 & -2.653689 & -2.962251 \\
\hline 68 & 1 & 0 & 0.197838 & -1.693054 & -4.375839 \\
\hline 69 & 53 & 0 & 2. 152484 & -1.375369 & 3. 081026 \\
\hline
\end{tabular}




\section{Computed Energies of All Stationary Points}

Table S1. Sum of electronic and thermal enthalpies ( $H$, in Hartree), sum of electronic and thermal free energies ( $G$, in Hartree), thermal correction to Enthalpy ( $T C H$, in Hartree), thermal correction to Gibbs free energy (TCGFE, in Hartree), electronic energy $\left(E\right.$, in Hartree), and total free energy in solution $\left(E_{\mathrm{S}}\right.$, in Hartree, solvent $=$ dichloromethane)

\begin{tabular}{|c|c|c|c|c|c|c|}
\hline Structure & $H^{\mathrm{a}}$ & $G^{\mathrm{a}}$ & $T C H^{\mathrm{a}}$ & $T C G F E^{\mathrm{a}}$ & $E^{\mathrm{b}}$ & $E_{\mathrm{S}}{ }^{\mathrm{b}}$ \\
\hline 2-M & -2253.263175 & -2253.32773 & 0.199005 & 0.13445 & -701.583042 & -701.591911 \\
\hline TS- & 1766 & -2253.277267 & 95937 & 3633 & -701.531696 & -701.553412 \\
\hline 2-D & -4506.596257 & -4506.703155 & 0.400527 & 0.293629 & -1403.227023 & -1403.227045 \\
\hline TS-2-D & -4506.563433 & -4506.669399 & 0.398312 & 0.292346 & -1403.188602 & -1403.193661 \\
\hline $2-T$ & 64383 & -9013.443432 & 0.803356 & 0.624307 & -2806.503472 & -2806.478574 \\
\hline TS-2-T & -9013.237511 & -9013.414269 & 0.801576 & 0.624818 & -2806.472745 & -2806.452713 \\
\hline $3-T$ & -9013.317553 & -9013.498515 & 80537 & 0.624407 & -2806.558674 & -2806.535439 \\
\hline 1 & -906.091234 & -906.170714 & 0.384388 & 0.304908 & -906.737136 & -906.731456 \\
\hline 2-L-O & -3159.425046 & -3159.541886 & 0.585558 & 0.468718 & -1608.378685 & -1608.365094 \\
\hline 3-L-O & -3159.4 & -3159.5 & 22 & 01 & -1608.432038 & -1608 . \\
\hline 2 & $-315 \mathrm{C}$ & -315 & 83 & 08 & -1608 & -16 \\
\hline $1^{\prime}$ & -873.587411 & -873.659371 & 0.375895 & 0.303935 & -874.199883 & -874.199454 \\
\hline 2-L' & -3126.875062 & -3126.988174 & 0.57664 & 0.463528 & -1575.797892 & -1575.792982 \\
\hline TS-3a & -3159.393242 & -3159.504344 & 0.583822 & 0.472719 & -1608.341597 & -1608.33995 \\
\hline TS-ent-3a & -3159.385174 & -3159.496527 & 0.583684 & 0.472331 & -1608.334588 & -1608.334415 \\
\hline 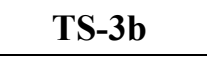 & -3198.676199 & 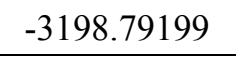 & 182 & 0.497391 & -1647.666163 & -1647.658915 \\
\hline TS-ent-3b & -3198.669387 & -3198.785874 & 0.612928 & 0.496441 & -1647.658937 & -1647.65138 \\
\hline TS-3-epi-3b & -3198.672186 & -3198.787197 & 0.613304 & 0.498294 & -1647.661217 & -1647.654863 \\
\hline TS-3c & -3198.675176 & -3198.788678 & 0.613549 & 0.500048 & -1647.663976 & -1647.657698 \\
\hline TS-ent-3c & -3198.668714 & -3198.783428 & 0.613096 & 0.498382 & -1647.659289 & -1647.653945 \\
\hline TS-3d & -3198.67748 & -3198.792091 & 0.613697 & 0.499086 & -1647.667962 & -1647.661263 \\
\hline TS-ent-3d & -3198.674937 & -3198.789983 & 0.614051 & 0.499005 & -1647.665188 & -1647.65933 \\
\hline 2B-L-O-1 & -3198.707192 & -3198.827211 & 0.615378 & 0.495359 & -1647.701469 & -1647.685149 \\
\hline 2B-L-O-2 & -3198.707111 & -3198.826942 & 0.615392 & 0.495561 & -1647.701454 & -1647.685375 \\
\hline 2C-L-O & -3198.70959 & -3198.829308 & 0.615402 & 0.495683 & -1647.703189 & -1647.684972 \\
\hline 2D-L-O & -3198.710307 & -3198.828269 & 0.615428 & 0.497465 & -1647.702509 & -1647.689905 \\
\hline
\end{tabular}

a Computed at the B3LYP level of theory with the Ahlrichs' SVP all-electron basis set for the zinc atom and the 6-31G(d) basis set for other atoms except for the iodine atom, for which the LANL2DZ basis set was used.

${ }^{\mathbf{b}}$ Computed at the B3LYP level of theory with the SDD basis set for zinc and iodine atoms and the $6-311 \mathrm{G}(\mathrm{d}, \mathrm{p})$ basis set for the other atoms. 


\section{Full Citation of Reference 11}

Frisch, M. J.; Trucks, G. W.; Schlegel, H. B.; Scuseria, G. E.; Robb, M. A.; Cheeseman, J. R.; Montgomery, J. A. Jr.; Vreven, T.; Kudin, K. N.; Burant, J. C.; Millam, J. M.; Iyengar, S. S.; Tomasi, J.; Barone, V.; Mennucci, B.; Cossi, M.; Scalmani, G.; Rega, N.; Petersson, G. A.; Nakatsuji, H.; Hada, M.; Ehara, M.; Toyota, K.; Fukuda, R.; Hasegawa, J.; Ishida, M.; Nakajima, T.; Honda, Y.; Kitao, O.; Nakai, H.; Klene, M.; Li, X.; Knox, J. E.; Hratchian, H. P.; Cross, J. B.; Adamo, C.; Jaramillo, J.; Gomperts, R.; Stratmann, R. E.; Yazyev, O.; Austin, A. J.; Cammi, R.; Pomelli, C.; Ochterski, J. W.; Ayala, P. Y.; Morokuma, K.; Voth, G. A.; Salvador, P.; Dannenberg, J. J.; Zakrzewski, V. G.; Dapprich, S.; Daniels, A. D.; Strain, M. C.; Farkas, O.; Malick, D. K.; Rabuck, A. D.; Raghavachari, K.; Foresman, J. B.; Ortiz, J. V.; Cui, Q.; Baboul, A. G.; Clifford, S.; Cioslowski, J.; Stefanov, B. B.; Liu, G.; Liashenko, A.; Piskorz, P.; Komaromi, I.; Martin, R. L.; Fox, D. J.; Keith, T.; Al-Laham, M. A.; Peng, C. Y.; Nanayakkara, A.; Challacombe, M.; Gill, P. M. W.;

Johnson, B.; Chen, W.; Wong, M. W.; Gonzalez, C.; Pople, J. A. Gaussian 03, Revision C.02; Gaussian Inc.: Wallingford CT, 2004. 


\section{Comparison of Different Basis Sets and Atomic Radii Sets}

In the initial submission of this paper, we conducted DFT calculations at the B3LYP level of theory using basis set B1 (the Ahlrichs' SVP all-electron basis set for the zinc atom and the 6-31G(d) basis set for other atoms except for the iodine atom, for which the LANL2DZ basis set was used). A reviewer suggested that we should check the basis set dependence and compare different basis sets. During the revision, six different basis sets B1-6 were compared. First, we conducted the comparison between experimental selectivities (enantioselectivity and diastereoselectivity) of reactions A-D (for details, see Scheme 2 in the paper) and DFT-calculation predicted ones using different basis sets B1-6 (Table S2). It was found that all these calculations can repeat the experimental results in some extent. This implies that the choice of different basis sets has limited influence on understanding and predicting the selectivities (enantioselectivity and diastereoselectivity) of reactions A-D. Among them, the calculations using basis set B5 (the SDD basis set for zinc and iodine atoms and the $6-311 \mathrm{G}(\mathrm{d}, \mathrm{p})$ basis set for the other atoms) give the best results as compared with the experimental data. In addition, according to this reviewer's suggestion, the solvent effects were also computed with UAKS radii using different basis sets B1-6 (Table S3). It was found that the results are very similar to those with UA0 radii. Therefore, we chose the basis set $\mathbf{B 5}$ and the UA0 radii for the DFT calculations in the revised paper to discuss the reactions.

Table S2. Comparison between experimental selectivities of reactions A-D and DFT-calculation predicted ones using different basis sets $\mathbf{B 1 - 6}{ }^{\text {a }}$ (solvent effects were computed at the B3LYP level of theory based on the gas-phase optimized structures using the CPCM model and the UA0 radii)

\begin{tabular}{|c|c|c|c|c|c|c|c|c|}
\hline \multirow[t]{2}{*}{ Reaction } & \multirow{2}{*}{\multicolumn{2}{|c|}{$\begin{array}{l}\text { Experimental } \\
\text { Data }\end{array}$}} & \multicolumn{6}{|c|}{$\begin{array}{l}\text { Predicted enantioselectivity and diastereoselectivity } \\
\qquad\left(\Delta \Delta G_{\mathrm{DCM}} \text { in } \mathrm{kcal} / \mathrm{mol}\right)^{\mathrm{b}}\end{array}$} \\
\hline & & & B1 & B2 & B3 & B4 & B5 & B6 \\
\hline A & ee & $94 \%$ & $\begin{array}{c}100 \% \\
(3.8)\end{array}$ & $\begin{array}{c}100 \% \\
(4.5)\end{array}$ & $\begin{array}{l}99 \% \\
(3.3)\end{array}$ & $\begin{array}{c}100 \% \\
(4.5)\end{array}$ & $\begin{array}{l}99 \% \\
(3.2)\end{array}$ & $\begin{array}{c}100 \% \\
(4.3)\end{array}$ \\
\hline \multirow{2}{*}{ B } & ee & $98 \%$ & $\begin{array}{c}100 \% \\
(3.8)\end{array}$ & $\begin{array}{l}100 \% \\
(4.7)\end{array}$ & $\begin{array}{c}100 \% \\
(3.9)\end{array}$ & $\begin{array}{c}100 \% \\
(5.0)\end{array}$ & $\begin{array}{c}100 \% \\
(4.1)\end{array}$ & $\begin{array}{c}100 \% \\
(4.1)\end{array}$ \\
\hline & $\mathrm{dr}$ & $>50: 1$ & $\begin{array}{l}69: 1 \\
(2.5)\end{array}$ & $\begin{array}{l}198: 1 \\
(3.1)\end{array}$ & $\begin{array}{l}86: 1 \\
(2.6)\end{array}$ & $\begin{array}{l}430: 1 \\
(3.6)\end{array}$ & $\begin{array}{l}190: 1 \\
(3.1)\end{array}$ & $\begin{array}{l}25: 1 \\
(1.9)\end{array}$ \\
\hline C & ee & $85 \%$ & $\begin{array}{l}97 \% \\
(2.5) \\
\end{array}$ & $\begin{array}{l}99 \% \\
(3.4)\end{array}$ & $\begin{array}{l}83 \% \\
(1.4) \\
\end{array}$ & $\begin{array}{l}99 \% \\
(3.3) \\
\end{array}$ & $\begin{array}{l}80 \% \\
(1.3) \\
\end{array}$ & $\begin{array}{c}100 \% \\
(3.6) \\
\end{array}$ \\
\hline D & ee & $82 \%$ & $\begin{array}{l}60 \% \\
(0.8)\end{array}$ & $\begin{array}{l}85 \% \\
(1.5)\end{array}$ & $\begin{array}{l}68 \% \\
(1.0)\end{array}$ & $\begin{array}{l}86 \% \\
(1.5)\end{array}$ & $\begin{array}{l}75 \% \\
(1.2)\end{array}$ & $\begin{array}{c}8 \% \\
(0.1)\end{array}$ \\
\hline
\end{tabular}

a B1: the Ahlrichs' SVP all-electron basis set for the zinc atom and the 6-31G(d) basis set for other atoms except for the iodine atom, for which the LANL2DZ basis set was used; B2: the LANL2DZ basis set for zinc and iodine atoms and the 6-31G(d) basis set for the other atoms; B3: the SDD 
basis set for zinc and iodine atoms and the 6-31G(d) basis set for the other atoms; B4: the LANL2DZ basis set for zinc and iodine atoms and the 6-311G(d,p) basis set for the other atoms; B5: the SDD basis set for zinc and iodine atoms and the 6-311G(d,p) basis set for the other atoms; B6: the LANL2DZ basis set for the iodine atom and the 6-311G(d,p) basis set for the other atoms. ${ }^{\text {b }} \Delta \Delta G_{\mathrm{DCM}}$ : the relative energies of two competing enantio-/diastereo-selective cyclopropanation transition states.

Table S3. Comparison between experimental selectivities of reactions A-D and DFT-calculation predicted ones using different basis sets B1-6 ${ }^{\text {a }}$ (solvent effects were computed at the B3LYP level of theory based on the gas-phase optimized structures using the CPCM model and the UAKS radii)

\begin{tabular}{|c|c|c|c|c|c|c|c|c|}
\hline \multirow[t]{2}{*}{ Reaction } & \multirow{2}{*}{\multicolumn{2}{|c|}{$\begin{array}{l}\text { Experimental } \\
\text { Data }\end{array}$}} & \multicolumn{6}{|c|}{$\begin{array}{l}\text { Predicted enantioselectivity and diastereoselectivity } \\
\qquad\left(\Delta \Delta G_{\mathrm{DCM}} \text { in } \mathrm{kcal} / \mathrm{mol}\right)^{\mathrm{b}}\end{array}$} \\
\hline & & & B1 & B2 & B3 & B4 & B5 & B6 \\
\hline $\mathbf{A}$ & ee & $94 \%$ & $\begin{array}{c}100 \% \\
(4.6) \\
\end{array}$ & $\begin{array}{c}100 \% \\
(5.2) \\
\end{array}$ & $\begin{array}{r}100 \% \\
(4.0) \\
\end{array}$ & $\begin{array}{c}100 \% \\
(5.2)\end{array}$ & $\begin{array}{l}100 \% \\
(3.9)\end{array}$ & $\begin{array}{c}100 \% \\
(5.1)\end{array}$ \\
\hline \multirow{2}{*}{ B } & ee & $98 \%$ & $\begin{array}{r}100 \% \\
(4.4) \\
\end{array}$ & $\begin{array}{c}100 \% \\
(5.3) \\
\end{array}$ & $\begin{array}{r}100 \% \\
(4.4) \\
\end{array}$ & $\begin{array}{c}100 \% \\
(5.5)\end{array}$ & $\begin{array}{c}100 \% \\
(4.5) \\
\end{array}$ & $\begin{array}{c}100 \% \\
(4.6) \\
\end{array}$ \\
\hline & $\mathrm{dr}$ & $>50: 1$ & $\begin{array}{r}251: 1 \\
(3.3) \\
\end{array}$ & $\begin{array}{l}706: 1 \\
(3.9) \\
\end{array}$ & $\begin{array}{l}304: 1 \\
(3.4) \\
\end{array}$ & $\begin{array}{c}1344: 1 \\
(4.3)\end{array}$ & $\begin{array}{l}596: 1 \\
(3.8) \\
\end{array}$ & $\begin{array}{l}73: 1 \\
(2.5) \\
\end{array}$ \\
\hline C & ee & $85 \%$ & $\begin{array}{l}99 \% \\
(3.3) \\
\end{array}$ & $\begin{array}{c}100 \% \\
(4.2) \\
\end{array}$ & $\begin{array}{l}94 \% \\
(2.1) \\
\end{array}$ & $\begin{array}{c}100 \% \\
(4.1) \\
\end{array}$ & $\begin{array}{l}93 \% \\
(2.0) \\
\end{array}$ & $\begin{array}{c}100 \% \\
(4.5) \\
\end{array}$ \\
\hline D & ee & $82 \%$ & $\begin{array}{l}65 \% \\
(0.9)\end{array}$ & $\begin{array}{l}87 \% \\
(1.6)\end{array}$ & $\begin{array}{l}71 \% \\
(1.1)\end{array}$ & $\begin{array}{l}88 \% \\
(1.7)\end{array}$ & $\begin{array}{l}79 \% \\
(1.3)\end{array}$ & $\begin{array}{l}21 \% \\
(0.3)\end{array}$ \\
\hline
\end{tabular}

a B1: the Ahlrichs' SVP all-electron basis set for the zinc atom and the 6-31G(d) basis set for other atoms except for the iodine atom, for which the LANL2DZ basis set was used; B2: the LANL2DZ basis set for zinc and iodine atoms and the 6-31G(d) basis set for the other atoms; B3: the SDD basis set for zinc and iodine atoms and the 6-31G(d) basis set for the other atoms; B4: the LANL2DZ basis set for zinc and iodine atoms and the 6-311G(d,p) basis set for the other atoms; B5: the SDD basis set for zinc and iodine atoms and the 6-311G(d,p) basis set for the other atoms; B6: the LANL2DZ basis set for the iodine atom and the 6-311G(d,p) basis set for the other atoms. ${ }^{\text {b }} \Delta \Delta G_{\mathrm{DCM}}$ : the relative energies of two competing enantio-/diastereo-selective cyclopropanation transition states. 
We then conducted the comparison of stability and reactivity between tetramer 2-T and the chiral zinc complex 2-L-O (for details, see Figure 3 in the paper) using different basis sets B1-6 and radii sets (Table S4). It was found that all these calculations give similar results: the chiral zinc complex 2-L-O is much more stable than tetramer 2-T, and the enantioselective cyclopropanation pathway via transition state TS-3a is more favorable than the background reaction (via TS-2-T) leading to racemic products. This further indicates that the choice of different basis sets and radii sets has limited influence on understanding and predicting the reactivities of both asymmetric and racemic Simmons-Smith reactions. As suggested by this reviewer, the Ahlrichs' SVP all-electron basis set would underestimate the reaction barriers for Simmons-Smith type cyclopropanation reactions. The computed energy barriers using previously chosen basis set $\mathbf{B} 1$ are about $3 \mathrm{kcal} / \mathrm{mol}$ lower than those using basis set B5. Further calculations with basis set B5 showed that the background cyclopropanation requires an overall activation free energy of $18.5 \mathrm{kcal} / \mathrm{mol}$ (for details, see Figure 1 in the paper). This result is in good agreement with the experimental observation reported by Charette and co-workers: the (E) $-\mathrm{PhCH}=\mathrm{CHCH}_{2} \mathrm{OZnCH}_{2} \mathrm{I}$ was not stable for a long period of time at $-20{ }^{\circ} \mathrm{C}$, and its corresponding cyclopropane product appeared within $24 \mathrm{~h}$. These computational results also support the choice of basis set $\mathbf{B 5}$ to investigate the reactions in the paper.

Table S4. Comparison of stability and reactivity between tetramer 2-T and the chiral zinc complex 2-L-O using different basis sets B1-6 ${ }^{\text {a }}$ (solvent effects were computed at the B3LYP level of theory based on the gas-phase optimized structures using the CPCM model and the UA0 radii, and the results using the UAKS radii were given in the parenthesis)

\begin{tabular}{|c|c|c|c|c|c|c|}
\hline \multirow{2}{*}{ Structure } & \multicolumn{6}{|c|}{$\Delta G_{\mathrm{DCM}} \mathrm{in} \mathrm{kcal} / \mathrm{mol}$} \\
\cline { 2 - 7 } & $\mathbf{B 1}$ & $\mathbf{B 2}$ & $\mathbf{B 3}$ & $\mathbf{B 4}$ & $\mathbf{B 5}$ & $\mathbf{B 6}$ \\
\hline 2-T & $0.0(0.0)$ & $0.0(0.0)$ & $0.0(0.0)$ & $0.0(0.0)$ & $0.0(0.0)$ & $0.0(0.0)$ \\
\hline TS-2-T & $14.6(15.9)$ & $13.7(14.9)$ & $15.1(16.1)$ & $15.0(16.0)$ & $16.5(17.4)$ & $18.1(19.4)$ \\
\hline 2-L-O & $-5.4(-7.0)$ & $-6.2(-7.8)$ & $-7.2(-8.8)$ & $-3.5(-5.0)$ & $-3.9(-5.4)$ & $-4.2(-5.7)$ \\
\hline TS-3a & $10.3(12.6)$ & $8.6(10.9)$ & $8.8(11.0)$ & $13.3(15.4)$ & $14.4(16.4)$ & $15.3(17.8)$ \\
\hline
\end{tabular}

a B1: the Ahlrichs' SVP all-electron basis set for the zinc atom and the 6-31G(d) basis set for other atoms except for the iodine atom, for which the LANL2DZ basis set was used; B2: the LANL2DZ basis set for zinc and iodine atoms and the 6-31G(d) basis set for the other atoms; B3: the SDD basis set for zinc and iodine atoms and the 6-31G(d) basis set for the other atoms; B4: the LANL2DZ basis set for zinc and iodine atoms and the 6-311G(d,p) basis set for the other atoms; B5: the SDD basis set for zinc and iodine atoms and the 6-311G(d,p) basis set for the other atoms; B6: the LANL2DZ basis set for the iodine atom and the 6-311G(d,p) basis set for the other atoms. 


\section{DFT-Computed Free Energy Surfaces for Reactions A-D}

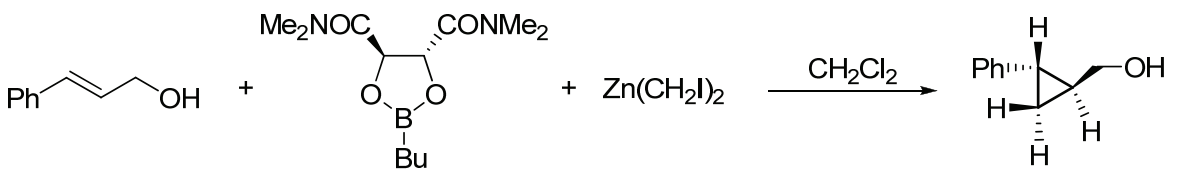

$$
\begin{aligned}
& 2 a \\
& 1
\end{aligned}
$$

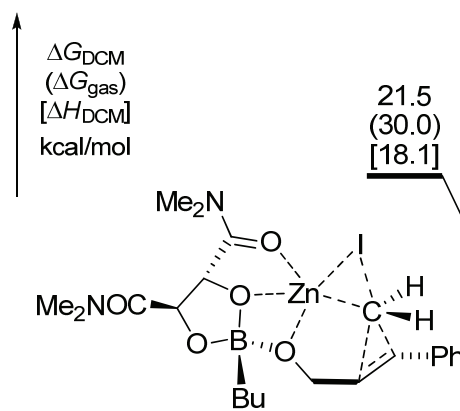

TS-ent-3a

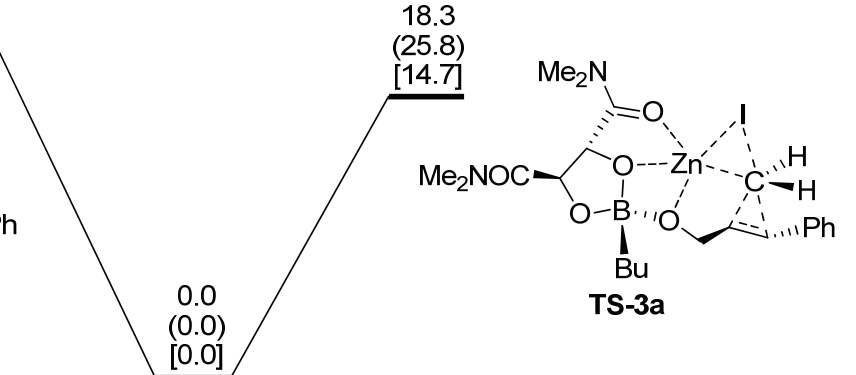

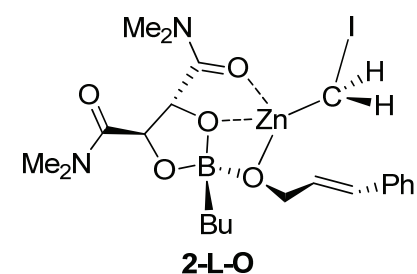

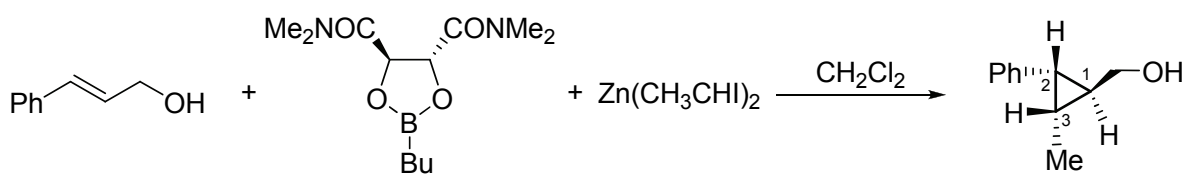
1
$2 a$

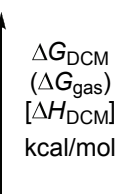

$3 b$

$98 \%$ ee, $>50: 1 \mathrm{dr}$

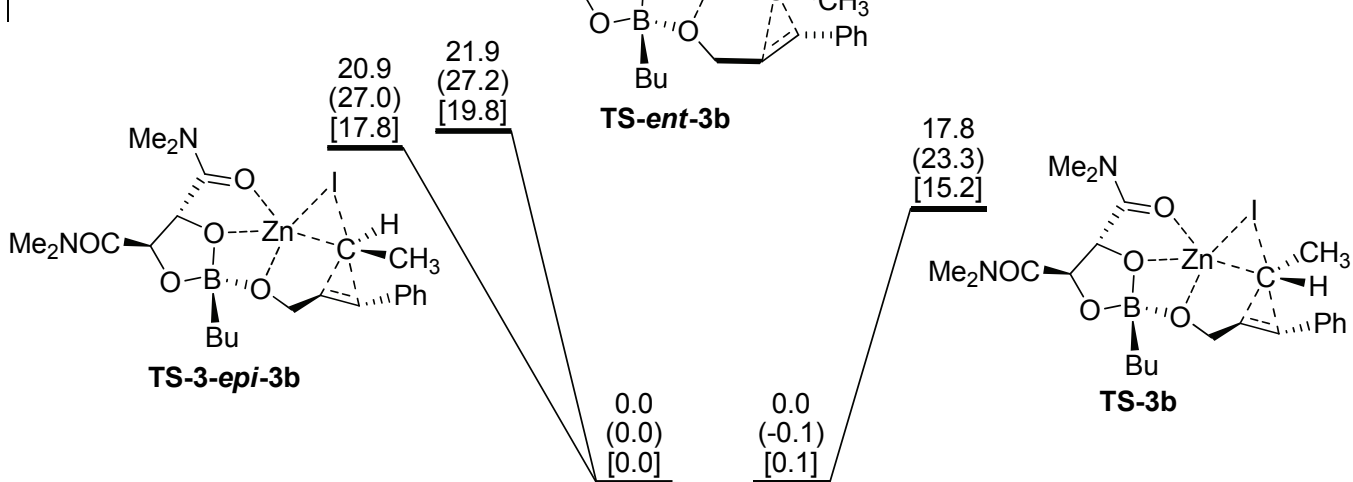

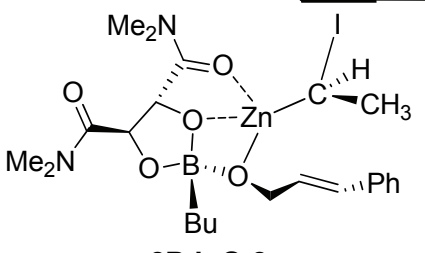

2B-L-O-2

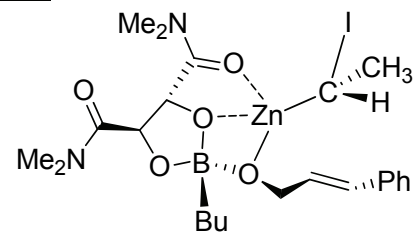

2B-L-O-1 


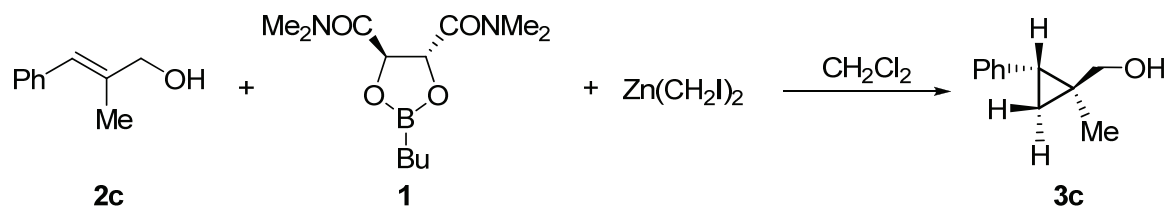

$85 \%$ ee
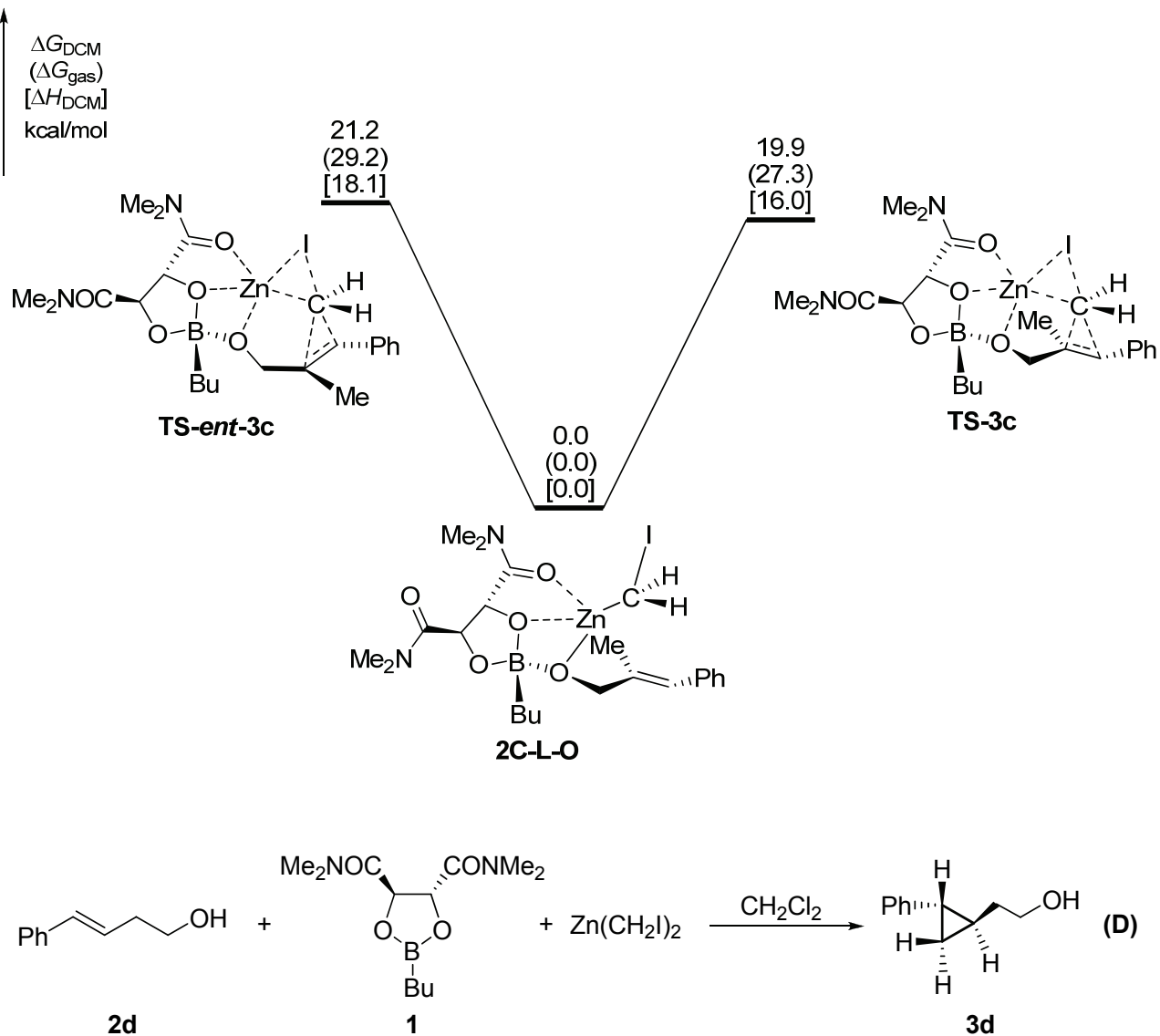

$82 \%$ ee $\Delta G_{\mathrm{DCM}}$
$\left(\Delta G_{\mathrm{gas}}\right)$
$\left[\Delta H_{\mathrm{DCM}}\right]$
$\mathrm{kcal} / \mathrm{mol}$

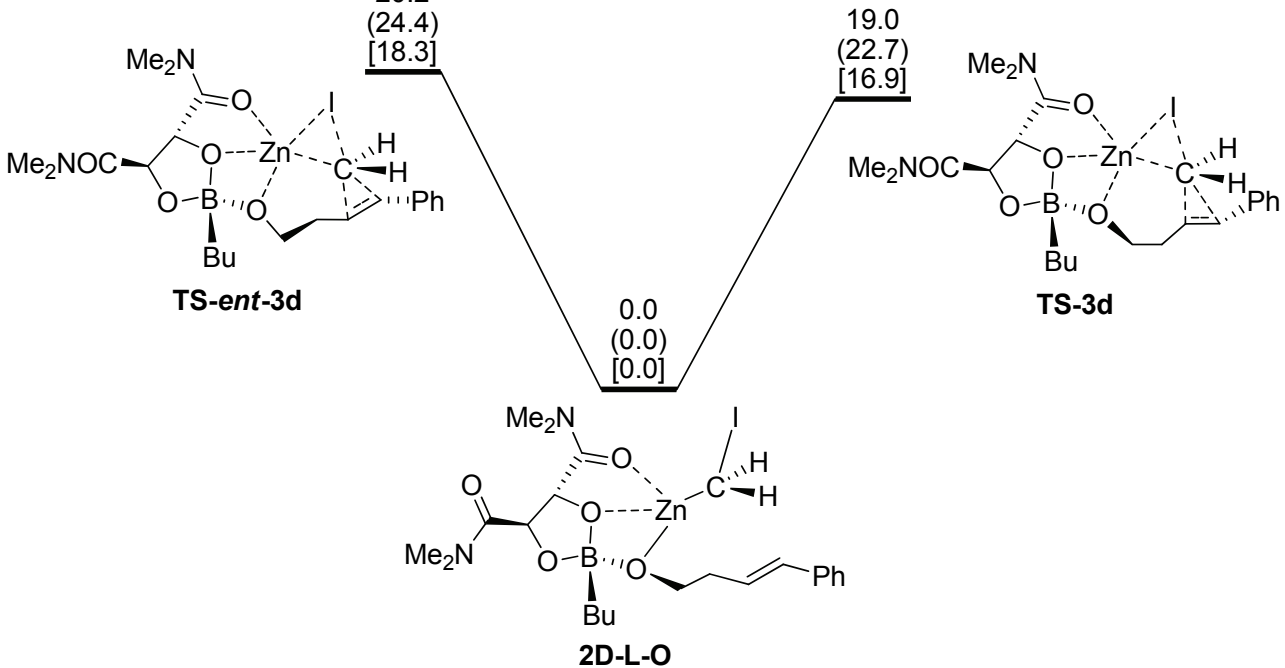

